\title{
2nd Cardiovascular Forum for Promoting Centres of Excellence and Young Investigators
}

\author{
September 4 to 6, 2014 • Winnipeg, Manitoba
}

001

LEFT VENTRICULAR SYSTOLIC FUNCTION IN METASTATIC RENAL CELL CANCER PATIENTS TREATED WITH SUNITINIB OR PAZOPANIB THERAPY: MULTIMODALITY ANALYSIS OF LEFT VENTRICULAR EJECTION FRACTION OVER 6 MONTHS SS Abdelmoneim ${ }^{1}$, LF Nhola ${ }^{1}$, R Huang ${ }^{1}$, M Kohli ${ }^{1}$, S Longenbach ${ }^{1}$, M Green ${ }^{1}$, HR Villarraga ${ }^{1}, \mathrm{KA}$ Bordun ${ }^{2}$, DS Jassal', SL Mulvagh'

${ }^{1}$ Mayo Clinic, Rochester, Minnesota, USA; ${ }^{2}$ University of Manitoba, Winnipeg, Manitoba

We evaluated serial LV ejection fraction (LVEF) as measured by 2-dimensional biplane Simpson's methods (2-DE) and 3-dimensional echocardiography (3-DE) with and without cardiac-ultrasound contrast up to 6 months after tyrosine kinase-inhibitors (TKI) therapy in 12 pts with metastatic renal cell carcinoma (mRCC) (10 males; age $62 \pm 9$ years) at four time points [ 33 echos: baseline, 1,3 and 6 months after TKI therapy]. Repeatmeasure analyses were used to compare LVEF across imav.ging modalities and over time. LVEF remained unchanged across all 4 imaging modalities. LVEF showed a non-significant reduction from baseline to 6 months (mean EF difference (SE) for 2DE-unenhanced, 3DE-unenhanced, 2DE-enhanced and 3DE-enhanced $[-2.2(2.9), \mathrm{P}=0.49 ;-2.9(3.6), \mathrm{P}=0.48 ;-2.2(0.78)$, $\mathrm{P}=0.07$; and $-1.9(2.3), \mathrm{P}=0.46$, respectively]. Conclusions: No significant temporal changes were found for echo monitored LVEF despite a trend for reduction during 6 months of TKI therapy, suggesting a noncardiotoxic profile.

Funding: ASPER Foundation.

\section{2}

\section{VITAMIN C PROVIDES CARDIOPROTECTION AGAINST} DOXORUBICIN-INDUCED NITROSATIVE AND OXIDATIVE STRESS

G Akolkar, AK Bagchi, S Mandal, DS Jassal, PK Singal

Institute of Cardiovascular Sciences, University of Manitoba,

Winnipeg, Manitoba

Increased oxidative stress (OS) resulting from increased production of reactive oxygen species and reduction in antioxidant reserve is associated with doxorubicin (Dox)-induced cardiotoxicity. High concentration of nitric oxide (NO) by induction of iNOS and peroxynitrite formation may be involved in this cardiotoxicity. We have reported that supplementation of Vitamin C (Vit C) improved antioxidant status and survival whereas it reduced OS and apoptosis in isolated cardiomyocytes. Present study using isolated cardiomyocytes, investigated the role of Vit $\mathrm{C}$ in Dox-induced nitrosative stress and OS. We show that Dox increased levels of intracellular as well as released NO and increased NOS activity. Protein expression of iNOS was upregulated whereas eNOS expression was downregulated. In contrast, treatment with Vit $\mathrm{C}$ upregulated eNOS and it reduced the expression of iNOS. The posttranslational upregulation of s-nitrosylated proteins by Dox was also mitigated by Vit $\mathrm{C}$. These data suggest that cardioprotective effect of Vit $\mathrm{C}$ treatment may be mediated by a reduction in nitrosative and OS induced by Dox.

Funding: Canadian Institutes of Health Research and Alfred Deacon Foundation.
003

STEM CELL THERAPY PREVENTED DOXORUBICIN INDUCED CARDIAC DYSFUNCTION IN DIABETIC RATS

HI Ammar ${ }^{1}$, E Abu-El Rub ${ }^{2}$, MB Nashed ${ }^{1}$, RI Ammar', HM Gabr $^{1}$, HE Elsayed ${ }^{1}$, S Dhingra ${ }^{2}$

${ }^{1}$ Department of Physiology, Cairo University, Egypt; ${ }^{2}$ St Boniface Research Centre, University of Manitoba, Winnipeg, Manitoba Doxorubicin (DOX) is a well known anticancer drug; however, its clinical use was limited due to cardiotoxic effects. One of the major concerns with DOX therapy is its toxicity in patients who are frail, particularly diabetics. Several studies suggested that mesenchymal stem cell (MSC) therapy has potential to restore cardiac function after DOX induced injury. However, limited data is available on the effects of MSC therapy on DOX induced cardiac dysfunction in diabetics and it was done in current study. Diabetes was induced in rats by streptozotcin injection (STZ, $65 \mathrm{mg} / \mathrm{kg}$ b.w, i.p.). After 4 weeks, glucose levels in STZ group were significantly greater than control group. Diabetic rats were treated with DOX (adriamycin, $2.5 \mathrm{mg} / \mathrm{kg}$ b.w, i.p) 3 times/week for 2 weeks (AD group); or with $\mathrm{AD}+$ bone marrow MSCs (BM-MSC, $2 \times 10^{6}$ cells, i.v.) or with AD+adipose tissue MSCs (AD-MSC $2 \times 10^{6}$ cells). After 4 weeks, AD treatment significantly decreased \%FS, LVDP, dp/dt max and rate pressure product (RPP). Both BM-MSC and AD-MSC prevented DOX induced decrease in these parameters. In conclusion BM-MSC and AT-MSC were equally effective in preserving cardiac function after DOX therapy in diabetic rats.

004

CAMKII OXIDATIVE ACTIVATION AND ITS INFLUENCE ON PATHOGENESIS OF ISCHEMIC/REPERFUSED HEART: THE ROLE OF AT1 RECEPTORS

T Rajtik ${ }^{1}$, A Szobi ${ }^{1}$, S Carnicka ${ }^{2}$, Z Giricz ${ }^{3}$, P Svec ${ }^{1}$, P Ferdinandy $^{3}$, T Ravingerova ${ }^{2}$, A Adameova ${ }^{1}$

${ }^{1}$ Department of Pharmacology and Toxicology, Faculty of Pharmacy, Comenius University; ${ }^{2}$ Institute for Heart Research, Slovak Academy of Sciences, Bratislava, Slovak Republic; ${ }^{3}$ Department of Pharmacology and Pharmacotherapy, Faculty of Medicine, Semmelweis University, Budapest, Hungary

$\mathrm{Ca}^{2+} /$ calmodulin-dependent protein kinase II (CaMKII) is a crucial regulator of excitation-contraction coupling (ECC) in the heart and its activation by $\mathrm{Ca}^{2+}$-overload and excessive reactive oxygen species (ROS) promotes signaling pathways underlying heart dysfunction. Of all sources of ROS, NADPH (NOX) oxidase system through the stimulation of the AT1 receptors seems to play a very important role in the CaMKII oxidative activation. Thus, in heart diseases characterized by elevated levels of angiotensin II, including ischemia/reperfusion (I/R) injury, the alterations in the AT1NOX-CaMKII signaling may be responsible for disturbances in ECC.

We will highlight the role of oxidative stress in the CaMKII activation and discuss if the inhibition of the AT1 receptors may reduce the incidence of $\mathrm{I} / \mathrm{R}$-induced arrhythmias and mitigate contractile dysfunction due to influence on CaMKII.

Acknowledgements: VEGA 1/0638/12, 2/0054/11, APVV 0102-11

\section{5}

\section{EPICARDIAL ADIPOSE TISSUE, OBESITY AND} INFLAMMATION IN ATHEROSCLEROSIS

D Agrawal, V Swier, L Tang, C Pelham, M Radwan, M Del Core, W Hunter

Creighton University School of Medicine, Omaha, Nebraska, USA

Patients with obesity and insulin resistance develop accelerated atherosclerosis and pro-inflammatory changes in epicardial adipose tissue (EAT) 
could exaggerate intimal hyperplasia following coronary intervention. We examined if obesity-induced inflammatory phenotype of EAT is exacerbated by vitamin $\mathrm{D}$ deficiency leading to endothelial dysfunction and enhanced intimal hyperplasia. Atherosclerotic lesions in Yucatan microswine fed with high-fructose high cholesterol diet were confirmed by coronary angiography and histology. Immunostaining was performed in the adipose tissue and coronary arteries for macrophage phenotype. We found significantly increased infiltration of CD86+ cells (M1 mФ), dense infiltratory cells with marked increase in TNF- $\alpha$, MCP-1, IL-6 expression with minimal immunostaining to CD206 (M2 $\mathrm{m} \Phi)$ in adipocytes and coronary arteries compared to vitD-deficient or vitD-sufficient swine EAT. High contractility to serotonin and attenuated dilation was observed in coronary arteries of vitD-deficient atherosclerotic swine than in vitD-sufficient group. Thus, high-fructose high-cholesterol diet enhances pro-inflammatory macrophages, increases vasoconstriction and decrease vasodilatation. Vitamin D supplementation could be beneficial in preventing metabolic effects in atherosclerosis.

\section{6}

NATRIURETIC PEPTIDE RECEPTOR-C, GI PROTEINS AND REGULATION OF BLOOD PRESSURE

\section{MB Anand-Srivastava}

Department of Molecular and Integrative Physiology, University of Montreal, Montreal, Quebec

An increased expression of inhibitory $G$ proteins (Gi) was shown as a contributing factor in the pathogenesis of hypertension in spontaneously hypertensive rats (SHR). C-ANP ${ }_{4-23}$, that specifically interacts with natriuretic peptide receptor- $\mathrm{C}$ (NPR-C) was shown to decrease the enhanced expression of Gia proteins in vascular smooth muscle cells from SHR. In the present study, we investigated if in vivo treatment of SHR with C-ANP ${ }_{4-23}$ could attenuate the development of high blood pressure (BP) and explore the underlying mechanism/s. The BP started increasing in SHR at 4 weeks and increased to about $190 \mathrm{mmHg}$ at 8 weeks. However, intraperitoneal injection of $\mathrm{C}-\mathrm{ANP}_{4-23}$ to prehypertensive SHR attenuated the development of high BP and was decreased by about $50 \mathrm{mmHg}$ at 8 weeks. The C-ANP ${ }_{4-23}$ treatment also decreased the enhanced levels of Gia proteins, superoxide anion, peroxynitrite, NADPH oxidase activity and the enhanced expression of NOX-4, P47phox, nitrotyrosine, and decreased levels of eNOS in hearts and aorta without affecting the levels of NPR-A/ NPR-C. These results indicate that NPR-C activation by C-ANP $\mathrm{A}_{4-23}$ attenuates the development of high BP in SHR through the inhibition of enhanced levels of Gia proteins and nitroxidative stress and not through eNOS /cGMP pathway.

Funding: Supported by CIHR.

\section{7}

\section{CHANGES IN PATIENT FOOD CHOICES OVER 1 YEAR IN THE FLAXPAD TRIAL}

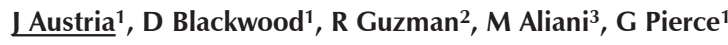

${ }^{1}$ Department of Physiology, CCARM, St Boniface General Hospital;

2 Department of Surgery ACRI, St Boniface General Hospital;

${ }^{3}$ Department of Human Nutritional Sciences, University of Manitoba, Winnipeg, Manitoba

Functional foods in nutritional studies require foods to be palatable in order that patients are adherent. The FlaxPAD study is a double-blinded, randomized, placebo-controlled study. Patients (110) with peripheral arterial disease (PAD) were randomized to consume food products containing $30 \mathrm{~g}$ of flaxseed (FX) or placebo (PL) per day. Products include sprinkles, pasta, snack bars, biscuits, buns, bagels and muffins. They ranked the products from most to least preferred. Monthly rations were delivered and recorded according to the patients' choice over the 1 -year. After 6 months, the drop-out rate was $22.4 \%$ and $21.2 \%$ for the FX and PL groups, respectively $(\mathrm{P}>0.05)$. Feedback and monthly ration show that bagels and muffins were the most preferred. The cinnamon raisin bagels were most liked. Pasta was least desired. Diversity of food products from which the patients could choose over the course of the trial was critical to maintaining compliance. Further research to develop other palatable products would enhance compliance in the future.
Acknowledgements: Flax2015, Canola Council of Canada, ARDI, CIHR, CIGI, FDC Canada Bread, Glanbia Nutritionals Inc, St Boniface Hospital Foundation.

\section{8}

\section{THE IMPORTANCE OF AN INTEGRATIVE APPROACH TO CARDIOVASCULAR PREVENTION AND HEALING}

\section{C) Baechler}

Allina Health-Penny George Institute for Health and Healing,

Minneapolis, Minnesota, USA

As western cardiology has become more advanced in technology, interventions, and medical therapies, the emphasis of the heart in isolation has been emphasized. Integrative cardiology is defined as healing-oriented medicine that takes account the whole person (mind, body, and spirit) as well as all aspects of lifestyle; it emphasizes the therapeutic relationship and makes use of appropriate therapies, both western and eastern. Heart disease is largely preventable, but a successful approach necessitates a holistic approach. This evidence base for integrative cardiology approaches will be explored. Areas that will be highlighted include: nutrition and cardiovascular health, the impact and type of exercise with most promising results, botanical medicine and cardiovascular disease, when/where/how to use acupuncture in the cardiovascular patient, spirituality and heart health, mind-body approaches to heart health, clinical applications to hypertension, coronary disease, and heart failure. $74 \%$ of all Americans report using some sort of integrative health. There is a growing importance for clinicians to have knowledge of the evidence based practices as well as acceptance for patients to have optimal outcomes.

\section{9}

\section{IL-10 IMPROVES CARDIOMYOCYTE SURVIVAL THROUGH IL-1B-MEDIATED MYD88-DEPENDENT TLR4 INNATE SIGNALING}

AK Bagchi ${ }^{1}{ }^{2}$, G Akolkar, X Gao ${ }^{2}$, X Yang ${ }^{2}$, PK Singal ${ }^{1,2}$

${ }^{1}$ Institute of Cardiovascular Sciences, St Boniface Research Centre; ${ }^{2}$ University of Manitoba, Winnipeg, Manitoba

IL-10 is known to play a cardioprotective role in suppressing the effects of TNF- $\alpha$-induced oxidative damage via ERK1/2/Jak/Stat3. We have already reported that IL-10-mediated cardiac cell survival is regulated through its innate signaling via the activation of toll-like receptor 4 (TLR4) and is dependent on myeloid differentiation gene factor 88 (MyD88). In this study, we examined the role of Jak/Stat 3 in IL-10-induced activation of TLR4. We are now reporting that Stat 3 inhibition attenuates IL-10induced activation of TLR4, suggesting that TLR4 stimulation is downstream to $\mathrm{Akt}^{\mathrm{ser} 86} / \mathrm{Jak} / \mathrm{Stat} 3$ activation. Further downstream, there was an upregulation of the synthesis of IL-1 mRNA. In addition, we also show that IL-10 helps in the dissociation of interleukin-1 receptor activated kinase 4 (IRAK4) into IRAK1 instead of IRAK2 and there was downregulation of TNF- $\alpha$ receptor associated inducible protein (TRAIP). These data suggest that recruitment of MyD88/IRAK1/IRAK4, blocks TNF- $\alpha$ R associated changes in the cell. Upregulation of TLR4 signaling by IL-10 involving Akt-Jak/Stat3 pathway could be a better therapeutic strategy in cardiovascular diseases. Acknowledgments: Funding provided by Canadian Institute of Health Research to PKS.

\section{0}

SCLERAXIS IS A CRITICAL REGULATOR OF CARDIAC EXTRACELLULAR MATRIX SYNTHESIS

RA Bagchi ${ }^{1}$, R Schweitzer ${ }^{2}$, MP Czubryt ${ }^{1}$

${ }^{1}$ Institute of Cardiovascular Sciences, University of Manitoba, Winnipeg, Manitoba; ${ }^{2}$ Oregon Health and Science University, Portland, Oregon, USA

Our laboratory has previously reported the required role of the transcription factor Scleraxis $(\mathrm{Scx})$ in regulation of human collagen 1a2 (Col1a2) gene expression - a predominant component of cardiac extracellular matrix. Here we elucidate the cross-talk between Scx and Smads via the pro-fibrotic TGF $\beta$ signaling pathway in regulation of Colla2 expression. Gene reporter assays, qPCR and immunoblotting demonstrate that Scx and Smads synergistically co-regulate collagen gene expression and interference with Scx 
function abrogates collagen gene expression effected by TGF $\beta$. Congenital absence of Scx results in dramatic reduction of myocardial fibrillar collagens, proteoglycans and matrix-degrading enzymes (MMPs) as demonstrated by histology, qPCR, immunoblotting and gelatin zymography. Many of these genes were up-regulated in response to Scx over-expression, suggesting a direct action of Scx on gene transcription. A significant reduction in $\mathrm{DDR}^{2+}$ fibroblasts in Scx null hearts contributed to these matrix-associated changes. Thus Scx critically regulates the composition of the cardiac extracellular matrix through its effect on fibroblast population in the heart. Acknowledgements: Grant funding provided by CIHR to MPC.

\section{1}

\section{IMPACT OF MEDICATION ADHERENCE ON LONG-TERM} OUTCOMES POST MYOCARDIAL INFARCTION

$\underline{\mathrm{S} \text { Bansilal }{ }^{1} \text {, J Castellano }}{ }^{2}$, $\mathrm{H} \mathrm{Wei}^{3}$, E Vinado ${ }^{4}$, A Freeman ${ }^{5}$,

C Spettell $^{5}$, F Alonso ${ }^{4}$, G Steinberg ${ }^{3}$, G Sanz ${ }^{2}$, V Fuster ${ }^{1}$

${ }^{1}$ Mount Sinai Hospital, New York, New York, USA; ${ }^{2}$ CNIC, Madrid, Spain; ${ }^{3}$ Aetna Inc, New York, USA; ${ }^{4}$ Grupo Ferrer, Barcelona, Spain; ${ }^{5}$ Aetna Inc, Blue Bell, Pennsylvania, USA

BACKGROUND: We attempted to study the association between levels of medication adherence and long term MACE in patients post MI.

METHODS: We queried a U.S.-based health insurers' claims data for patients hospitalized for MI between $1 / 1 / 10$ and 2/28/13. The primary outcome measure was a composite of all-cause death, MI, Stroke or coronary revascularization (MACE). Using proportion of days covered (PDC), patients were stratified as fully adherent ( $\mathrm{FA} \geq 80 \%)$, partially adherent (PA $\geq 40 \%$ to $\leq 79 \%$ ) or non-adherent (NA $<40 \%$ ).

RESULTS: Of the 4015 patients, 1031 (26\%) were classified as NA, $1263(31 \%)$ as PA and $1721(43 \%)$ as FA. At 2 yrs follow up, the FA group had significantly lower rate of MACE than the NA $[18.9 \%$ vs. $26.3 \%$; $0.73(0.61-0.86), \mathrm{P}<0.0004]$ and the $\mathrm{PA}$ groups $[18.9 \%$ vs. $24.7 \%$; $0.81(0.69-0.96), P<0.016]$. No statistical difference was observed between the NA and PA groups $(\mathrm{P}=0.22)$.

CONCLUSIONS: Being fully adherent was associated with a lower rate of MACE compared to partial or non-adherence. A polypill enabling $>80 \%$ adherence may significantly reduce CV events post MI.

\section{2}

\section{INTERVENTRICULAR DIFFERENCES IN CARDIOMYOCYTE CONTRACTILITY IN A RAT MODEL OF POST-ISCHEMIC HEART FAILURE}

¿ Barta ${ }^{1}$, A Kovacs ${ }^{1}$, SK Sanganalmath ${ }^{2}$, NS Dhalla ${ }^{2}$

${ }^{1}$ University of Debrecen, Institute of Cardiology, Debrecen,

Hungary; ${ }^{2}$ St Boniface Hospital Research Centre, Institute of Cardiovascular Sciences, Winnipeg, Manitoba

Right ventricular (RV) remodeling in left ventricular (LV) infarctioninduced heart failure (HF) was studied in a rat model. $\mathrm{Ca}^{2+}$-activated force $\left(\mathrm{F}_{\max }\right), \mathrm{Ca}^{2+}$-sensitivity $\left(\mathrm{pCa}_{50}\right)$ and passive force $\left(\mathrm{F}_{\text {pass }}\right)$ were measured in isolated cardiomyocytes. Protein phosphorylation $(\mathrm{P})$ was analyzed. The effect of in vivo treatment with cilostazol (CIL) or sarpogrelate (SAR) was also tested in the RV. $\mathrm{F}_{\max }$ and $\mathrm{pCa}_{50}$ of $\mathrm{RV}$ cells were similar in the HF, Sham, SAR and CIL treated groups. $F_{\text {pass }}$ of RV cells in HF was lower compared to Sham. CIL did not, only SAR increased $\mathrm{F}_{\text {pass }}$. LV cells had higher $\mathrm{F}_{\max }$ and $\mathrm{pCa}_{50}$ in HF. LV $\mathrm{F}_{\text {pass }}$ was unaltered in HF. In HF, total $\mathrm{P}$ of titin and myosin binding protein $\mathrm{C}$ (MyBP-C) was similar in the two ventricles, but $\mathrm{P}$ at Ser-282 of MyBP-C was decreased in the RV. In HF both the RV and the LV Troponin I (TnI) P was lower than in the Sham. In the RV CIL decreased titin P as well as overall and Ser-282 P of MyBP-C, while SAR increased total and Ser-22/23 P of TnI. We found distinct changes in RV cardiomyocytes in post-ischemic HF. Reduced $\mathrm{RV} \mathrm{F}_{\text {pass }}$ might be due to hypoP of PKA sites of MyBP-C. CIL and SAR have different potentials to induce reverse remodeling in the RV.

\section{3}

\section{EFFECTS OF QUERCETIN ON HEARTS ISOLATED FROM NORMAL AND DOXORUBICIN-TREATED RATS}

\section{Bartekova, M Ivanova, P Simoncikova, L Okruhlicova}

M Barancik

Institute for Heart Research, SAS, Bratislava, Slovakia

Quercetin (QCT) exerts beneficial effects on various cells, including cardiomyocytes. Although QCT play a role in protecting myocardium from ischemia, its protective mechanisms remain unclear. Present study was conducted to examine effects of QCT on chronic effect of doxorubicin (DOX) and to investigate mechanisms underlying these effects. Rats were treated with DOX ( $3 \mathrm{w}$, cumul. dose $15 \mathrm{mg} / \mathrm{kg}$ ) and with QCT ( $3 \mathrm{w}$ with DOX $+3 \mathrm{w}$ after DOX, $20 \mathrm{mg} / \mathrm{kg} / \mathrm{d})$. The following parameters were deternined: blood pressure (BP, pletyzmography), ischemic tolerance (Langendorff perfusion), protein levels (Western blot), ultrastructural changes (electron microscopy). We found that QCT improved DOXinduced elevation of BP. QCT also improved post-ischemic recovery of hearts (both normal and DOX-treated). Positive effects of QCT on ischemic tolerance were connected with activation of Akt1 followed by GSK-3 $\beta$ down-regulation. Effects of QCT included also elevation of beta-catenin. We can conclude that QCT improved postischemic recovery of hearts from both normal and DOX-treated rats. The data point to important role of Akt kinase pathway in realisation of QCT effects. Moreover, wnt $/ \beta$-catenin pathway may play a role in effects of QCT.

Funding: VEGA SR 2/0140/12, 2/0169/12 and APVV-0348-12.

\section{4}

TARGETED GLYOXALASE-1 GENE TRANSFER TO PREVENTS END-ORGAN COMPLICATIONS IN DIABETES MELLITUS

\section{KR Bidasee ${ }^{1}$, WG Mayhan, J Singh, F Alomar}

${ }^{1}$ Department of Pharmacology and Experimarntal Neurology, University of Nebraska Medical Center, Omaha, Nebraska, USA In spite of an armamentarium of glucose-lowering and monitoring devices, food management, exercise strategies and education initiatives, individuals with diabetes mellitus (DM) continue to develop end-organ complications at rates 3-4 times higher than the general population. To date, the underlying cause for this remain poorly defined, but clinical and laboratory studies suggest that they may be due in part to dysregulation of the endothelial cells (EC) of the microvasculature by methylglyoxal (MG). Because MG regulates key cellular and physiologic functions, globally lowering its level to minimize endothelial dysfunction in DM will precipitate undesirable side effects. In this study using rodent models of DM we identify smooth muscle cells (SMC) of arterioles as a source of 'endothelial-damaging' MG. We also show that lowering MG levels in SMC by increasing expression of the MG-degrading enzyme glyoxalase-1 prevents endothelial dysfunction, vascular leakage, heart failure, kidney failure and cerebral infarct damage following ischemia-reperfusion with minimal side effects. These data suggest that lowering MG in SMC may be an important therapeutic strategy for preventing end-organ damage in DM.

Funding: NIH, GTRP and Nebraska Redox Center.

\section{5}

\section{HEREDITARY CARDIOMYOPATHY: WHO COMES FIRST,} HEART FAILURE OR VASCULAR FAILURE?

\section{$\underline{\text { G Bkaily }}$}

Department of Anatomy and Cell Biology, Faculty of Medicine, University of Sherbrooke, Sherbrooke, Quebec

Hereditary cardiomyopathy is defined as a heart disease. However, in hereditary cardiomyopathy of the hamster ( $\mathrm{HCMHs})$, there is a progressive and significant decrease in mean arterial pressure (MAP) in aging HCMHs in the absence of structural changes in the aortic tissue. In $\mathrm{HCMHs}$, there is an increase in NHE-1 density at the level of plasma membrane and the nucleus (including nuclear envelop membranes) of both vascular endothelial (VECs) and vascular smooth muscle cells (VSMCs). Intracellular measurements showed the presence of cytosolic and nuclear $\mathrm{Na}^{+}$and $\mathrm{Ca}^{2+}$ overloads in VECs and VSMCs from HCMHs. Furthermore, in VECs from HCMH aortas, there is no change in $\mathrm{ET}_{\mathrm{A}} \mathrm{R}$ density at the level of the cytosol but there is a decrease at the level of the nucleus. Knockdown of NHE-1 in 
VSMCs of $\mathrm{HCMH}$ s rescued the cells from $\mathrm{Na}^{+}$overload. Treatment with the NHE-1 inhibitor also prevented heart and vascular failure. In conclusion, hereditary cardiomyopathy is not only a heart disease. Our results also highlight the role of NHE-1 in the development of hypotension and the therapeutic potential of NHE-1 inhibitors in the treatment of the both cardiac and vascular dysfunctions during the development of hereditary cardiomyopathy.

Funding: Supported by the CIHR.

\section{6}

CORRELATION BETWEEN DIASTOLIC VENTRICULAR DYSFUNCTION AND EKG QT DISPERSION

\section{AO Blackman, J Sobral, OM Gomes}

Cardiovascular Foundation Sao Francisco de Assis, ServCor, Belo Horizonte, Brazil

BACKGROUND: In the earlier stages of diastolic dysfunction patients have no symptoms, and identifying patients with asymptomatic dysfunction may give the opportunity to put the progression to heart failure off. QT dispersion is the difference between the longest and shortest QT intervals on the 12-lead ECG and is an estimation of abnormality of repolarization. Identifying patients with asymptomatic diastolic dysfunction may prevent or even postpone the evolution of this disease.

OBJECTIVE: To evaluate the correlation between grade I diastolic dysfunction and EKG QT dispersion.

METHODS: Transversal study, outpatients, age 40-65 years, both genders, asymptomatic, transthoracic echocardiography. Transmitral flow velocity and tissue Doppler imaging mitral annular velocity were analysed to identify grade I diastolic dysfunction (E/A and em/am ratio $<0.8 \mathrm{~m} / \mathrm{s}$ - and E-wave prolonged. A-wave increased and ejection fraction $>55 \%$ ). Patients were then submitted to 12 channel Holter study for measurement of QT dispersion (normal value $<65 \mathrm{~ms}$ ). A normal comparative group was included.

RESULTS: QTd was increased in patients with DDVE grade I $(\mathrm{P}<0.05)$. CONCLUSION: Patients with DDVE grade I had increased QTd, 10,5\% had Qtd $>80$ ms suggesting greater heterogeneity of repolarization.

\section{7}

\section{THE UTILITY OF CARDIAC BIOMARKERS, TISSUE VELOCITY, AND STRAIN RATE IMAGING FOR THE EARLY DETECTION OF BEVACIZUMAB AND SUNITINIB MEDIATED CARDIOTOXICITY}

KA Bordun ${ }^{1}$, S Premecz' ${ }^{1}$ M daSilva ${ }^{1}$, C White ${ }^{1}$, A Muller ${ }^{1}$, Y Li$^{1}$, H Villaraga ${ }^{2}$, J Herrmann², N Sandhu², A Grothey ${ }^{2}$, M Kohli ${ }^{2}$, J Thliveris ${ }^{1}$, M Pitz ${ }^{3}$, PK Singal ${ }^{1}$, S Mulvagh ${ }^{2}$, DS Jassal11,3 ${ }^{1}$ Institute of Cardiovascular Sciences, St Boniface Research Centre, Winnipeg, Manitoba; ${ }^{2}$ Mayo Clinic, Rochester, Minnesota, USA; ${ }^{3}$ Section of Cardiology, Department of Internal Medicine, University of Manitoba, Winnipeg, Manitoba

INTRODUCTION: Novel targeted drugs including Bevaciazumab (BVZ) and Sunitinib (SNT) are associated with cardiotoxicity. Early indices of left ventricular (LV) systolic dysfunction would be useful to address the cardiac safety of these drugs.

OBJECTIVE: Whether cardiac biomarkers, tissue velocity imaging (TVI), and/or strain rate (SR) can detect early cardiac dysfunction.

METHODS: 75 C57Bl/6 mice received one of three drugs: i) $0.9 \%$ saline; ii) BVZ; or iii) SNT and followed for 14 days. Serial blood pressure, high sensitivity troponin I (hsTnI), and echocardiography were performed. Results: BVZ- and SNT-treated mice demonstrated an increase in mean arterial blood pressure (MAP) and hsTnI. TVI and SR values confirmed early LV systolic dysfunction.

CONCLUSIONS: Novel echo techniques can detect early LV systolic dysfunction in a model of drug-mediated cardiomyopathy.

Acknowledgments: St Boniface Hospital Research Foundation.

\section{8}

ROLE OF EXERCISE AND ENERGY DEFICIT ON AGEASSOCIATED IMPAIRMENTS AND BODY COMPOSITION IN OLDER ADULTS WITH CORONARY ARTERY DISEASE (EXODUS TRIAL)

JA Brenes-Salazar, M Singh

Division of Cardiovascular Diseases, Mayo Clinic, Rochester, Minnesota, USA

Small, supervised studies investigating weight loss in healthy volunteers have shown improved physical functioning, gait, strength, metabolic rate and body composition. The broad objective of this pioneer trial is to determine in a prospective, randomized fashion, the influence of energy deficit (ED) and/or exercise on frailty, quality of life (QOL), and body composition in older, obese adults with central obesity and CAD. The primary hypothesis is that among participants in the cardiac rehabilitation, ED along with regular exercise has a positive influence on frailty, QOL and body composition as compared with usual cardiac rehabilitation. In a prospective, open-labeled, randomized clinical trial design, 80 obese/overweight patients, 60 years or older with $\mathrm{CAD}$ and central obesity, who underwent recent coronary revascularization with either PCI or CABG, will be randomized at cardiac rehabilitation to standardized exercise counseling, or supervised exercise training in an ED program to test whether ED with exercise will improve age-associated impairments (AAI), defined as frailty and poor QOL. ED arm participants will aim for $1200 \mathrm{Kcal} / \mathrm{d}$ for women and $1600 \mathrm{~K} \mathrm{cal} / \mathrm{d}$ for men for 24 weeks.

\section{9}

\section{MLIP, A NOVEL MEDIATOR OF CARDIAC HOMEOSTASIS THROUGH MODULATION OF P53 ACTIVITY}

ME Cattin ${ }^{1}$, PG Burgon ${ }^{1,2}$

${ }^{1}$ University of Ottawa Heart Institute; ${ }^{2}$ Departments of Medicine

(Cardiology) \& Cellular and Molecular Medicine, Faculty of

Medicine, University of Ottawa, Ottawa, Ontario

Muscle-enriched A-type lamin interacting protein (MLIP) is ubiquitously expressed, with highest levels in the heart. Its biological function remains elusive but it may be of high relevance for cardiac function, as it has no paralogous homologue suggesting no functional redundancy.

To elucidate MLIP's function, we generated homozygous (MLIP-/-) null mice. By 8 weeks of age, they develop dilated cardiomyopathy. Several lines of evidence indicate that this cardiomyopathy may result from metabolic defects of the myocardium in the absence of MLIP: i) Cardiac gene expression profiling revealed an activation of three metabolic stressinduced pathways involved in tissue homeostasis and regulation of mitochondrial function (FoxO1/3a, STAT3 \& p53 signaling pathways); ii) Oxidation-reduction processes predicted to be down-regulated; iii) cardiac FDG-uptake was decreased despite normal blood glucose level; and iv) preliminary electron microscope observations reveal a possible alteration in mitochondria network. Altogether, these data point toward MLIP's critical role in the maintenance of cardiac homeostasis through p53 mediated regulation of cardiac metabolism and function.

\section{0}

\section{PERSPECTIVES ON THE CARDIOVASCULAR BENEFITS OF BIOACTIVE DIETARY INGREDIENTS AND LIFESTYLE MODIFICATIONS}

\section{H Buttar}

Department of Pathology \& Laboratory Medicine, Faculty of Medicine, University of Ottawa, Ottawa, Ontario

Cardiovascular diseases (CVDs) are the leading cause of death and disability worldwide. Conventional risk factors of CVD are hypertension, atherosclerosis, hyperlipidemia and hyperglycemia. Lifestyle factors such as tobacco use, lack of exercise, unhealthy dietary habits, and low socioeconomic status contribute heavily to the development of obesity, diabetes and CVD in children and adults. Diets rich in whole grains, fruits/vegetables, olive oil, fish, low-fat dairy products, and moderate wine consumption are linked with lower incidence of CVD. Lifestyle changes such as regular physical activity (30 min/day), restriction of caloric and sodium intake, smoking cessation and moderate alcohol consumption help in improving 
heart health and quality of life. Intake of foods containing flavonoids/ antioxidants, vitamins, minerals, and amino acids assist to improve overall health and well-being. Emerging evidence suggests that dietary ingredients modulate gene and protein expression, thereby modify endogenous metabolic pathways and homeostasis and, consequently, reduce the risk of CVD. Given the scope and prevalence of CVDs, a population health strategy - 'prevention is better than cure' - would be the most appropriate model to adopt to deal with CVD-related mortality and morbidity.

\section{1}

PLASMA OXIDIZED PHOSPHOLIPID PROFILE AS A FUNCTION OF TIME AND FLAXSEED CONSUMPTION IN PATIENTS WITH PERIPHERAL ARTERIAL DISEASE (PAD)

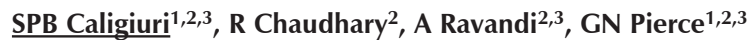
${ }^{1}$ Canadian Centre for Agri-food Research in Health \& Medicine; ${ }^{2}$ Institute of Cardiovascular Sciences, St Boniface Hospital; ${ }^{3}$ University of Manitoba, Winnipeg, Manitoba

BACKGROUND: Oxidized phospholipids (OxPL) are an emerging therapeutic target and are implicated in atherosclerosis and inflammation which are prominent in peripheral arterial disease (PAD). OxPL have yet to be investigated in PAD. Flaxseed may be a complementary treatment for $\mathrm{PAD}$, yet the influence on OxPL is unknown.

METHODS: 110 PAD patients consumed foods daily containing milled flaxseed or wheat placebo. Plasma, obtained at baseline and 6 months, underwent solid phase extraction and HPLC-MS/MS analysis.

RESULTS: Of the $80+$ plasma OxPL quantified, 20 increased over 6 months in PAD patients. Flaxseed consumption changed the type but not \# of increased plasma OxPL. The control group exhibited an increase in aldehydes, carboxylic acids and phosphatidylcholines (PCs) whereas the flaxseed group exhibited an increase in PCs and palmitoyl-linoleoylglycerophosphocholines.

CONCLUSION: Characterization of the OxPL profile provides potential therapeutic targets for PAD. Flaxseed consumption may benefit PAD patients by modifying the plasma OxPL profile. Support: CIHR, HSFC, Flax 2015, ARDI, St. Boniface Hospital Foundation

\section{2}

\section{REMOTE ISCHEMIC PRECONDITIONING AS A NOVEL STRATEGY AGAINST ISCHEMIA-REPERFUSION INJURY IN THE DIABETIC HEART}

\section{S Carnicka, I Gablovsky, M Ferko, M Murarikova, T Ravingerova} Institute for Heart Research, Slovak Academy of Sciences \& Center of Excellence SAS NOREG, Bratislava, Slovak Republic

Ischemia/reperfusion (IR) injury of the heart is often modulated by comorbities reducing effectiveness of applied therapies. Recent human and animal studies revealed protective role of remote ischemic preconditioning (RP). Present study aimed to evaluate the effects of both, early and delayed phase of RP on IR in the hearts of non-diabetic (C) and 1-week diabetic (D, streptozotocin $65 \mathrm{mg} / \mathrm{kg}$ ) male Wistar rats. RP was induced by 3 cycles of $10 \mathrm{~min}$ IR of right hind limb. Langendorff-perfused D and $C$ hearts with or without prior $\mathrm{RP}$ were exposed to $30 \mathrm{~min}$ ischemia/2 h reperfusion. Early phase of RP (1RP) was evaluated just after RP and delayed phase $24 \mathrm{~h}$ later (2RP). After IR, all studied groups exerted significant anti-infarct protection when compared to non-diabetic C. However, when comparing infarct size (IS, expressed in \% of area at risk) between $\mathrm{C}$ and $\mathrm{D}$ groups, no additional effect of $1 R P$ was found. Anti-infarct effect of $2 R P$ was attenuated in $D$ rats but was still smaller than in C. In present study, acute phase of streptozotocin-diabetes accompanied increased resistance to IR indicating adaptive process, similar to endogenous protective mechanisms induced by $1 \mathrm{RP}$ leading to an enhanced ischemic tolerance.

Funding: VEGA 2/0101/12, APVV-0102-11.

\section{3}

\section{HOW I DO MYOCARDIUM REVASCULARIZATION}

\section{E Castaneda}

Cayetano Heredia Peruvian University, Lima, Peru

In the past five decades we have witnessed a rapid development in coronary revascularization. The use of saphenous vein in coronary revascularization has been reproduced in all centers demonstrating its effectiveness. Then the use of the left internal thoracic artery has shown good results in patency and survival. However the left internal thoracic artery to the left anterior descending artery and saphenous vein graft to other vessels remains the standard operation today. The other major development has been the off-pump surgery, which provides excellent results especially in high-risk patients. The current trend is to perform complete revascularization with multiple arterial grafting and total arterial grafting. In these cases it is necessary to use bilateral internal thoracic arteries, use of the radial artery, and sequential anastomosis. The goal is to relieve symptoms and improve the prognosis of life. The management of our service is routinely used the left internal thoracic artery to the left anterior descending artery and saphenous vein (no-touch technique) to the rest of coronary arteries. In some cases, we use radial artery (young or diabetic people). Most of our patients required on-pump surgery $(62 \%)$, performing more than two coronary grafts. We performed off-pump surgery in high risk patients. In all cases, we have performed sternotomy.

\section{4}

DOXORUBICIN-INDUCED ACTIVATION OF INTRACELLULAR MATRIX METALLOPROTEINASE-2 BY OXIDATIVE STRESS IN CARDIOMYOCYTES AND HT1080 CELLS

\section{B Chan, B Hughes, R Schulz}

Departments of Pediatrics \& Pharmacology, University of Alberta, Edmonton, Alberta

Matrix metalloproteinase-2 (MMP-2) is involved in many oxidative stress associated pathologies including myocardial ischemia-reperfusion injury. MMP-2 is also an intracellular protease with specific intracellular substrates including troponin I. Doxorubicin, an anticancer drug, is cardiotoxic in part because it acutely stimulates peroxynitrite biosynthesis. We hypothesize that doxorubicin activates intracellular MMP-2 activity. To test this, neonatal rat ventricular myocytes (NRVM) and human fibrosarcoma cells (HT1080 cells) were treated with doxorubicin for $0.5 \mathrm{~h}$ and $2 \mathrm{~h}$ at $37^{\circ} \mathrm{C}$. In HT1080 cells, gelatin zymography revealed a 1.5-fold increase in intracellular MMP- 2 activity after $2 \mathrm{~h}$ without any change in protein level. Doxorubicin had no effect on extracellular MMP-2. Experiments with NRVM are in progress. To visualize changes in intracellular MMP-2 activity in real time, we will use a genetically encoded fluorescence resonance energy transfer (FRET)-based biosensor. This construct contains the MMP-2-selective cleavage site in troponin I genetically fused between two fluorescent proteins. The FRET-based biosensor will allow us to better understand how oxidative stress affects intracellular MMP-2 activity and its specific substrates.

\section{5}

\section{MANAGEMENT OF CARDIOVASCULAR DISEASE WITH OMEGA-3 FATTY ACIDS}

SK Cheema, B Kayode

Department of Biochemistry, Memorial University, St John's, Newfoundland and Labrador

Genetics, diet and life style are the predominant factors contributing towards an increased risk of cardiovascular disease (CVD). The recent phenomenon of "Developmental origins of health and diseases" places significant importance on maternal diet in predisposing the next generation to metabolic disorders. Dietary saturated fats (SFA) are generally associated with an increased risk of CVD; however diets enriched in omega (n)-3 polyunsaturated fatty acids (PUFA) protect against CVD. There is limited information on the role of maternal dietary n-3 PUFA in "in-utero programming". We have shown that a maternal diet high in SFA increased the levels of lowdensity lipoprotein (LDL) cholesterol of the offspring by inhibiting hepatic LDL-receptor gene expression. Furthermore, maternal high fat diets caused aortic endothelial dysfunction, which is an additional factor associated with CVD. On the other hand, maternal diets high in n-3 PUFA reduced plasma lipid levels of the offspring, thereby reducing the risk of CVD. The effect of maternal diets enriched in n-3 PUFA on plasma lipid levels was dose and sex specific, where longer and sustained exposure to higher levels of n-3 PUFA showed a greater reduction in plasma lipid levels.

Funding: NSERC. 


\section{6}

\section{GENETICS OF SUDDEN CARDIAC DEATH AND ROLE OF THE MOLECULAR AUTOPSY}

\section{SS Chugh, K Reinier, A Uy-Evanado, C Teodorescu,}

A Huertas-Vazquez

Cedars-Sinai Heart Institute, Los Angeles, California, USA

Sudden cardiac death (SCD) remains a global public health problem. Current methods of SCD risk prediction are inadequate and there is a significant need to identify novel markers. While SCD is a complex condition, several studies have established a significant genetic contribution in the pathogenesis of this condition. The Oregon Sudden Unexpected Death Study (Oregon SUDS, established 2002) is an ongoing prospective community-based case-control study among a population of the approximately1 million residents of Portland, Oregon, USA metropolitan area. A bio-bank (DNA, blood, tissue) of $>4000$ patients is linked to a detailed clinical phenotyping database with $>700$ variables, for the purpose of identifying novel clinical and genetic predictors. From genome-wide analyses as well as candidate gene studies we have identified several loci associated with increased risk of SCD. At the present time, use of genetic variation for risk stratification requires that a clear separation be made between common and rare forms of SCD. In specific instances, the molecular autopsy can greatly facilitate prediction and prevention of SCD.

Acknowledgements: Funded by grants from the US National Heart Lung and Blood Institute to SSC.

\section{7}

\section{TRANSCRIPTIONAL CONTROL OF CARDIAC} EXTRACELLULAR MATRIX GENE EXPRESSION

\section{Czubryt, R Bagchi, P Roche}

\section{University of Manitoba, Winnipeg, Manitoba}

The cardiac extracellular matrix (ECM) provides critical physical and functional support to the heart, however excessive matrix production fibrosis - results in impaired pump function, arrhythmogenesis and failure. The TGF $\beta$-Smad signaling pathway is considered to be the most potent regulator of ECM production, but the role of other transcription factors is less understood. Our work has revealed that scleraxis is required for normal cardiac ECM production - gene deletion of scleraxis results in loss of nearly $50 \%$ of ECM gene expression, including fibrillar collagens and various proteoglycans and matrix metalloproteinases. These animals present with echocardiographic signs of early systolic dysfunction at 6 weeks of age, and evidence that nearly half of the normal complement of cardiac fibroblasts has been lost. Our data show that scleraxis transcriptionally regulates a number of key ECM genes encoding collagens types I and III, MMP2, and numerous markers of cardiac fibroblasts and myofibroblasts using gain and loss of function coupled with rescue experiments. Overexpression of scleraxis promotes phenoconversion to myofibroblasts, in part due to transactivation of the a-smooth muscle actin gene promoter. These results reveal that scleraxis is a novel profibrotic transcription factor and potential target for fibrosis therapy.

\section{8}

\section{"MITOMIR": A NEW PLAYER IN HEART FAILURE}

\section{$\underline{\text { S Das, }}$ C Steenbergen}

\section{Johns Hopkins University, Baltimore, Maryland, USA}

Here, we explore the novel concept that miRNA can regulate heart mitochondrial biology by repressing the expression of target genes by posttranscriptional regulation. We showed that miRNA, derived from the nuclear genome, translocates to the mitochondria, and regulates mitochondrial gene expression and affects mitochondrial function. Acute overexpression of miR-181c results mitochondrial complex IV remodeling and chronic overexpression of miR-181c leads to heart failure by complex IV dysfunction. Mitochondria have their own genome, and some of their most critical proteins are encoded by mitochondrial genes. Coordination of nuclear gene expression and mitochondrial gene expression is thus essential. Our work shows that miR-181c binds to the 3'-end of the mRNA of a mitochondrial gene, mt-COX1, and initially results in complex IV remodeling, with increased production of ROS. But prolonged miR-181c overexpression alters the mRNA levels of selective mitochondrial complex IV genes, suggesting selective dysfunctional complex IV due to miR-181c targeting mt-COX1. Isolated heart mitochondrial studies showed significantly altered $\mathrm{O}_{2}$-consumption, $\mathrm{ROS}$, matrix $\mathrm{Ca}$, and in miR-181c-treated animals. Thus we propose "MitomiR" dysregulation leads to cardiac dysfunction by regulating mitochondrial genes.

Acknowledgements: Grant funding provided by the American Heart Association to SD.

\section{9}

\section{BONE MARROW CELL THERAPY IN ISCHEMIC HEART} DISEASE: EVIDENCE FROM CLINICAL TRIALS

\section{B Dawn}

University of Kansas Medical Center, Andover, Kansas, USA

Based on promising data from preclinical studies, numerous clinical trials of cardiac repair with adult bone marrow cells (BMC) have been completed over the past decade. These early trials included relatively small numbers of patients and injected widely variable numbers of cells using different routes at various time-points after myocardial infarction (MI). Understandably, the outcomes of BMC therapy in these trials have been disparate. Because of significant inter-study variability in observations, pooled data from these studies have been subjected to numerous metaanalyses over the past several years. Although these meta-analyses included variable combinations of trials and patients, the results generally indicate that BMC therapy in patients with acute MI and chronic IHD produces modest improvements in left ventricular function and structure without any increase in adverse effects. Nonetheless, a number of significant challenges remain to be overcome in order to realize the full potential of BMC therapy in clinical practice. The purpose of this talk is to summarize the available clinical evidence regarding the efficacy and safety of therapeutic cardiac repair with adult BMCs, and to discuss insights from meta-analyses on this topic.

Acknowledgments: Supported, in part, by NIH grant R01 HL-117730.

\section{0}

SARCOLEMMAL MEMBRANE ASSOCIATED PROTEIN (SLMAP) AS A NOVEL REGULATOR OF GLUT4 EXPRESSION AND TRAFFICKING

A Dewan $^{1}$, M Salih ${ }^{1}$, C Triggle ${ }^{2}$, H Ding ${ }^{2}$, B Tuana ${ }^{1}$

1University of Ottawa, Ottawa, Ontario; ${ }^{2}$ Weill Cornell Medical College, Doha, Qatar

The glucose transporter 4 (GLUT4) is critical for glucose transport into cells and its regulation and trafficking is altered in diabetes and cardiomyopathy. Akt, a protein kinase, is required for the trafficking of GLUT4 within the cell and Akt deficiency is linked to insulin resistance in type 2 diabetes. Sarcolemmal membrane associated protein isoform 1 (SLMAP1) expression has been shown to be regulated in vascular and adipose tissue in diabetic animals and its expression correlates with GLUT4 expression in diabetic mice. Here we have assessed the role of SLMAP1 on GLUT4 trafficking within the hearts of mice genetically engineered to overexpress SLMAP1. Trangenic (tg) hearts present with cardiomyopathy and both GLUT4 and Akt expression was markedly upregulated in cardiomyocytes from SLMAP1 tg mice compared with wildtype littermates. Co-localization of SLMAP1 with GLUT4 was found in vesicle compartments as well as a drastic increase in the size of these vesicles in tg cardiomyocytes. This data supports a role for SLMAP in glucose metabolism and transport in the myocardium and implies that SLMAP may be a unique target in preventing diabetic cardiomyopathy.

Acknowledgements: Grant funding from the Heart and Stroke Foundation.

\section{1}

LOSS OF MITOCHONDRIAL UCP3-CYTOCHROME C OXIDASE COMPLEXES UNDERLIES MALADAPTIVE AUTOPHAGY AND CARDIAC FAILURE IN ANTHRACYCLINE CARDIOTOXICITY R Dhingra, J Lin, D Jassal, G Dorn II, LA Kirshenbaum St Boniface Research Centre, Winnipeg, Manitoba

In this report, we provide evidence for a novel signaling pathway that functionally links activation and mitochondrial targeting of the Bcl-2 
death protein Bnip3 to doxorubicin (dox) cardiotoxicity. Perturbations to mitochondria including increased calcium, ROS, loss of DYm and mPTP opening were observed in cardiac myocytes treated with dox. This coincided with a decline in maximal respiratory capacity, loss of respiratory chain complexes of uncoupling protein 3 (UCP3) and cytochrome $c$ oxidase complex IV subunit 1, (COX) and cell viability. Mitochondrial dysfunction was accompanied by an increased autophagy and necrosis. Inhibition of autophagy with 3-methyl adenine (3-MA), or Atg7 knockdown suppressed necrotic cell death induced by dox and restored UCP3. COX complexes, Finally, mice germ-line deficient for Bnip3 were resistant to dox induced injury. Hence, this study reveals that the cardiotoxic effects of dox are mutually dependent and obligatorily linked to Bnip3-induced maladaptive autophagy and necrotic cell death.

\section{2}

CONTROLLED RELEASE OF PGE2 BY HYDROGEL PREVENTS REJECTION OF ALLOGENEIC STEM CELLS AND RESTORES POST-INFARCTION VENTRICULAR FUNCTION

S Dhingra ${ }^{1}$, P Li' ${ }^{2}$, XP Huang ${ }^{2}$, J Guo ${ }^{2}$, J Wu² ${ }^{2}$ A Mihic ${ }^{2}$, SH Li², WF Zang², RD Weisel ${ }^{2}, \mathrm{RK} \mathrm{Li}^{2}$

${ }^{1}$ St Boniface Research Centre, University of Manitoba, Winnipeg, Manitoba; ${ }^{2}$ McEwen Centre for Regenerative Medicine, University of Toronto, Toronto, Ontario

Allogeneic mesenchymal stem cells (MSCs) are immunoprivileged and are currently under investigation in clinical trials. We confirmed that allogeneic MSCs improved heart function after implantation in the infarcted heart. However, late after implantation, MSCs lost their immunoprivilege, and were rejected. The present study provides mechanisms of rejection and a way to prevent it. We demonstrated that immunoprivilege of MSCs was maintained by prostaglandin E2 (PGE2), produced by these cells. We further demonstrated that myogenic differentiation of MSCs led to PGE2 decrease and loss of immunoprivilege. PGE2 treatment of differentiated MSCs preserved immunoprivilege. Using an in vivo rat model, MSCs $\left(3 \times 10^{6}\right.$ cells/rat $)$ with or without a biodegradable hydrogel that slowly released PGE2 were injected into infarct region. After 5 weeks, significant number of transplanted cells survived in hydrogel+PGE2 group along with improvement in heart function. In conclusion, maintaining PGE2 levels in differentiated MSCs preserved immunoprivilege and restored cardiac function after MI. Supported by CIHR.

\section{3}

ONE YEAR DIETARY FLAX SUPPLEMENTATION DID NOT AFFECT CVD, INFLAMMATORY AND RENAL FUNCTION MARKERS IN PATIENTS WITH PAD AND DID NOT IMPROVE DIABETIC SUBGROUPS GLUCOSE LEVELS

E Dibrov $^{1}$, TG Maddaford ${ }^{2}$, A Edel ${ }^{2}$, A Rodríguez Portelles ${ }^{3}$, D Rodriguez Leyva $^{3}$, R Guzman ${ }^{1}$, GN Pierce ${ }^{12}$

${ }^{1}$ CCARM, St Boniface Hospital; ${ }^{2}$ University of Manitoba, Winnipeg, Manitoba; ${ }^{3}$ VI Lenin Universitary Hospital, Holguin, Cuba

Flaxseed is a great source of a-linoleic acid, dietary fiber, a third of which is soluble, and is the richest source of dietary lignans - phytoestrogens. All of these compounds have been linked to reduce cholesterol; improve insulin sensitivity; lowering glucose and reduce risk of CVD. We conducted a double blinded, randomized, placebo controlled study to assess the dietary flaxseed supplementation on cardiovascular health in patients with PAD. Significant decrease in systolic and diastolic blood pressure was detected during the trial. Multiplex analysis and assays of various biomarkers for CVD, inflammation and kidney function revealed no changes in the flax group versus the placebo group. No changes in initial and absolute claudication distances were observed. Glucose level in plasma did not improve in PAD patients with diabetes. These data suggest that renal function and inflammation may not play an important role in decreasing systolic and diastolic blood pressure detected as a result of the dietary intervention with flaxseed.

Funding: Flax2015, SBHF, Canada Bread, Canola Council of Canada, CIHR \& ARDI.

\section{4}

\section{THE DEVELOPMENTAL ORIGINS OF CARDIAC HYPERTROPHY}

\section{VW Dolinsky}

Diabetes Research Envisioned and Accomplished in Manitoba (DREAM) Theme, Manitoba Institute of Child Health, University of Manitoba, Winnipeg, Manitoba

BACKGROUND: During late prenatal and early postnatal life, growth of the heart switches from hyperplastic to hypertrophic growth suggesting that cardiac size may be permanently programmed in the early life environment. Left ventricular hypertrophy (LVH) influences the development of diabetic cardiomyopathy, though the role of gestational diabetes mellitus (GDM) is unknown.

HYPOTHESIS: Exposure to GDM increases the susceptibility of offspring to LVH. Methods: We utilized a rat model of high fat (45\%) and sucrose (HFS) diet-induced GDM. Ultrasound technology was used to analyze cardiac morphology and function in rats exposed to GDM.

RESULTS: Consumption of HFS diets by pregnant rats caused elevated gestational weight gain, hyperglycemia and hyperinsulinemia, characteristic of GDM. The LV posterior wall and heart weight:body weight ratio was 1.3 -fold $(\mathrm{P}<0.01)$ greater in fetal offspring from GDM mothers compared to lean mothers. The postnatal consumption of HFS diets by offspring from GDM mothers increased the postnatal heart weight:tibia length ratio 1.3fold $(\mathrm{P}<0.05)$ compared to offspring from lean mothers.

CONCLUSION: GDM caused LVH in rat offspring that may contribute to the early onset of diabetic cardiomyopathy.

\section{5}

\section{MICRORNA CONTROL OF PACEMAKING IN THE TRAINED HEART}

\section{A D'Souza, J Elliott, H Dobrzynski, D Oceandy, MR Boyett} University of Manchester, Manchester, United Kingdom

Athletes are prone to bradyarrythmias but the underlying mechanisms are poorly understood. In previous work we demonstrated training-induced electrical remodelling of the sinoatrial node $(\mathrm{SN})$, including a downregulation of the key pacemaker channel HCN4. Here we investigated post transcriptional regulation of $\mathrm{HCN} 4$ by microRNAs (miRNA) in the trained SN. Mice subjected to 60-minute swimming twice daily for 28 days (TM) were compared to parallel sedentary mice (SM). The protocol recapitulated a significant resting bradycardia in TM. Deep sequencing for miRNA carried out on SN samples revealed 37 differentially expressed miRNA in TM vs SM. Following bioinformatics screens for miRNA binding sites in the 3'UTR of the HCN4 gene, we identified miR-1, miR486-3p and miR-423-5p as possible candidates participating in the post-transcriptional repression of HCN4. Transient transfection of synthetic precursor miR-1 decreased HCN4 3'UTR reporter activity in H9C2 cells, directly demonstrating the regulation of HCN4 by miR-1. We describe a novel molecular mechanism whereby an altered miRNA profile in the trained SN promotes HCN4 downregulation and altered sinus automaticity. Data suggest miR-1 as a potential therapeutic target for bradyarrythmias.

Acknowledgements: Grant funding provided by The British Heart Foundation to MRB.

\section{6}

\section{THE ENDOVASCULAR TREATMENT OF TYPE B AORTIC DISSECTION - A LITERATURE REVIEW}

\section{Ш Duarte, JCDV Pontes}

\section{Cirurgia Cardiovascular, Brazil}

Aortic dissection is a cardiovascular event of high mortality if not diagnosed early and treated properly. In the type A aortic dissection, there is the involvement of the ascending aorta, whereas in type B the ascending aorta is not affected. The surgical treatment of type A aortic dissection is the gold standard. In type B aortic dissection, the hospital mortality of medical treatment is lower than that of surgical treatment, $10 \%$ against 20\%. However, half of patients who are discharged after medical treatment, develop aortic complications in subsequent years, and the mortality in three to five years may reach $25 \%$ to $50 \%$. Furthermore, the surgical treatment of aortic 
complications after medical treatment, also showed high mortality. Thus, endovascular treatment emerged as an alternative less invasive of treatment for this disease. Initially indicated for complicated cases only, showed a lower mortality rate than $10 \%$, with $>80 \%$ success rate of occlusion and thrombosis of the false lumen. The INSTEAD, which randomized patients with aortic dissection type $\mathrm{B}$ uncomplicated to optimal medical therapy plus endovascular treatment and to optimal medical therapy only, showed that after three years, patients who underwent endovascular treatment showed lower mortality and aorta-related complications.

\section{7}

\section{EXERCISE-MEDIATED PATHWAYS INFLUENCE SERCA2A REGULATION IN THE HEART}

TA Duhamel, SE Susser, T Moffatt, K O'Hara, A Stammers,

\section{Friesen}

University of Manitoba, Winnipeg, Manitoba

Exercise enhances myocardial calcium-cycling by improving SERCA2a expression and function in the diabetic heart. Protein acetylation/deacetylation is a post-translational modification that acutely regulates the activity of proteins. A proteomic study recently identified 3 acetylation sites within SERCA2a. This novel observation raises the possibility that the acetylation of SERCA2a may acutely regulate myocardial calcium-cycling. Therefore, we determined if changes in SERCA2a acetylation negatively influence SERCA2a function in the heart. We have data indicating that SERCA2a acetylation is increased by $56 \%$ while SERCA2a activity is reduced by $27 \%$ in hearts isolated from SIRT3 (i.e. sirtuin 3, a class III histone deacetylase) knockout mice, as compared to wild-type mice. We also have data indicating that SERCA2a acetylation is increased 2-fold following high fat feeding and normalized in exercise-trained WT mice. Finally, the acetylation of SERCA2a, which was achieved using site directed mutagenesis, directly impairs maximal SERCA2a activity by $38 \%$. We speculate that SERCA2a acetylation may directly impair myocardial calcium-cycling in the diseased heart and may also be an underlying mechanism by which exercise training enhances myocardial calcium-cycling.

Acknowledgements: Grant funding provided by HSFC and CIHR.

\section{8}

DIETARY FLAXSEED IMPROVES LIPID LOWERING IN PATIENTS ALREADY USING STATINS

A Edel ${ }^{1}$, D Rodriguez-Leyva ${ }^{2}$, T Maddaford ${ }^{1}$, S Caligiuri ${ }^{1}$, R La Vallée ${ }^{1}$, W Weighell ${ }^{3}$, R Guzman ${ }^{3}$, M Aliani ${ }^{4}$, G Pierce ${ }^{1}$ ${ }^{1}$ Cell Biology Laboratory, CCARM, SBGH, Department of Physiology, University of Manitoba, Winnipeg, Manitoba; ${ }^{2}$ Cardiovascular Research Division, VI Lenin Universitary Hospital, Holguin, Cuba; ${ }^{3} \mathrm{ACRI}, \mathrm{SBGH}$, and the Department of Surgery; ${ }^{4}$ Department of Human Nutritional Sciences, University of Manitoba, Winnipeg, Manitoba

Nearly $67 \%$ of US adults with high cholesterol do not have normal levels despite current treatments. Dietary flaxseed can lower cholesterol in healthy adults, however its effects with administered cholesterol-lowering medications (CLMs) have not been investigated. Our objective was to see if flaxseed could boost the effects of CLMs in unhealthy patients with CVD. The Flax-PAD trial involved 110 patients with peripheral arterial disease. Patients were randomized into a flaxseed (FX) or whole-wheat (PL) group. FX or PL was ingested in food products $(30 \mathrm{~g} / \mathrm{d}$ ) over 1 year. At baseline $74 \%$ of patients were on CLMs. LDL-C was attenuated in the FX+CLM group compared to PL+CLM by $-5.7 \%$ vs $0.3 \%, P=0.315$ after 6 months and after 12 months by $-8.5 \%$ vs $3.0 \%, P=0.030$. Patients adding $30 \mathrm{~g}$ of $\mathrm{FX}$ to their diet offers potential advantages in lowering LDL-C in addition to those induced by CLMs.

Acknowledgements: CIHR, Flax2015, Heart and Stroke Fdn of Canada, SBGH Foundation and the Canola Council.

\section{9}

\section{BENEFICIAL EFFECTS OF $\mathrm{CO}_{2}$-ENRICHED WATER BATH TREATMENT ON BLOOD FLOW AND ANGIOGENESIS IN ISCHEMIC HIND LIMB}

V Elimban, YJ Xu, NS Dhalla

Institute of Cardiovascular Sciences, St Boniface Hospital Research

Centre, Winnipeg, Manitoba

Peripheral artery disease (PAD) is a major health problem whereby narrowed arteries reduce blood flow to the limbs. We investigated the effects of $\mathrm{CO}_{2}$-enriched water bath $(\mathrm{CEWB})$ treatment on blood flow in the ischemic hind limb. The femoral artery was occluded in rats to induce PAD. Rats were treated with or without CEWB at $37^{\circ} \mathrm{C}$ for 4 weeks (20 min/day; 5 days/week) starting one week after artery occlusion. CEWB was prepared by using Carbothera (Mitsubishi Rayon Engineering, Tokyo). Peak, mean or minimal blood flow, measured by Pulse Wave Doppler Ultrasound technique, was not detected in the untreated ischemic hind limb animals due to arterial ligation. However, blood flow values were about $50 \%$ of the control upon treatment with CEWB; $67 \%$ of the ligated animals showed positive blood flow by $\mathrm{CO}_{2}$ treatment. Morphological examination of the treated ischemic skeletal muscle revealed a 3-fold increase in small artery numbers. Although plasma triglycerides decreased and plasma NO concentration increased in ischemic animals, CEWB treatment produced no effects on these parameters. It is suggested that the beneficial action of $\mathrm{CO}_{2}$ therapy on blood flow to hind limb may be due to the formation of new blood vessels in the ischemic skeletal muscle.

\section{0}

\section{ROLE OF CAMKII BETA ISOFORM IN DILATED CARDIOMYOPATHY AND HEART FAILURE}

Y Fanous, MT Sonoda*, F Gaisler da Silva*, J Major, M Salih, M Ramos, BS Tuana

Department of Cellular Molecular Medicine, University of Ottawa, Ottawa, Ontario

The calcium calmodulin dependent protein kinase II $\beta 4$ (CaMKII $\beta 4$ ) is a muscle-specific isoform of the CaMKII family of kinases expressed in cardiomyocytes. Although the involvement of CAMKII $\delta$ isoform in calcium transport and cardiac remodeling is well known, the role of CaMKIIP4 in myocardium remains to be defined. Transgenic ( $\mathrm{tg}$ ) mice with cardiac specific overexpression of CaMKII $\beta 4$ were generated and examined with echocardiography to assess functional parameters, molecular parameters were analyzed using qPCR and Western blotting. CaMKII $\beta 4$ overexpression in heart of transgenic mice resulted in marked changes in both structural and functional parameters along with changes in the phosphorylation status of major $\mathrm{Ca}^{2+}$ handling proteins. Overexpression of CaMKII $\beta 4$ resulted in severe and rapidly developing heart failure, indicating the enzyme's potency in cardiac biology. The results indicate that aberrant CaMKII $\beta 4$ activity in myocardium results in a dilated cardiomyopathy phenotype which is characteristically different from that observed in CAMKIII isoform overexpression hearts. Thus isoform specific CAMKII targeting may represent unique therapeutic opportunities in cardiac pathology.

Acknowledgements: Funded by CIHR. *Supported by CAPES

\section{1}

VASCULAR EFFECTS OF STEROIDS: SORTING OUT THE RECEPTORS AND THE LIGANDS

\section{RD Feldman, R Gros}

Western University, London, Ontario

The traditional view of the cardiovascular actions of estrogen and aldosterone has focused on their roles as regulators of transcription via activation of their 'classical' receptors MR and ERs However, scientists have long speculated that estrogen and aldosterone might have effects on regulation of smooth muscle contractility, cell growth and differentiation that are too rapid to be accounted for by transcriptional regulation. Recent studies have begun to elucidate the mechanism of rapid steroid-mediated cardiometabolic regulation. GPR30, now designated as GPER, a newly characterized 'orphan receptor', has been implicated in mediating the rapid effects of estradiol and most recently those of aldosterone. Studies 
to date have taught us that to understand the rapid vascular mechanisms of steroids, one must (i) know which vascular 'compartment' the steroid is acting and (ii) know which receptor the steroid hormone is activating; and (iii) not assume the receptor specificity of a steroid receptor ligand based solely on its selectivity selectivity for its traditional 'transcriptional' steroid receptor.

\section{2}

\section{ABERRANT REGULATION OF THE NA+/H+ EXCHANGER EXACERBATES DAMAGE TO THE MYOCARDIUM IN HEART DISEASE}

\section{Fliegel}

Depart of Biochemistry, University of Alberta, Edmonton, Alberta

$\mathrm{The} \mathrm{Na}+\mathrm{H}+$ exchanger isoform 1 (NHE1) is a plasma membrane $\mathrm{pH}$ regulatory protein that removes one intracellular $\mathrm{H}+$ in exchange for an extracellular $\mathrm{Na}+$. It has a 500 amino acid $\mathrm{N}$-terminal membrane domain and a 315 amino acid, C-terminal cytosolic domain. In heart disease, ischemia/reperfusion activates NHE1 and this leads to excess sodium uptake which leads to excess intracellular calcium via the sodium/calcium exchanger. We determined that Ser770 and Ser771 were necessary for NHE1 activation. To determine how phosphorylation activates the protein we studied the conformational changes on the regulatory tail. Tryptic digestion of the C-terminal regulatory region showed that a phosphomimetic protein had altered conformation. Tryptophan fluorescence indicated that wild type and phosphomimetic purified $\mathrm{C}$-terminal region had $\mathrm{pH}$-dependent differences in the conformation. Bottom-up hydrogen/deuterium exchange mass spectrometry demonstrated that a peptide fragment containing phosphomimetic mutations became strongly stabilized relative to the wild type protein. The results show that phosphorylation of S770/S771 changes the conformation of the $\mathrm{C}$-terminal regulatory region in a $\mathrm{pH}$-dependent manner, resulting in a more compact region that increases NHE1 activity in the diseased myocardium. Funding: Supported by CIHR.

\section{3}

\section{EX VIVO PERFUSION AS A METHOD FOR DONOR HEART TREATMENT AND ASSESSMENT}

\section{DH Freed}

Departments of Surgery and Physiology, University of Alberta,

\section{Edmonton, Alberta}

Cardiac transplantation remains the gold standard therapy for the definitive treatment of end-stage heart failure. However, application of this therapy is limited by a small number of good quality donor organs. Ex vivo organ perfusion has been proposed as a method to expand the pool of donor organs for transplantation. Devices have been developed and are currently either in routine clinical use, as in the case of kidney, or are under clinical investigation as in the case of lung and liver. We have developed a device that allows full biventricular working mode analysis of donor cardiac function. This device is fully self-contained, eliminating the need for separate dedicated pressure-volume (PV) loop analysis equipment. The utility of the device to accurately and reproducibly assess function was confirmed through comparative analyses with standard PV loop equipment. We then demonstrated the need for functional analysis, as other parameters such as lactate, do not correlate well with myocardial performance. Optimal conditions for normothermic ex vivo preservation of the donor heart, including hemodynamic parameters and perfusate composition are being determined. In summary, ex vivo heart perfusion show great promise as a method for increasing the number and quality of donor hearts for transplantation.

\section{4}

\section{NOVEL TARGETS AND THEIR POTENTIAL FOR METABOLIC SYNDROME}

\section{TR Gandhi}

\section{Anand Pharmacy College, Anand, India}

Metabolic syndrome is a cluster of risk factors like central obesity, hypertension, impaired glucose tolerance, insulin resistance and dyslipidemia. It is on the rise globally, and due to incompletely revealed underlying pathophysiology of complications, no standard therapy is yet available. Alterations in fat metabolism, oxidative stress and inflammatory changes could be a few of the major contributing factors of metabolic syndrome. Many molecular targets are studied to explore the underlying mechanisms in this regard, like NFB, Sterol response element binding protein 1-c, Nuclear factor E2 related factor -2 , Kelch-like ECH associated protein 1, PPAR- $\gamma$, fatty acid binding protein 4, cluster of differentiation 36, LPL, Steroyl-CoA desaturase, Acetyl-CoA carboxylase 1, etc. Metabolism of substrates of CYP450 isoenzymes is also reported to be modulated during various metabolic disorders. Expression of CYP2E1, CYP1A2, CYP2C9, CYP2C19, CYP3A4 and CYP2D6 are reported to be altered in obese condition. Further, disruption of CYP4A14 gene has shown to cause hypertension resembling human hypertension and inhibitors of CYP4A family may have therapeutic benefit in management of diabetic nephropathy. CYP450 is expected to be a novel and potential target; modulation in its expression can be beneficial in prevention of metabolic syndrome and its complications.

\section{5}

\section{ROLE OF THE PARASYMPATHETIC NERVOUS SYSTEM IN CARDIOPROTECTION BY REMOTE HINDLIMB ISCHAEMIC PRECONDITIONING}

R] Gelpi, M Donato, B Buchholz, M Rodriguez

Cardiovascular Physiopathology Institute, Faculty of Medicine, Buenos Aires University, Buenos Aires, Argentina

The goal was to determine the participation of the vagus nerve and muscarinic receptors in the remote ischaemic preconditioning (rIPC). New Zealand rabbits were anaesthetized, and the femoral artery was dissected. The hearts were isolated and subjected to $30 \mathrm{~min}$ of ischaemia and $180 \mathrm{~min}$ of reperfusion (non rIPC group). In the rIPC group, three cycles of hindlimb ischaemia $(5 \mathrm{~min})$ and reperfusion $(5 \mathrm{~min}$ ) were performed, and the same protocol as that used in non-rIPC group was then repeated. The femoral and sciatic nerves were sectioned and in other group the spinal cord was sectioned (afferent pathway). The vagus nerve was also sectioned and, in another group, atropine was administered (efferent pathway). The effect of vagal stimulation was also evaluated. Infarct size was $40.8 \pm 3.1 \%$ in the nonrIPC group and $16.4 \pm 3.5 \%$ in $\operatorname{rIPC}(\mathrm{P}<0.05)$. During the $\mathrm{rIPC}$ protocol, the vagus nerve section and atropine abolished the effect of rIPC on infarct size. Vagal stimulation also decreased infarct size to $15.2 \pm 4.7 \%(\mathrm{P}<0.05)$, and the spinal cord section completely abolished the effect of rIPC. Thus, the cardioprotective signal reaches the heart through the vagus nerve, and muscarinic receptors activate ischaemic preconditioning.

\section{6}

EXTENSIVE AUTOLYTIC FRAGMENTATION OF MEMBRANOUS VERSUS CYTOSOLIC CALPAIN FOLLOWING MYOCARDIAL ISCHEMIA-REPERFUSION (I/R)

ISC Gilchrist, T Cook, B Abrenica, B Rashidkhani, GN Pierce Institute of Cardiovascular Sciences, St Boniface Hospital Research Centre, University of Manitoba, Winnipeg, Manitoba

Calpain activation was investigated following $\mathrm{I} / \mathrm{R}$ of rat hearts by Western blot detection of its own autolytic and calpain substrate fragmentation in cytosolic and membrane fractions. After I/R, the catalytic calpain subunit was autolytically fragmented to 56 and $43 \mathrm{kDa}$ peptides primarily in membrane fractions. This accompanied calpain-like degradation of membraneassociated $240 \mathrm{kDa} \alpha$-fodrin to $150 / 145 \mathrm{kDa}$ signature peptides. In contrast, cytosolic calpain remained largely intact with only weak immunostaining of autolytic peptide fragments. Myofibrillar $\alpha$-fodrin was degraded but without a characteristic calpain-induced degradation pattern. In purified SR membranes, RyR2 and SERCA2 proteins were also highly degraded again without production of calpain-induced signature peptides. When I/R-treated hearts were perfused with peptidyl calpain inhibitors, calpain autolysis and $\alpha$-fodrin degradation were robustly attenuated. However, inhibitors only weakly protected against early loss of developed pressure following $\mathrm{I} / \mathrm{R}$. Our data suggest calpain is preferentially activated at membranes following $\mathrm{I} / \mathrm{R}$ but it may only contribute a small amount to IR injury.

Funding: CIHR 


\section{7}

NEW ELECTROPHYSIOLOGICAL AND TECHNICAL APPROACH FOR ATRIAL FIBRILATION TREATMENT DURING MITRAL VALVE SURGERY

\section{OM Gomes}

Sao Francisco de Assis Truth is Jesus Cardiovascular Foundation, Minas Gerais, Brazil

OBJECTIVE: To describe a new electrophysiological and technical approach for atrial fibrilation treatment during mitral valve surgery.

METHODS: Twenty patients with chronic atrial fibrillation and mitral valve dysfunction and with tricuspid valve regurgitation in one case were operated on. The following modifications of the classic Cox procedure were employed: 1 . Exclusion of the left atrium appendage by internal suturing to close its left atrial ostium; 2. continuous suture of one $\mathrm{cm}$. extension at the anterior wall contour of superior and inferior vena cava atrium junction extremities; 3 . Exclusion of the right atrium appendage by one purse string suture used for the fixation of the superior vena cava draining cannula; 4. Single atrial incision; 5. Transendocardial electrocauterization of the left atrium wall around all the pulmonary vein ostia; 6 . Substitution of the incisions and sutures in the left atrium by transendocardial electrocauterization; and, 7. Nakagawa re-entry circuit cauterization, in one case. RESULTS: All patients were in regular atrial rhythm at the end of surgery and the postoperative period was uneventful.

\section{8 \\ SWERTIAMARIN: POSSIBLE ANSWER FOR DIABETIC CARDIOMYOPATHY}

\section{RK Goyal, N Patel, V Khanna}

\section{Institute of Life Sciences, Ahmedabad University, India}

In diabetic cardiomyopathy, there occurs imbalance between uptake and utilization of lipids leading to an accumulation of lipids in cytoplasm in the form of long-chain acyl-carnitines. Increase in fatty acid oxidation causes an alteration in acetyl co-A-carboxylase that inhibits glucose oxidation. Fatty acids bind and activate PPARs of which PPAR- $\alpha$ is the key isoform in the heart. Swertiamarin, an iridoglycoside, from a plant Enicostemma littorale has emerged as the lead compound for anti-diabetic and anti-obesity activities. Preliminary studies have also shown that swertiamarin may prevent diabetesinduced cardiomyopathy. Further studies using 3T3L1 cell lines suggest acetyl-coA-carboxylase as the possible target for the prevention of diabetes induced cardiac complications. In silico docking studies also revealed binding of swertiamarin to the enzyme acetyl co-A-carboxylase. The interactions involved in this binding included formation of hydrogen bond between hydroxyl oxygen of Serine (1856 position) and the H43 of swertiamarin, as well as between the hydroxyl hydrogen of Threonine (1985 position) and O9 of swertiamarin. Considering the holistic effects of Enicostemma littorale, and swertiamatin, it can be explored for the development of effective treatment for diabetic cardiomyopathy.

\section{9}

THE CARDIOPROTECTIVE ROLE OF NACA IN DOXORUBICIN AND TRASTUZUMAB MEDIATED CARDIAC DYSFUNCTION

V Goyal ${ }^{1}$, KA Bordun ${ }^{1}$, S Premecz ${ }^{1}$, D Cheung ${ }^{1}$, R Fandrich $^{1}$,

B Nickel ${ }^{1}$, S Mandal ${ }^{1}$, E Kardami ${ }^{1}$, PK Singal ${ }^{1}$, S Niraula ${ }^{2}$, DS Jassal ${ }^{1,3}$

${ }^{1}$ ICS, St Boniface Research Centre; ${ }^{2}$ Medical Oncology, Cancer Care Manitoba; ${ }^{3}$ Section of Cardiology, Internal Medicine, University of Manitoba, Winnipeg, Manitoba

OBJECTIVE: To investigate whether an anti-oxidant, $\mathrm{N}$-acetylcysteine amide (NACA), can attenuate the drug-induced heart failure caused by DOX+TRZ.

METHODS: 100 female mice received one of the drug regimens: i) $0.9 \%$ saline; ii) NACA; iii) DOX; iv) TRZ; v) DOX+TRZ; vi) NACA+DOX; vii) NACA+TRZ; and viii) NACA+DOX+TRZ. LV systolic function was assessed using echocardiography. At day 10 , mice were euthanized and hearts were measured for oxidative stress (OS).

RESULTS: In mice receiving DOX, left ventricular ejection fraction (LVEF) decreased from $73 \pm 4 \%$ to $43 \pm 2 \%$ at day 10 . In mice receiving DOX+TRZ, LVEF decreased from $72 \pm 3 \%$ to $32 \pm 2 \%$ at day 10 . Prophylactic administration of NACA to mice receiving DOX or DOX+TRZ was cardioprotective with an LVEF of $62 \pm 3 \%$ and $55 \pm 3 \%$ at day 10 , respectively. There was a 3 -fold and 4 -fold increase in superoxide production in hearts of mice treated with DOX or DOX+TRZ, respectively. Prophylactic administration of NACA reduced degree of OS in both groups.

CONCLUSION: NACA attenuates the cardio-toxic effects of DOX+TRZ in a murine model of chemotherapy induced cardiac dysfunction.

\section{0}

\section{CODE STEMI: THE EVOLUTION OF PREHOSPITAL STEMI MANAGEMENT}

\section{R Grierson}

\section{St Boniface Hospital, Winnipeg, Manitoba}

This presentation will provide a review, update and data on two important joint initiatives between the Winnipeg Fire Paramedic Service (WFPS) and the Winnipeg Regional Health Authority (WRHA) Cardiac Sciences program. The Cardiac Outcomes through Digital Evaluation (CODE) STEMI program began in July 2008 and to date has reduced reperfusion times and improved outcomes for over 1100 pre-hospital STEMI patients. The CODE STEMI Red program which began in July 2012 has similarly reduced times to reperfusion for STEMI patients who initially presented to hospitals without PCI capability.

\section{1}

\section{MECHANISMS OF MESENCHYMAL STEM CELL MEDIATED MODULATION OF POST-MYOCARDIAL INFARCTION INFLAMMATORY RESPONSE}

$\underline{\text { Q Guan }}{ }^{1,2}$, B Yang1 , Z Peng1, S Dhingra ${ }^{2}$, D Wall1,2

${ }^{1}$ Department of Immunology; ${ }^{2}$ Regenerative Medicine Program, University of Manitoba, Winnipeg, Manitoba

Post myocardial infarction (MI) inflammatory response plays an important role in the healing process. Targeting inflammatory responses has been a potential strategy for MI treatment. Mesenchymal stem cells (MSC) have the ability to suppress $\mathrm{T}$ cell function and modulate inflammatory responses. The clinical trials suggested that MSC have the potential to improve MI. We postulated that inhibitory receptors/ligands expressed on the MSC may correlate with function of MSC and suppress post-MI inflammation. Programmed cell death ligand 1 (PD-L1) expressed on MSC surface, has been shown to be involved in the suppression of T cell. Human bone marrow-derived MSC were generated in the lab. It was observed that MSC suppressed lymphocyte proliferation, differentiation of Th1 and CD8T cells in a dose dependent manner. Interestingly, high expression level of PD-L1 on MSC correlated with stronger suppression in the CFSE dilution assay. Blocking PD-L1 reversed the suppressive functions of MSC. These results indicated that the expression levels of PD-L1 on MSC correlates with suppressive functions of MSC - if so, it may be a useful surrogate potency assay that could be applied to clinical grade MSC used in management of myocardial repair.

\section{2}

\section{REGULATION OF CARDIAC FIBROBLAST ACTIVATION BY MICRORNA MEDIATED PARACRINE SIGNALING}

\section{Gupta}

Department of Physiology \& Biophysics, University of Illinois, Chicago, Illinois, USA

Understanding the regulation of cardiac fibrosis is critical for controlling adverse cardiac remodeling during heart failure. We identified miR-378 as a cardiomyocyte-abundant miRNA down-regulated in several experimental models of cardiac hypertrophy and in heart failure patients. To understand the consequence of miR-378 depletion during cardiac remodeling, we employed a LNA-modified-antimiR to target miR-378 in vivo. Results showed development of cardiomyocyte hypertrophy and fibrosis in mouse hearts which was associated with increased TGF $\beta 1$. In the conditionedmedia of miR-378-depleted cardiomyocytes, TGF 11 level was increased. Treatment of cardiac fibroblasts with miR-378-depleted-myocyte-conditioned-media activated pSMAD2/3, and induced fibrotic gene expression, an effect counteracted by TGF $\beta 1$-neutralizing antibody. Dominant negative $\mathrm{N}$-Ras or c-Jun prevented antimiR-mediated induction of TGF $\beta 1 \mathrm{mRNA}$, documenting importance of Ras and AP-1 signaling in this response. Our 
study shows that reduction in miR-378 levels during pathological conditions participates in cardiac remodeling through paracrine release of TGF $\beta 1$ from cardiomyocytes. These data implicate the presence of miR-378 in cardiomyocytes plays a critical role in the protection of neighboring fibroblasts from activation by pro-fibrotic stimuli.

\section{3}

THE MITOCHONDRIAL DEACETYLASE SIRT3 BLOCKS CARDIAC FIBROSIS INDEPENDENTLY OF REGULATING ROS SYNTHESIS

\section{MP Gupta}

Center for Cardiothoracic Research, Department of Surgery, Basic Sciences Division, University of Chicago, Chicago, Illinois, USA Cardiac fibrosis after myocardial injury is a major challenge in the medicine today to preserve heart function. At the cellular level, activation of TGF $\beta 1$ signaling is considered a major contributor of cardiac fibrosis. However, the underlying mechanism of TGF $\beta$ activation is not yet completely understood. SIRT3 is a mitochondrial deacetylase which is activated by exercise and calorie restriction. SIRT3 regulates variety of mitochondrial functions, including metabolism, ROS synthesis and apoptosis. SIRT3KO mice spontaneously develop cardiac fibrosis with age. This study was undertaken to test the hypothesis whether SIRT3 deficiency leads to transformation of cardiac fibroblasts $(\mathrm{CF})$ into myofibroblasts (myoFB) and excessive synthesis of extracellular matrix (ECM). CF obtained from adult rat hearts were subjected to SIRT3 knockdown (KD) by using siRNA. Differentiation of CF to myoFB was determined by increased expression of myoFB markers, like smooth muscle $\alpha$-actin, fibronectin and collagen 1 . The results showed robust expression of myoFB markers in SIRT3KD cells. To test whether SIRT3 deficiency contributed to this phenotype, we re-expressed SIRT3 and found that this intervention changed back myoFB phenotype of SIRT3-deficient cells to normal.

\section{4}

\section{OXIDIZED PHOSPHOLIPIDS ARE REDUCED IN ALPHA LINOLENIC ACID TREATED ADULT CARDIOMYOCYTES DURING ISCHEMIA REPERFUSION}

D Hasanally ${ }^{1,2}$, R Ganguly ${ }^{1,2,3}$, TG Maddaford ${ }^{1,2,3}$, R Chaudhary ${ }^{1}$, LA Kirshenbaum $^{1,2}$, GN Pierce ${ }^{1,2,3}$, A Ravandi ${ }^{1,2}$

${ }^{1}$ Institute for Cardiovascular Science; ${ }^{2}$ Department of Physiology, University of Manitoba; ${ }^{3}$ Canadian Centre for Agri-food Research in Health and Medicine (CCARM), Winnipeg, Manitoba

The omega-3 fatty acid, alpha linolenic acid (ALA), found in plant-derived foods has been suggested to be cardioprotective during an ischemic event. In the present study, isolated adult rat cardiomyocytes were exposed to medium containing ALA for $24 \mathrm{~h}$ and then exposed to non-ischemic (control), simulated ischemia (ISCH) or simulated ischemia/reperfusion (IR) conditions to determine the mechanism of ALA cardioprotection. Phospholipids containing ALA were significantly increased after pre-treatment. Pro-apoptotic oxidized phosphatidylcholines (OxPC), POVPC and PGPC, were significantly increased in ISCH and IR, and were reduced in ALA pre-treated cardiomyocytes. Cell death, DNA fragmentation, caspase-3 activity were also reduced in ALA treated cells. Calcium transience was analyzed but no change was demonstrated. The findings suggest that ALA protects the cardiomyocyte from apoptotic cell death during simulated ISCH and IR by inhibiting the production of specific pro-apoptotic $\mathrm{OxPC}$ species.

Acknowledgments: Grant funding from MHRC, CIHR, MMSF, HSFC and SBHF.

\section{5}

CLINICAL EFFICACY OF ARTIFICIAL CO2-ENRICHED WATER

H Hayashi $^{1}$, N Makino ${ }^{2}$, H Matsuo $^{3}$, H Kumamoto $^{4}$, Y Kumada ${ }^{5}$ ${ }^{1}$ Seijoh University, Tokai; ${ }^{2}$ Kyushu University, Beppu; ${ }^{3}$ Matsuo Medical Clinic, Osaka; ${ }^{4}$ Mitsubishi Rayon Cleansui, Tokyo; ${ }^{5}$ Matsunami General Hospital, Gifu, Japan

The $\mathrm{CO}_{2}$-enriched water has been used as a clinical application in Japan, since a device was developed to produce artificial $\mathrm{CO}_{2}$-enriched water more easily in 1997. We focused on the microcirculatory effects of artificial $\mathrm{CO}_{2}$ enriched water, and studied clinical efficacy in patients with critical limb ischemia (CLI). Initially, we showed that immersion of feet in artificial $\mathrm{CO}_{2}$-enriched water $\left(\mathrm{CO}_{2}\right.$ immersion) increased the blood flow of feet much more than with plain water even in patient with CLI, and it improved the limb preservation rate in patients without indication of revascularization. Furthermore, we clarified that $\mathrm{CO}_{2}$ immersion accelerated wound healing after lower extremities bypass surgery in CLI patients with ulcer/gangrene as an adjuvant therapy. We summarize the clinical studies, and clarify the therapeutic usefulness of $\mathrm{CO}_{2}$ immersion in CLI patients.

\section{6}

INVOLVEMENT OF HEAT SHOCK PROTEIN-60 IN VASCULAR SMOOTH MUSCLE CELL PROLIFERATION THROUGH A MODULATION OF NUCLEAR PROTEIN IMPORT

\section{TE Hedley, JF Deniset, M Hlaváčková, GN Pierce}

Institute of Cardiovascular Sciences, St Boniface Hospital Research Centre, University of Manitoba, Winnipeg, Manitoba

Heat shock protein (Hsp) 60 is a proposed mediator of vascular smooth muscle cell (VSMC) proliferation. Nuclear protein import (NPI) regulates cell growth and division by controlling translocation of transcription factors into the nucleus. This study examined whether Hsp60 can mediate cell proliferation through an NPI-dependant mechanism. Hsp60 with or without a mitochondrial targeting sequence (AdHsp60mito-) was overexpressed in primary rabbit VSMCs. Alternatively, VSMCs were treated with oxLDL following Hsp60 siRNA treatment. Both AdHsp60 and AdHsp60mitoincreased Hsp60 and PCNA expression, VSMC proliferation, and rate of NPI with respect to control. Both AdHsp60 and AdHsp60mito- induced an up-regulation of the cytosolic nuclear transport proteins Importin- $\alpha$, Importin- $\beta$ and Ran compared to control. OxLDL treatment induced increases in Hsp60 and Ran expression compared to control. However, after oxLDL treatment, Hsp60 siRNA treatment decreased the expression of Hsp60 and eliminated any of the increases in Ran expression. Therefore, nuclear protein import has an important role in VSMC proliferation, which can be modulated via an Hsp60-dependent cytosolic mechanism.

\section{7}

\section{SEX DIFFERENCES IN CALCIUM HOMEOSTASIS AND CONTRACTION IN THE HEART}

\section{RJ Parks, SE Howlett}

Departments of Pharmacology \& Medicine, Dalhousie University, Halifax, Nova Scotia

Male-female differences in mechanisms regulating $\mathrm{Ca}^{2+}$ release and contraction were investigated in murine ventricular myocytes. $\mathrm{Ca}^{2+}$ transients were smaller in myocytes from females compared to males, though $\mathrm{Ca}^{2+}$ currents were similar. This was linked to lower sarcoplasmic reticulum (SR) $\mathrm{Ca}^{2+}$ release gain and smaller $\mathrm{Ca}^{2+}$ sparks. Basal cAMP levels were lower in females and PKA inhibition (H-89) abolished sex differences in $\mathrm{Ca}^{2+}$ release. Phosphodiesterase type 4B (PDE4B) expression was higher in female ventricles, which could explain reduced cAMP levels. Further, inhibition of PDE4 (rolipram) removed male-female differences in $\mathrm{Ca}^{2+}$ release. A role for estrogen was investigated in sham-operated and ovariectomized (OVX) mice. $\mathrm{Ca}^{2+}$ transients, contractions and SR $\mathrm{Ca}^{2+}$ stores were increased by OVX and this increase was abolished by PKA inhibition. These findings suggest that estrogen suppresses SR $\mathrm{Ca}^{2+}$ release by PKAdependent mechanisms. Loss of these protective effects of estrogen may help explain the increased incidence of cardiovascular disease in postmenopausal women.

Acknowledgements: CIHR (MOP-97973).

\section{8}

THE ROLE OF ZEB2 IN PROMOTING CARDIAC FIBROBLAST TO MYOFIBROBLAST PHENOCONVERSION

F Jahan $^{1,2}$, G DeGagne ${ }^{2}$, S Rattan ${ }^{2}$, IMC Dixon ${ }^{2,3}$, JT Wigle ${ }^{1,2}$ ${ }^{1}$ Department of Biochemistry and Medical Genetics, University of Manitoba; ${ }^{2}$ Institute of Cardiovascular Sciences, St Boniface Hospital Research Centre; ${ }^{3}$ Department of Physiology and Pathophysiology, University of Manitoba, Winnipeg, Manitoba Fibrosis is the heart's key response to different stresses such as myocardial infarction, hypertension and arrhythmias. Cardiac myofibroblasts 
contribute to: extracellular matrix remodelling, scar formation, myocardial stiffening, cardiac dysfunction; and eventually to heart failure. Here we show first time that Zeb2, an epithelial-mesenchymal transition inducer, may play a critical role in the generation of myofibroblasts. Using primary rat cardiac fibroblasts, we have recently shown that ectopic expression of Zeb2 causes up-regulation of a set of myofibroblast markers. By Western blotting and confocal microscopy, we have also shown that Zeb2 is highly expressed in myofibroblast nuclei whereas its expression is lower in fibroblasts. We further aim to elucidate its role in myofibroblast proliferation, apoptosis and function in vitro. Thus findings from our study indicate a crucial role of Zeb2 in myofibroblast generation and may provide a basis for developing novel anti-fibrotic drug in the future.

Acknowledgement: Canadian Institute of Health Research (CIHR) and The Heart and Stroke Foundation.

\section{9}

\section{L-ARGININE/NO SYSTEM AND OXIDATIVE STRESS: DIFFERENT ASPECTS IN BASIC AND CLINICAL RESEARCH \\ V Jakovljevic ${ }^{1}$, V Zivkovic ${ }^{1}$, N Barudzic ${ }^{1}$, D Djuric ${ }^{2}$}

${ }^{1}$ Department of Physiology, Faculty of Medical Sciences, University

of Kragujevac, Kragujevac; ${ }^{2}$ Institute of Physiology "Richard

Burian", School of Medicine, University of Belgrade, Belgrade, Serbia

The NO system plays important role in cardiovascular homeostasis. Two crucial problems with NO are short half-life and inactivation by ROS forming RNS. Our basic research tried to assess effects of vitamins and NMDA receptor modulators on rat coronary circulation. Results showed that all applied drugs have different effects on rat heart. Clinical part treated examined parameters of oxidative stress in exercise, rheumatoid arthritis and in portal hypertension. The results in top athletes suggested that the type of sport and training affects oxidative stress. In rheumatoid arthritis, disease activity was connected with some biochemical parameters. Our findings support an association between ROS/RNS and RA and suggests that those markers may be useful in evaluating the progression of RA. Finally, patients with clinically verified portal hypertension and increased vascular resistance participated in the study. Liver diameters, portal diameters and portal flow velocities were recorded. This study approved possible role of NO system in portal hypertension and could be a useful for possible implementation of some antioxidants in the treatment of portal hypertension.

\section{0}

\section{O-GLCNAC AS A REGULATOR OF CARDIOVASCULAR PATHOPHYSIOLOGY}

\section{$\underline{\text { SP Jones }}$}

University of Louisville, Louisville, Kentucky, USA

Glucose can be metabolized through several accessory pathways. One such route, the hexosamine biosynthetic pathway (HBP), has emerged as an important, albeit indirect, regulator of cardiovascular pathophysiology. UDP, $\mathrm{N}$-acetylglucosamine (UDP-GlcNAc) is the final product of the HBP and also serves as the monosaccharide donor for the post-translational modification of proteins known as O-GlcNAc. Several laboratories have identified protein $\mathrm{O}-\mathrm{GlcNA}$ cylation as a critical response to acute stress in cardiovascular tissue. Here, we will discuss the implications for O-GlcNAcylation in cardiovascular disease, including ischemia-reperfusion injury, hypertrophy, and hypertrophy. In addition, we will address novel mechanisms of regulation (and feedback) for protein O-GlcNAcylation in the heart.

\section{1}

\section{AGING AND HEART FAILURE: UPDATE 2014}

\section{B Jugdutt}

University of Alberta, Edmonton, Alberta

The aging population with heart failure (HF) is increasing globally. Morbidity and mortality from hypertension (HTN) and myocardial infarction (MI) are leading causes of HF in the elderly (age $\geq 65$ years). Cardiovascular (CV) changes during aging can impact disease expression and response to therapy. Aging is associated with increased risk for HTN, MI and HF. Aging-related changes contribute to adverse cardiac remodeling and an accelerated march to HF. The remodeling involves changes in CV structure, cellular and subcellular, pathophysiological pathways, and responses to stress/injury. While optimal healing post injury is critical for survival with favorable outcome, defective post-MI healing with aging contributes to adverse remodeling and poor outcomes. While better post-MI therapies have improved survival, therapy for optimizing post-MI healing is lacking. While early reperfusion therapy may reduce MI size, delayed reperfusion of large MIs may result in reperfusion damage, impaired healing and adverse remodeling in the elderly. Preclinical studies suggest that pathways during healing can be targeted for optimizing post-MI healing and delay the march to HF. Since remodeling and progression to HF in older patients with preserved ejection fraction (HFPEF) or reduced EF (HFREF) are persistent problems, targeting novel pathways for optimizing post-MI healing in HFREF and post-HTN healing in HFPEF holds special promise.

\section{2}

\section{THE ROLE OF ADIPOKINES IN THE REGULATION OF CARDIAC HYPERTROPHY AND REMODELLING}

\section{Karmazyn}

Department of Physiology \& Pharmacology, University of Western Ontario, London, Ontario

Adipocytes represent endocrine organs secreting potent biologically-active molecules called adipokines. Among the best known adipokines primary attention has been directed towards leptin, a $16 \mathrm{kDa}$ protein produced by many tissues including the heart. Leptin acts on specific receptors which are also expressed on the cardiomyocyte. Extensive evidence has shown that leptin exerts a hypertrophic effect via various signalling pathways including RhoA/ ROCK activation. In contrast, the $30 \mathrm{kDa}$ protein adiponectin exerts effects opposite to that of leptin and produces an antihypertrophic effect. Contrary to what is seen with leptin, adiponectin levels are significantly reduced in obese subjects as well as in heart disease including heart failure and increased plasma adiponectin levels are associated with a lower risk of coronary artery disease. Adiponectin plasma concentrations $(3-30 \mu \mathrm{g} / \mathrm{mL})$ are approximately 1000 times higher than leptin and it has been proposed that adiponectin is a critical endogenous cardioprotective and anti-remodelling factor. The "yinyang"-like relationship between leptin and adiponectin suggests that the balance between these two proteins, rather than the absolute values of each, is critical in determining the development of heart disease.

Acknowledgements: Supported by the CIHR.

\section{3}

REMODELING OF CARDIAC ENDOTHELIUM IN PRESSURE OVERLOAD LEFT VENTRICULAR HYPERTROPHY

GS Ajithkumar' ${ }^{1}$ A Vinitha ${ }^{1}$, SS Binilraj ${ }^{1}$, G Sanjay ${ }^{2}$, CC Kartha ${ }^{1}$

${ }^{1}$ Rajiv Gandhi Center for Biotechnology, ${ }^{2}$ Sree Chitra Tirunal Institute for Medical Science \& Technology, India

Cross talk among cardiac endothelial cells and cardiomyocytes could modulate remodeling events in a diseased heart. We analyzed cardiac endothelial cells during evolution of pressure overload cardiac hypertrophy and progression to heart failure in male Wistar rats with constriction of ascending aorta. In echocardiography, left ventricular hypertrophy was seen in rats with aortic constriction at the end of 1 month. Rats were sacrificed at the end of 1, 2, 3, 6 and 12 months after constriction of aorta. After 12 months, in rats with aortic constriction LVEF was reduced and serum BNP levels were $>300 \mathrm{pg} / \mathrm{mL}$. qPCR and immunoblotting revealed increased expression of eNOS, iNOS, ABCG2, ACE $1 \&$ 2, endothelin, VEGFa and VEGFr in microvascular endothelial cells from rats with cardiac hypertrophy. Expression of these molecules was reduced in the endothelial cells of rats with heart failure. Cardiac endothelial cells are altered during cardiac hypertrophy and in failing left ventricle. Increased ABCG2 expression during compensatory cardiac hypertrophy may be an adaptive mechanism of the endothelial cell to counter the deleterious effects of excess superoxide anions during pressure overload.

Funding: Department of Biotechnology, Govrenment of India. 


\section{4}

\section{MICRORNAS AS POTENTIAL THERAPEUTICS IN CARDIOMYOPATHY}

\section{Khullar, A Kumar, S Raut, A Mittal}

Department of Experimental Medicine \& Biotechnology, PGIMER, Chandigarh, India

Cardiomyopathies are heterogeneous group of diseases of the heart muscle characterized by contractile dysfunction in the absence of coronary heart disease, hypertension or congenial heart disease and are important cause of heart failure and associated morbidity and mortality. MicroRNAs are small regulatory RNA molecules which are emerging as important therapeutic targets in cardiovascular diseases including cardiomyopathies. Cardiac hypertrophy and cardiac fibrosis are important features of cardiac remodelling resulting in decline in cardiac function. We examined the expression of microRNAs in dilated and diabetic cardiomyopathies and observed disease specific aberrant expression of several microRNAs in these two different cardiomyopathies. Cardiac expression of anti-hypertrophy miRNA-30c and miRNA-181a was decreased and that of pro-fibrotic microRNAs, mIRNA-21 and miRNA -29a was increased in diabetic cardiomyopathy. Cardiac expression of miRNA-33 was decreased in idiopathic dilated cardiomyopathy. Overexpression and inhibition of these microRNAs with miRNA mimics and antagomirs, in vitro, in cardiomyocytes/fibroblasts showed amelioration of cardiac hypertrophy/cardiac fibrosis, indicating their potential role as novel therapeutic targets in cardiomyopathies.

\section{5}

\section{MITOCHONDRIAL INVOLVEMENT IN CHRONIC HYPOXIA- INDUCED CARDIOPROTECTION}

\section{F Kolar}

Institute of Physiology AS CR, Prague, Czech Republic

It has been well established that living at higher altitude is associated with a decreased mortality from ischemic heart diseases. Experimental studies showed that adaptation to various modes of chronic hypoxia improves cardiac tolerance to injury caused by acute ischemia/reperfusion (I/R) insult. Moreover, chronic hypoxia may have therapeutic effects by attenuating adverse cardiac remodeling and preventing the development of contractile dysfunction after myocardial infarction. Although a significant progress has been achieved in uncovering of a number of factors involved in the chronic hypoxia-induced protection against I/R injury, its complex molecular mechanism still remains poorly understood. The growing body of evidence points to the key role of mitochondrial energy metabolism, ionic transport and signaling functions. As chronic hypoxia seems to utilize essentially the same pool of protective elements as conventional forms of preconditioning, the combination of these two measures fails to provide more efficient temporal cardioprotection than preconditioning alone. However, in contrast to short-lived salutary effects of preconditioning, chronic hypoxia can maintain ischemia-tolerant phenotype for several weeks after the cessation of the stimulus, making its mechanism suitable for potential exploitation.

Funding: Supported by GACR 303/12/1162.

\section{6}

\section{FGF-2 EXERTS ISOFORM-SPECIFIC EFFECTS AGAINST DOXORUBICIN-INDUCED CARDIOTOXICITY}

N Koleini, JJ Santiago, BE Nickel, W Srisakuldee, RR Fandrich, PA Cattini, D Jassal, E Kardami

Departments of Physiology \& Pathophysiology, Human Anatomy \& Cell Sciences, University of Manitoba; Institute of Cardiovascular Sciences, St Boniface Research Centre, Winnipeg, Manitoba

Cardiotoxicity is a main side effect of Doxorubicin (DOX) therapy. FGF-2 is an endogenous cardioprotective agent, although potentially isoform-specific effects in Doxorubicin injury have not been studied. We used FGF-2 transgenic mice to investigate the effect of chronic endogenous expression of FGF-2 isoforms, high (Hi-) or low (Lo-) molecular weight FGF-2, in DOXinduced cardiotoxicity. Mice expressing only Lo-FGF-2 displayed minimal mortality and minimal loss of contractile function assessed at 10 days post DOX by echocardiography. The protective effect of Lo-FGF-2 expression was accompanied by increased accumulation of cardioprotective kinases Akt and PKCepsilon, and downstream targets such as phospho-Connexin43. In vitro, treatment of cardiomyocytes with Hi- (but not Lo-) FGF-2 exacerbated DOX-induced apoptotic cell death. In both in vivo and in vivo models, Hi- and Lo-FGF-2 exerted differential effects on autophagy-associated proteins. We conclude that the beneficial effects of Lo-FGF-2, in contrast to Hi-FGF-2, may be attributed to distinct effects on survival and autophagy.

\section{7}

\section{PDE5 INHIBITION AND PROTECTION OF DIABETIC HEART}

RC Kukreja

Virginia Commonwealth University, Pauley Heart Center, Division of Cardiology, Richmond, Virginia, USA

Phosphodiesterase 5 (PDE5) inhibitors including sildenafil protect against myocardial ischemia/reperfusion (I/R) and ischemic cardiomyopathy. We have also shown that long acting PDE5 inhibitor tadalafil protects type II diabetic heart against I/R injury. Treatment of Leptin receptor null (db/db) diabetic mice with tadalafil $(1 \mathrm{mg} / \mathrm{kg})$ for 28 days significantly reduced fasting glucose, triglycerides and improved LV function. Circulating levels of TNF- $\alpha$, IL- $1 \beta$ and myocardial infarct size following $I / R$ was reduced in tadalafil treated mice compared to control. Tadalafil treated mice had increased plasma NO levels, increased expression of myocardial silent information regulator 1 (SIRT1) as well as PGC1 $\alpha$, a regulator of mitochondrial biogenesis and co-activator of transcription factors impacting energy homeostasis. Tadalafil treatment enhanced phosphorylation of eNOS, Akt, and AMPK in the diabetic hearts. Mitochondrial oxidative phosphorylation with the complex I substrate glutamate was decreased by $50 \%$ in diabetic hearts which was preserved in tadalafil-treated mice. These results demonstrate the ability of tadalafil in protecting diabetic heart against $\mathrm{I} / \mathrm{R}$ injury through mechanisms that blunt inflammation, activate NO, Sirt1/ PGC-1 $\alpha$ signaling preservation of mitochondrial function.

Funding: Supported, in part, by NIH grant HL118808.

\section{8}

\section{CHANGES IN MYOSIN COMPOSITION IN HUMAN END-STAGE FAILING HEART}

\section{Kyselovic}

Comenius University, Faculty of Medicine, Faculty of Pharmacy, Bratislava, Slovak Republic

Myosin is the molecular motor of contraction. In the heart, three distinct myosin heavy chain isoforms coexist in delicate balance. As has been recently shown, the shift from one myosin isoform to other may be one of key factors causing heart failure. In this study we aimed to analyze gene expression of myosin heavy chain isoforms in failing hearts. We examined samples from left ventricles of 40 patients with end-stage heart failure indicated for heart transplantation. We used quantitative RT-PCR to measure mRNA levels of cardiac myosin heavy chain isoforms (MYH6, MYH7, MYH7B), related transcription factors (GATA4, SRF, NKX, YY1) and microRNAs (miR-1, 133a, 208a, 208b, 499, 29b), based on bioinformatic predictions and databases. In adult human failing hearts, we found the slowtwitch myosin heavy chain MYH7 ( 98\%) to be the predominantly expressed isoform whereas fast-twitch MYH6 isoform constitutes just about $1 \%$ of all myosin isoforms. This excessive expression of $\mathrm{MYH} 7$ is regulated by several transcription factors and microRNA, which expression is also altered. Conclusively, dysregulated gene expression of myosin heavy chains resulting in MYH7 upregulation and MYH6 downregulation might be an adaptation in heart failure and interesting target for future pharmacotherapy.

Funding: VEGA 1/0076/2013, VEGA 1/09/2014.

\section{9}

GENDER-RELATED RESPONSE TO ISCHEMIA IN YOUNG SHR RATS EXPOSED TO CROWDING

V Ledvenyiova, I Bernatova ${ }^{1}$, S Carnicka, M Bartekova,

T Ravingerova

Institute for Heart Research, ${ }^{1}$ Institute of Normal and Pathological Physiology, Slovak Academy of Sciences, Centre of Excellence SAS NOREG, Bratislava, Slovak Republic

Gender and social stress are risk factors in etiology of hypertension that impair response to ischemia and reperfusion $(\mathrm{I} / \mathrm{R})$. We explored the impact 
of crowding stress (CS) on effect of I/R in young hypertensive (SHR) male and female rats. Five-week-old SHR and WKY rats were exposed to 2-w CS (livingspace $200 \mathrm{~cm}^{2} / \mathrm{rat}$ and $480 \mathrm{~cm}^{2} /$ rat, resp.). Langendorff-perfused hearts were subjected to 30 -min global ischemia and $2-\mathrm{h}$ reperfusion for the evaluation of ventricular arrhythmias and infarct size (IS). WKY males had higher blood pressure than females, different from opposite sex-specific effects in SHR, while IS and susceptibility to arrhythmias were significantly increased in both sexes. CS-SHR exhibited even higher vulnerability to ventricular tachycardia (VT), but females had shorter VT duration compared to males in this group. In contrast IS was not affected by CS in any of the groups. In addition, CS decreased NOS activity in left ventricle of SHR of both sexes. CS modifies parameters of I/R in a distinct way. Predisposition to hypertension may account for different response to ventricular arrhythmias and lethal injury, which appears to be sex-dependent.

Funding: Grants APVV-0523-10, APVV-0102-11.

\section{0}

\section{THE NEUROPEPTIDE SUBSTANCE P AS A MASTER REGULATOR OF CARDIAC FIBROSIS \\ $\underline{\text { SP Levick }}$}

Department of Pharmacology \& Toxicology, Medical College of Wisconsin, Milwaukee, Wisconsin, USA

We have identified the critical role for the neuropeptide substance P (SP) in the process of cardiac fibrosis. Inhibition of the actions of SP, either by blockade of its receptor (neurokinin-1 receptor) or deletion of the gene encoding substance $\mathrm{P}$ ( $\mathrm{Tacl}$ ) both protect the hypertensive heart from fibrosis, independent of blood pressure. This is not via direct effects of SP on cardiac fibroblasts since while fibroblasts do increase expression of genes related to the extracellular matrix and adhesion in response to SP, this is transient and does not result in phenotypic or functional changes. However, we have identified that SP regulates multiple pathways by which it indirectly causes fibrosis. These include: 1) regulation of cardiac mast cell density and subsequent tryptase production, a pathway that we have previously shown to be important in fibrosis; 2) production of endothelin-1 (ET-1) and regulation of ET-1 receptors on cardiac fibroblasts; and 3) synthesis of IL-6 (which is pro-fibrotic when coupled with its soluble receptor) by cardiac fibroblasts and macrophages. Thus, our recent work has established SP as a master regulator of multiple pathways that are known to play important roles in cardiac fibrosis, and as such has identified an exciting potential target for therapeutic intervention.

\section{1}

\section{A NON-INVASIVE TECHNIQUE FOR TARGETED GENE DELIVERY TO ENHANCE CARDIAC REGENERATION \\ RK Li}

Toronto General Research Institute, University Health Network, University of Toronto, Toronto, Ontario

Following a myocardial infarction (MI), cardiomyocyte loss is associated with a cascade of events, which result in heart failure. Despite developments in clinical management, heart failure remains the major causes of morbidity and mortality following MI. Gene therapy has been demonstrated to enhance cell survival and tissue regeneration, diminish adverse ventricular remodeling and improve cardiac function. However, inefficient gene delivery to the target tissue has limited the potential of this therapy. Ultrasound microbubbles are used clinically for the evaluation of myocardial perfusion. These lipid vesicles can be carriers for the targeted gene delivery. Ultrasound-targeted microbubble destruction (UTMD) can provide minimally invasive, repeatable, and targeted gene delivery to the infarcted myocardium to restore ventricular function. We hypothesized that a novel UTMD strategy would induce tissue regeneration, stem cell homing and improve ventricular function after MI. We have evaluated optimal time and conditions, as well as the optimal microbubble, for target gene delivery. The UTMD successfully delivered the genes to the infarct after MI, induced angiogenesis, and enhanced cardiac repair and function. UTMD is an ideal method for gene delivery to enhance heart regeneration after $\mathrm{MI}$.
072

\section{ATHEROSCLEROSIS AND REMODELING OF THE ULNAR ARTERY AFTER REMOVAL OF THE RADIAL ARTERY FOR USE IN CORONARY ARTERY BYPASS GRAFTING - A PROSPECTIVE STUDY COMPARING ULTRASOUND- DOPPLER}

ML Lima, LN Lougon, FJ dos Reis, FOG Teixeira, WJ Gomes Hospital Meridional-Serviço de Cirurgia Cardiovascular, Cariacica, Brazil

To comparatively analyze arterial remodeling and accelerated atherosclerosis in the ulnar artery of the same donor arm of patients undergoing radial artery harvesting for use in CABG with Doppler ultrasound in the pre- and postoperative period.

METHODS: A prospective comparative study in 99 right-hand patients, undergoing harvesting of left radial artery. The variables included: peak systolic velocity, end-diastolic velocity, average velocity in the mean time, resistance index, intima-media thickness, diameter, area, peak systolic flow and average flow.

RESULTS: In the ulnar artery there was a statistically significant increase in the post-operative in relation to the pre-operative period of the following variables: diameter $(3.85 \pm 0.87$ vs $4.49 \pm 0.69 \mathrm{~mm} ; \mathrm{P}=0.0002)$, area $\left(0.12 \pm 0.05 \mathrm{vs} 0.16 \pm 0.05 \mathrm{~cm}^{2} ; \mathrm{P}=0.0001\right)$, peak systolic flow $(357.02 \pm 181.91$ vs $555.16 \pm 228.2 \mathrm{~mL} / \mathrm{min} ; \mathrm{P}=0.0003)$ and average flow $(59.82 \pm 34.2$ vs $105.08 \pm 108.23 \mathrm{~mL} / \mathrm{min} ; \mathrm{P}=0.0004)$. Intima-media thickness measured in the ulnar artery did not show a statistically significant difference $(\mathrm{P}=0.24)$. CONCLUSION: The chronic increase flow in ulnar artery did not accelerated atherosclerosis in the postoperative period after radial artery harvesting in CABG.

\section{3}

METABOLIC STRESS TRIGGERS A NOVEL SURVIVAL PATHWAY LINKED TO ALTERNATIVE SPLICING OF BNIP3

ЦLin, H Gang, LA Kirshenbaum

Institute of Cardiovascular Sciences St Boniface General Hospital Research Centre, Winnipeg, Manitoba

Programmed cell death is a central feature of myocardial infarction and heart failure but the inciting mechanisms are poorly understood. The hypoxia-inducible death protein Bnip3 is a critical component of the intrinsic apoptosis death pathway that can also drive autophagy but the mechanisms that differentially regulate these divergent actions remain cryptic. Here we show that exclusion of exon3 from Bnip3 mRNA (Bnip3 3 ex3) by alternative splicing is an obligatory step that converts Bnip3 from cell death to cell growth in cardiac muscle cells. Conditions that favored Bnip3 exon3 skipping inhibited apoptosis and autophagy while inducing gene transcription for cardiac hypertrophy and mitochondrial biogenesis. Conversely, inhibition of Bnip3Dex3 triggered mitochondrial perturbations and death during hypoxia. Hence alternative splicing of Bnip3 mRNA highlights a novel unrecognized survival mechanism that opposes mitochondrial defects and cell death of ventricular myocytes during hypoxic stress.

\section{4}

STIMULATING CARDIAC GLUCOSE OXIDATION AS AN APPROACH TO TREAT HEART FAILURE IN OBESITY

\section{GD Lopaschuk, S Sankaralingam}

University of Alberta, Edmonton, Alberta

Obesity increases heart failure incidence. Altered cardiac energy metabolism is a potential contributor to heart failure in obesity, including increased cardiac fatty acid oxidation that can decrease cardiac efficiency and heart function. We examined what effect decreasing body weight in obese mice with heart failure has on cardiac function and energy metabolism. Obesity and heart failure were induced in mice by feeding them a high fat (HF) diet and then producing a transverse aortic constriction (TAC). Obese heart failure mice were either: 1) maintained on a HF diet for 10 weeks; 2) caloric restricted (40\% decrease in food intake); or 3) switched to a low fat (LF) diet. Obese heart failure mice developed systolic dysfunction, which was improved by weight loss due to caloric restriction or a LF diet. Obese mouse hearts had increased reliance on fatty acid 
oxidation as a source of energy (94\%) compared to lean controls (46\%). Weight loss due either to caloric restriction or a LF diet decreased the contribution of fatty acid oxidation to energy production (from $94 \%$ to $51 \%$ and $48 \%$, respectively), which was accompanied by an increase in glucose oxidation, and an improved cardiac work and efficiency. Therefore, weight loss in obese mice with heart failure improves cardiac glucose oxidation, and improves cardiac function.

\section{5}

\section{2-METHOXYESTRADIOL: A GENDER SPECIFIC THERAPY AGAINST CELLULAR REJECTION IN CARDIAC TRANSPLANTATION}

JGY Luc, R Paulin, JY Zhao, DH Freed, ED Michelakis, J Nagendran University of Alberta, Edmonton, Alberta

BACKGROUND: 2-Methoxyestradiol (2ME2) is an endogenous metabolite of estrogen previously studied as a selective antimitotic-agent against proliferative cancer cells. As its effect in a transplant rejection setting remains unknown, we hypothesized that $2 \mathrm{ME2}$ can inhibit activated T-cell function.

METHODS: Human T-cells were cultured and pre-treated with 2ME2 overnight before activation. ELISA, western immunoblotting, Annexin V and proliferation assays were performed. Results: Cytokine levels in 2ME2 treated activated T-cells were not significantly reduced whereas Tcell proliferation was significantly blunted, with an observed $10 \%$ decrease in apoptosis, a $30 \%$ decrease in caspase- 9 activity without an increase in toxicity. The stress-induced senescent marker p16 was completely ablated upon 2ME2 treatment.

CONCLUSIONS: Our study is the first to show that 2ME2 is able to effect immunosuppression causing activated T-cells to not proliferate, not apoptose and not be harmfully stressed through a senescent pathway. Baseline production of cytokines show that the T-cells remain healthy where they are needed in the innate response. Therefore, 2ME2 may lead to an oral immunomodulatory adjunctive therapy for individuals undergoing cardiac transplantation.

\section{6}

UTERINE IMMUNOPRIVILEGED STEM CELLS FOR CARDIAC REGENERATION AFTER MYOCARDIAL INFARCTION

\section{A Ludke, J Wu, SH Li, RD Weisel, RK Li}

\section{Toronto General Research Institute, Toronto, Ontario}

Premenopausal women are more protected than men from cardiovascular disease. Our previous study showed that uterine stem cells home to the myocardium after myocardial infarction (MI) and are involved in neovascularization. In this study we focused in the identification of immnoprivileged uterine stem cells for cardiac regeneration. When compared to bone marrow, the uterus had the highest percentage $(20 \%)$ of cells expressing low levels of the Major Histocompatibility Complex I (MHC I) measured by flow cytometry. The uterine MHC I (low) population also expressed other stem cell markers, formed colonies and differentiated. In an allogeneic mixed leukocyte co-culture system, MHC I ${ }^{\text {(low) }}$ uterine cells were comparable to mouse mesenchymal stem cells (MSCs) in resisting cell death and inhibiting leukocyte proliferation. The angiogenic capacity of these cells was shown in vitro and in vivo and was significantly greater than MSCs. Intramyocardial injection of allogeneic $\mathrm{GFP}^{+} \mathrm{MHC}$ $\mathrm{I}^{\text {(low) }}$ cells in a murine model of MI was shown to engraft in cardiac tissue and restore cardiac function evaluated by echocardiography. This study identified immunoprivileged uterine stem cells which have great potential to induce neovascularization and could be used as allogeneic cell therapy for cardiac regeneration.

\section{7}

EFFECT OF TRANS-FATS ON CARDIOMYOCYTE CA ${ }^{2+}$ TRANSIENTS AND CELL SIGNALING WITH SIMULATED ISCHEMIA/REPERFUSION

R Ganguly, IG Maddaford, GN Pierce

Institute of Cardiovascular Sciences, and CCARM, St Boniface

Hospital, and the University of Manitoba, Winnipeg, Manitoba

Ruminant trans-fats (rTFA) and industrial trans-fats (iTFA) have different effects on heart disease. We administered rTFA vs. iTFA for $24 \mathrm{~h}$ to isolated cardiomyocytes and measured the effects on cardiomyocyte cell signaling during simulated ischemia/reperfusion (IR). Cells were exposed to simulated ischemia for $60 \mathrm{~min}$ and calcium transients were measured. Cardiomyocytes exposed for $24 \mathrm{~h}$ to $400 \mu \mathrm{M}$ elaidic acid (EA), an iTFA, induced a continual rise in diastolic $\mathrm{Ca}^{2+}$ levels, although $\mathrm{Ca}^{2+}$ transients did not change. This rise was significantly greater than that exhibited by cells exposed to vaccenic acid (VA), an rTFA, and was qualitatively similar during $60 \mathrm{~min}$ of reperfusion. EA pre-treatment increased cleaved caspase- 3 activity in both the ischemia and IR conditions. An increase in TUNEL staining in IR with EA pre-treatment further supported this data. The number of dead cells increased significantly after IR in cells pretreated with EA. VA had no effect on $\mathrm{Ca}^{2+}$ transients or apoptotic signaling proteins compared to control. EA increased death with or without IR injury. VA had no effect on cell death or $\mathrm{Ca}^{2+}$ transients compared to control.

Funding: Supported by the Heart and Stroke Foundation of Canada and CIHR.

\section{8}

\section{FOXO1 SIGNALING IN CARDIOVASCULAR DISEASE DURING CALORIC RESTRICTION}

\section{N Makino}

\section{Kyushu University, Beppu, Japan}

This study examined whether the forkhead transcription factors of $\mathrm{O}$ group 1 (FoxO1) might be involved in telomere biology and cardiac remodeling during $\mathrm{CR}$. We used FoxO1-knockout heterozygous mice (HT) in which the level of Foxo1 mRNA was reduced by $50 \%$ or more, compared to wild-type mice (WT). Both WT and HT were fed ad libitum (AL) or $30 \%$ of the AL diet for CR for 15 weeks. Telomere (TL) size in heart tissues was significantly lower in the HT groups than the WT groups, and TL attrition was not observed in either WT-CR or HT-CR. Telomerase activity was elevated to a greater extent in WT-CR than WT-AL, but was not significantly different between the HT groups. Cardiac autophagy, which was evaluated by light chain 3II (LC3II) protein expression and immunostaining, was enhanced only in WT-CR. No significant differences in echocardiac parameters were observed between the two HT groups. The present study showed that CR activated the telomerase enzyme and affected cardiac autophagy in WT, but had no discernible effects in HT. Therefore, FoxO1 signaling may play an important role in the maintenance of telomere biology and may be associated with the protection against cardiovascular disease.

\section{9}

\section{LYSOPHOSPHATIDIC ACID INDUCES STEM CELL DIFFERENTIATION INTO CARDIOMYOCYTES}

\section{G Maan, S Thakur, AR Baydoun}

School of Life \& Medical Sciences, University of Hertfordshire, Hertfordshire, United Kingdom

INTRODUCTION: Generation of cardiomyocytes (CMs) from stem cells (SCs) has often been achieved using chemical agents with limited clinical benefits. In this study we have investigated whether differentiation could be induced in the P19 mouse teratocarcinoma SC line by the endogenous biolipid, lysophosphatadic acid (LPA).

METHODS: P19 SCs maintained in routine culture in supplemented $\alpha$-MEM were aggregated into embryoid bodies in the presence of LPA $(0-20 \mu \mathrm{M})$ or $1 \%$ dimethyl sulfoxide (DMSO) for 4 days and plated in a monolayer for a further 12 days. Lysates collected on days 6 and 12 were subjected to Western blotting for cardiac specific myosin light chain-1v protein $(\mathrm{MLC1}$ ) .

RESULTS: LPA induced a concentration- and time-dependent differentiation of P19 cells into CMs. MLC1v expression peaked significantly above basal on day $6(\mathrm{P}<0.001)$ with $5 \mu \mathrm{M}$ LPA and beating clusters were observed with $20 \mu \mathrm{M}$ LPA on day 7 after plating cells. These effects were comparable to those of $1 \%$ DMSO.

CONCLUSION: LPA induces P19 SC differentiation into CMs as efficiently as $1 \%$ DMSO and may prove to be a physiological molecule with clinical potential for cardiac regeneration through stem cell therapy. We 
aim to further investigate the signalling mechanisms that mediate the actions of LPA in SC differentiation.

\section{0 \\ GENOMIC AND NON-GENOMIC ROLES FOR CLASS I HDACS IN HEART FAILURE}

\section{TA McKinsey}

University of Colorado, Denver, Colorado, USA

Efficacy of small molecule histone deacetylase (HDAC) inhibitors has been demonstrated in animal models of heart failure. While mounting evidence suggests an application for HDAC inhibitors for the treatment of heart failure, the mechanisms by which HDACs control cardiac hypertrophy and fibrosis remains unclear. Furthermore, since the pharmacological inhibitors used in the pre-clinical heart failure studies target 11 distinct HDAC enzymes, the identity of the HDAC isoforms that control cardiac remodeling remains unknown. Our recent data demonstrate novel roles for a subset of HDACs, class I HDACs $-1,-2$ and -3 , in the control cardiac hypertrophy and fibrosis through their effects on mitogen activated protein kinase signaling. Class I HDAC inhibitors block signal-dependent activation of ERK1/2 and JNK in cardiac cells without affecting p38. Suppression of ERK1/2 signaling by class I HDAC inhibitors involves induction of an ERK-specific phosphatase, DUSP5. In contrast, class I HDAC inhibition appears to block cardiac JNK signaling by promoting acetylation and inactivation of an upstream kinase(s). These findings define novel genomic and non-genomic roles for class I HDACs in the control of signaling events that culminate in heart failure.

Funding: Supported by NIH grants HL116848 and AG043822

\section{1}

OXIDATIVE STRESS AND INFLAMMATION IN ATHEROSCLEROSIS: ROLE OF LOX-1, MITOCHONDRIAL DNA DAMAGE, AUTOPHAGY AND NLRP3 INFLAMMASOME LL Mehta, X Wang, Z Ding

University of Arkansas for Medical Sciences, Little Rock, Arkansas, USA

Alterations in innate immunity is a key component of atherosclerosis. NLRP3 inflammasome activation participates in innate immunity and induces release of IL-1 $\beta$ and IL-18. We hypothesized that damaged mitochondrial DNA (mtDNA) that escapes from autophagy could trigger NLRP3 inflammasome, and LOX-1 may play a critical role in this process. We studied and found intense autophagy, TLR9 expression and inflammatory signals in the aortas of LDLr null mice fed high fat diet. Deletion of LOX-1 attenuated autophagy, TLR9 expression as well as inflammation. Damaged mtDNA signal was also high in LDLr null mice, and this signal was attenuated by LOX-1 deletion. Thus, it appears that oxidative stressmediated damaged mtDNA that escapes autophagy induces a potent inflammatory response. Next, the link between LOX-1, autophagy, myDNA damage and inflammation was studied in THP1 macrophages exposed to LPS. We observed that LOX-1 mediated autophagy and mtDNA damage play an essential role in NLRP3 inflammasome release during inflammation, and links LOX-1 with mtDNA damage and NLRP3 inflammasome activation. Of note, Duewell et al (Nature 2010) have shown that NLRP3 inflammasomes are required for atherogenesis and activated by cholesterol crystals that form early in disease.

\section{2}

\section{DISENTANGLING MECHANISMS OF TRANSCRIPTIONAL REGULATION IN AORTIC VALVE STENOSIS}

\section{JD Miller}

\section{Department of Surgery and Department of Physiology \&} Biomedical Engineering, Mayo Clinic, Rochester, Minnesota, USA

Hemodynamically significant aortic valve stenosis affects $3 \%$ of the population over age 65 , and portends a 5 -year event-free survival of less than $40 \%$. While surgical or transcatheter procedures remain the only available treatment for advanced aortic valve stenosis, recent work showing that aortic valve calcification is an active process involving osteoblast-like cells suggests that valve calcification may be a modifiable, druggable process. Our laboratory has focused on mechanisms that contribute to genomic instability and dysregulation of osteogenic gene expression in aortic valves with aging. Most recently, our program has had a particular emphasis on epigenetic regulation of gene expression in calcifying valves. More specifically, here we present work suggesting that changes in histone acetylation and DNA methylation are major contributors to pathophysiological gene expression in calcifying aortic valves, and may represent viable therapeutic targets to slow progression of valve calcification and progression to severe aortic valve stenosis.

\section{3}

CARDIOPROTECTIVE ROLE OF MIR-133A IN THE DIABETIC HEARTS

PK Mishra

Cellular \& Integrative Physiology, University of Nebraska Medical Center, Omaha, Nebraska, USA

Autophagy is ubiquitous in all forms of heart failure and has dual (protective or detrimental) effects. One of the intriguing questions is how the basic level of cardiac autophagy is maintained and autophagy flux is regulated in the heart. To address this question and to investigate the role of miR-133a in regulation of autophagy, Ins2+/- Akita mice (spontaneous, chronic, T1D model relevant to humans) were used, where miR-133a is down regulated and autophagy is up regulated. We over expressed miR-133a in Akita and WT hearts, and measured the signaling molecules involved in regulation of autophagy: AMPK, mTOR, Beclin1 and LC3B. The results revealed that supply of miR-133a mimic restores the cardiac levels of the above signaling molecules in Akita. To understand the underlying mechanism, HL1 cardiomyocytes were treated with high glucose and miR-133a and levels of autophagy signaling molecules were assessed. The results were similar to that of Akita. The specific role of miR-133a in autophagosome formation and their degradation was determined by treating HL1 cells with RFP-LC3 and monodansylcadaverin (MDC), respectively. The results show that miR-133a normalizes glucose mediated induction of autophagosomes and their subsequent degradation. Based on these findings, it is concluded that miR-133a restores autophagy homeostasis in diabetic hearts.

Funding: Supported by NIH grants HL-113281 and HL-116205.

\section{4}

PROBING THE NANO-DOMAINS OF CARDIAC $\mathrm{CA}^{2+}$ SIGNALING WITH GENETICALLY TARGETED BIOSENSORS M Morad, S Phlavan, XH Zhang

Cardiac Signaling Center of University of South Carolina, Medical University of South Carolina, and Clemson University, Charleston, South Carolina, USA

It is now recognized that mutations in $\mathrm{Ca}^{2+}$ signaling proteins can cause cardiovascular pathologies. Genetically targeted biosensors make it possible to probe the nano-domains of $\mathrm{Ca}^{2+}$ signaling proteins. We engineered probes targeted to ryanodine receptor (RyR2, FKBP-GCamP6), Phospholamban (PLB, PLB-Cam), and mitochondrial cytochrome C (Mitycam-E31Q) and monitored their signals in voltage-clamped rat cardiomyocytes. 2-D confocal and TIRF imaging of adult cardiomyocytes showed sarcomeric images for FKBP-GCamP6, veil-like pattern and nuclear-envelope staining for PLB-Cam, and punctate longitudinal pattern for mitycam-E31Q. TIRF-imaging with FKBP-GCamP6 showed brief $\mathrm{Ca}^{2+}$ sparks confined to the $z$-lines, preceded by smaller 'sparklets', decreased spark frequency and size and bell-shaped voltage dependent Cai-transients, consistent with the probe's ability to detect $\mathrm{Ca}^{2+}$ released at the nanodomains of RyR2. Mitycam-E31Q signals were confined to mitochondria and showed diverse patterns of $\mathrm{Ca}^{2+}$ release and uptake during spontaneous pacing of neonatal cardiomyocytes or on caffeine-induced $\mathrm{Ca}^{2+}$ release, suggesting that mitochondrial $\mathrm{Ca}^{2+}$ release and uptake plays a critical role in regulating spontaneous pacing in developing cardiomyocytes. Funding: Supported by NIH grant RO-1 HL16152 \& HL107600. 


\section{5}

BENDAVIA (MTP-131), A MITOCHONDRIA-TARGETING PEPTIDE, IMPROVES ADP-STIMULATED MITOCHONDRIAL RESPIRATION IN CARDIOMYOCYTES OF DOGS WITH CHRONIC HEART FAILURE

PM Mohyi, RC Gupta, HN Sabbah

Henry Ford Hospital, Detroit, Michigan, USA

BACKGROUND: Mitochondria (MITO) in heart failure (HF) have abnormal respiration leading to reduced ATP synthesis that adversely impacts LV function. Therapy with Bendavia (BEN, MTP-131) improves LV function, reverses MITO abnormalities \& normalizes MITO ATP synthesis in HF dogs. This study examined the effects of BEN on MITO ADP. stimulated state- 3 respiration in cardiomyocytes isolated from dogs with microembolization-induced HF.

METHODS \& RESULTS: A collagenase-based enzymatic process was used for cardiomyocyte isolation. Equal aliquots of cardiomyocytes were incubated in $0 \mu \mathrm{M}, 0.01 \mu \mathrm{M}, 0.10 \mu \mathrm{M}, 1.0 \mu \mathrm{M}$ and $10 \mu \mathrm{M}$ concentration of BEN for one hour at $37^{\circ} \mathrm{C}$. At the end of incubation, ADP-stimulated state-3 respiration was measured using a Strathkelvin respirometer and quantified in nAtom Oxygen/min/mg protein. Compared to baseline state-3 respiration (248 \pm 9 ), incubation of failing cardiomyocytes with BEN significantly increased respiration to $303 \pm 33$ at $0.01 \mu \mathrm{M}, \mathrm{P}<0.05$; $405 \pm 39$ at $0.10 \mu \mathrm{M}, \mathrm{P}<0.05 ; 371 \pm 28$ at $1.0 \mu \mathrm{M}, \mathrm{P}<0.05$; and $346 \pm 29$ at $10.0 \mu \mathrm{M}, \mathrm{P}<0.05$.

CONCLUSIONS: The results indicate that the effects of BEN on MITO respiration in cardiomyocytes is direct and that the improvement in respiration can occur as early as one hour after therapy with BEN.

\section{6}

THE EFFECT OF EXTRACELLULAR MATRIX STIFFNESS ON HUMAN BONE MARROW-DERIVED MESENCHYMAL PROGENITOR CELL DIFFERENTIATION

AL Müller ${ }^{1}$, Y Li² $^{2}$, B Hinz ${ }^{3}$, DH Freed ${ }^{1}$

${ }^{1}$ Department of Physiology, University of Alberta, Edmonton, Alberta; ${ }^{2}$ St Boniface Research Centre, University of Manitoba, Winnipeg, Manitoba; ${ }^{3}$ Matrix Biology Group, University of Toronto, Toronto, Ontario

Cell fate of human mesenchymal progenitor cells (hMPCs) are influenced by the surrounding microenvironment, including surface tension stiffness. It is important to determine how this factor could influence hMPCs to endogenously differentiate into a pro-fibrotic phenotype as bone marrow-derived cells contribute to pathological fibrosis in patients with cardiovascular disease. HMPCs were isolated from the bone marrow of patients undergoing cardiac surgery and cultured on fibronectin-coated plates with surface tension pressures of $2 \mathrm{kPa}$, simulating bone marrow; $15 \mathrm{kPa}$, simulating healthy left ventricle; and $100 \mathrm{kPa}$, simulating a fibrotic scar.

We found that softer surface tensions (both $2 \mathrm{kPa}$ and $15 \mathrm{kPa}$ ) promoted a non-pro-fibrotic phenotype of hMPCs by decreasing the protein quantity of non-muscle myosins, ED-A fibronectin, and mature collagen-1. Interestingly, at $2 \mathrm{kPa}$, high mRNA levels of collagen-1 and non-musclemyosins declined at $15 \mathrm{kPa}$, indicating translational regulation of these proteins in the bone marrow environment. Acknowledgments: Grant funding provided by CIHR and St Boniface Research Foundation.

\section{7}

\section{MONOAMINE OXIDASES AS NOVEL SOURCES OF OXIDATIVE STRESS IN DIABETES: A TRANSLATIONAL APPROACH}

\section{Muntean}

Department of Pathophysiology, University of Medicine and Pharmacy of Timisoara, Romania

Monoamine oxidases (MAOs) are mitochondrial dehydrogenases with two isoforms (A and B) that catalyze the electron transfer from biogenic amines to molecular oxygen via a reaction that generates hydrogen peroxide $\left(\mathrm{H}_{2} \mathrm{O}_{2}\right)$ as by-product. We have previously reported an increased expression of both MAO isoforms in murine models associated with vascular inflammation (induced by LPS) and hypertension (after Ang II treatment) that elicited subsequent endothelial dysfunction. We further hypothesized that MAO-derived $\mathrm{H}_{2} \mathrm{O}_{2}$ contributes to vascular impairment in diabetes and enzyme inhibition could alleviate it. The effects of MAO inhibitors on endothelium-dependent relaxation (EDR) were studied in aortic segments isolated from rats with streptozotocin-induced diabetes and mammary arteries harvested from diabetic patients undergoing the revascularization procedure. An important decrease in EDR and increase in $\mathrm{H}_{2} \mathrm{O}_{2}$ generation were found. Also, MAO-A and B expression was increased in both murine and human diabetic rings. Incubation of vascular segments with MAO inhibitors attenuated $\mathrm{H}_{2} \mathrm{O}_{2}$ production and partly restored EDR. Monoamine oxidases are novel contributors to endothelial dysfunction in diabetes.

Funding: Provided by internal grants PII-C2-TC-2014 \& PIII-C1-PCFI-2014/2015.

\section{8}

CURRENT IMPACT OF THE IMPLANTABLE DEFIBRILLATOR ON PRIMARY PREVENTION OF SUDDEN DEATH

A Uy-Evanadoy ${ }^{1}$, K Narayanan ${ }^{1}, K_{\text {Keinier }}{ }^{1}$, C Teodorescu', H Chugh', E Marijon', K Gunson'2, J Jui'², SS Chugh'

${ }^{1}$ Cedars-Sinai Medical Center Los Angeles, Los Angeles, California; ${ }^{2}$ Oregon Health \& Science University, Portland, Oregon, USA

Randomized trials have established the benefit of the implantable cardioverter defibrillator (ICD) for primary prevention of sudden cardiac death (SCD) in subjects with severe LV dysfunction. However, only a minority of SCD subjects in the general population have low EF. Further, studies have suggested that ICDs are significantly underutilized in the community even among those who are eligible. In the Oregon Sudden Unexpected Death Study, we undertook a systematic investigation of the prevalence rates and influencing factors for deployment of the primary prevention ICD among subjects who eventually suffered SCD in the community. Less than a quarter of the SCD victims had a screening echocardiogram prior to arrest; among those with echo, only $20 \%$ were guideline eligible for primary ICD, $\&$ finally out of those eligible, only $13 \%$ were actually implanted. While older age and comorbidity may explain non-implantation in some, additional factors such as socioeconomic factors and practice patterns clearly warrant further investigation. Our data highlight the need for further systematic efforts to optimize primary ICD use in the general population.

089

QRS FRAGMENTATION ON THE 12-LEAD ELECTROCARDIOGRAM AS A RISK MARKER FOR SUDDEN CARDIAC ARREST IN THE GENERAL POPULATION

K Narayanan ${ }^{1}$, L Zhang1 ' C Kim ${ }^{1}$, A Uy-Evanado ${ }^{1}$, C Teodorescu ${ }^{1}$, K Reinier1', H Chugh1', K Gunson'2, J Jui' ${ }^{2}$, SS Chugh'1

${ }^{1}$ Cedars Sinai Medical Center, Los Angeles, California; ${ }^{2}$ Oregon Health and Science University, Portland, Oregon, USA

We investigated the utility of QRS fragmentation as a risk marker for sudden cardiac arrest (SCA) in the general population. From a prospective, population-based study of SCA (population approx. 1 million), SCA cases were compared with geographic controls. QRS fragmentation was identified in a blinded manner from ECGs prior \& unrelated to the SCA event $\&$ defined as described in literature. Cases $(n=475) \&$ controls $(n=634)$ were similar with respect to age, proportion of males, BMI and prior myocardial infarction; cases were more likely to have severe LV dysfunction and diabetes. Overall fragmentation $(29.5 \%$ vs. $26.0 \% ; \mathrm{P}=0.20)$, as well as inferior $(21.1 \%$ vs. $21.1 \% ; \mathrm{P}=0.97)$ and anterior fragmentation $(9.5 \%$ vs. $7.4 \% ; \mathrm{P}=0.22)$ was similar between cases and controls; however, lateral fragmentation $(6.9 \%$ vs. $2.8 \% ; \mathrm{P}<0.01)$ was observed significantly more in cases. In multivariable analysis, lateral fragmentation (OR 2.5 [95\% CI 1.1 to 5.6]; $\mathrm{P}=0.02$ ) was an independent predictor of SCA. Lateral territory fragmentation appears to be a risk marker associated with SCA in the general population. 


\section{0}

WHO GETS THE HEART ATTACK? FROM BENCH TO BEDSIDE, AND FROM MUMMIES TO POPULATION...

\section{¿Narula}

The Zena and Michael A Wiener Cardiovascular Institute, Icahn School of Medicine at Mount Sinai, New York, USA

More often than not, acute coronary events including heart attacks and sudden cardiac death are the first manifestation of coronary artery disease in otherwise completely healthy and asymptomatic subjects. It is well established that the narrowing of the coronary vasculature is not the determinant of adverse outcomes and we need to think outside the lumen. The coronary lesions that lead to acute events carry a distinct morphological signature. What approach should we develop for a worried well? Are the plaque characteristics amenable to noninvasive or invasive imaging? Can imaging data be applied to high-risk individuals or can it be used for the population at large? When did we start suffering from this scourge? Is coronary disease an ailment of modern man or industrialization? Could imaging of 4000-year old mummies shed light on the evolution of disease? Can we exploit the knowledge to prevent unfortunate events? Lets stitch the evolutionary, historical and modern data together to promote the cardiovascular health globally.

\section{1}

HIF-1A ON INFARCT SIZE-LIMITING EFFECT OF POSTCONDITIONING AFFORDED BY EPOXYEICOSATRIENOIC ACID ANALOG IN RAT HEARTS

J Neckárir ${ }^{1}$, A Hsu' ${ }^{1}$, MAH Khan ${ }^{1}$, GJ Gross ${ }^{1}$, JR Falck², JD Imig' ${ }^{1}$ Medical College of Wisconsin, Milwaukee, Wisconsin; ${ }^{2}$ University of Texas Southwestern, Dallas, Texas, USA

The infarct size-limiting action of a novel epoxyeicosatrienoic acid (EET) analog (EET-B) in reperfusion (postconditioning) and the contribution of hypoxia inducible factor- $1 \alpha$ (HIF-1 $\alpha$ ) were investigated. Adult male SD rats were subjected to $30 \mathrm{~min}$ left coronary artery occlusion and $2 \mathrm{~h}$ of reperfusion. The endogenous EET, 14,15-EET or analog EET-B (both $2.5 \mathrm{mg} / \mathrm{kg}$ ) administered i.v. $5 \mathrm{~min}$ before reperfusion reduced infarct size expressed as a percentage of the area at risk (AR) by $29 \%$ and $33 \%$. While EET antagonist 14,15-EEZE $(2.5 \mathrm{mg} / \mathrm{kg})$ and HIF- $1 \alpha$ inhibitors, 2-methoxyestradiol $(2.5 \mathrm{mg} / \mathrm{kg})$ and acriflavine $(2 \mathrm{mg} / \mathrm{kg})$ completely abolished the protective effect of EET-B. In immunohistology, HIF-1 $\alpha$ signal markedly increased in the AR compared to non-ischemic septum at the end of ischemia $(9.3 \pm 1.1$ vs $0.3 \pm 0.1 \%)$. After $20 \mathrm{~min}$ and $2 \mathrm{~h}$ of reperfusion, HIF- $1 \alpha$ immunoreactivity in AR markedly decreased to $2.4 \pm 0.5 \%$ and $1.9 \pm 0.4 \%$, respectively in the controls. EET-B administration blunted the decrease of HIF-1 $\alpha$ immunoreactivity in ischemized tissue. In conclusion, EET-B provides postconditioning protection against myocardial infarction in rats. We suggest that increased HIF-1 $\alpha$ level plays an important role in this cardioprotective mechanism.

\section{2}

\section{ADAPTATION TO CHRONIC HYPOXIA IMPROVES CARDIAC ISCHEMIC TOLERANCE IN SPONTANEOUSLY HYPERTENSIVE RATS}

LNeckar ${ }^{1}$, I Brabcova ${ }^{2}$, R Weissova ${ }^{2}$, P Zajickova ${ }^{1}$, P Mandikova ${ }^{1}$, J Silhavy ${ }^{1}$, J Zurmanova ${ }^{2}$, O Novakova ${ }^{2}$, M Kalous ${ }^{2}$, M Pravenec ${ }^{1}$, F Kolar $^{1}$

${ }^{1}$ Institute of Physiology, Academy of Science; ${ }^{2}$ Faculty of Science, Charles University, Prague, Czech Republic

The effect of adaptation to chronic continuous hypoxia $(\mathrm{CCH})$ on ischemia/reperfusion $(\mathrm{I} / \mathrm{R})$ injury and the expression of selected cardioprotective proteins and signaling molecules were analysed in left ventricles of spontaneously hypertensive rats (SHR) and in a novel conplastic strain SHR- $m t^{B N}$. These rats are characterized by a selective replacement of the mitochondrial genome of SHR with that of Brown Norway strain, which is more tolerant to ischemic injury. Rats were kept 3 weeks at $\mathrm{CCH}$ (inspired $\mathrm{O}_{2}$ fraction 0.1 ). $\mathrm{CCH}$ reduced myocardial infarct size from $71 \pm 4 \%$ of the area at risk in normoxic to $49 \pm 6 \%$ in hypoxic SHR. It was accompanied by decreased mRNA levels of thioredoxin enzymes, protein level of complex IV (C IV) and the cytosolic superoxide dismutase and increased mRNA levels of catalase, hexokinase 2 (HK2) and hypoxia inducible factor $1 \alpha$ (HIF-1 $\alpha$ ). As compared to SHR, CCH induced more pronounced infarct size-limiting effect (by 19\%), elevated HK2 and HIF-1 $\alpha$ mRNA levels and reduced C IV protein in SHR-mt ${ }^{\mathrm{BN}}$. Overall, $\mathrm{CCH}$ protects SHR hearts against acute $\mathrm{I} / \mathrm{R}$ injury and mitochondrial genes can contribute to this protective effect.

\section{3}

\section{MECHANISMS OF HEART ATTACK AND STROKE: CRITICAL ROLES OF PLATELETS IN ATHEROTHROMBOSIS $\underline{\mathrm{H} \mathrm{Ni}}$}

Department of Laboratory Medicine and Pathobiology, University of Toronto; and Canadian Blood Services, Toronto, Ontario

Platelets are anucleate cells generated from bone marrow. After being released into the blood, platelets play essential roles in hemostasis. However, the processes of platelet adhesion and aggregation at the site of vascular injury may also cause vessel occlusion, and lead to thrombotic diseases. The formation of platelet rich thrombi following the rupture of atherosclerotic lesions is a common cause of myocardial and cerebral infarction. Most recently, the roles of platelets in the initiation and development of atherosclerotic lesions are also highlighted. It has been documented for $\sim 5$ decades that fibrinogen $(\mathrm{Fg})$ is required for platelet aggregation. However, using intravital microscopy thrombosis models, we found that platelet aggregation and thrombus formation occurred in mice lacking Fg and/or both Fg and VWF. We further demonstrated that Fg/ VWF-independent platelet aggregation can be induced in vitro under more physiological conditions (i.e. non-anticoagulated blood) and beta3 integrin is the platelet receptor required for the aggregation. In this presentation, the roles of fibronectin and other novel beta 3 integrin ligands in atherothrombosis will be discussed.

Funding: Provided by CIHR, CFI, HSF of Ontario and Canadian Blood Services.

\section{4}

PHOSPHORYLATION OF MITOCHONDRIAL CA ${ }^{2+}$ UNIPORTER REGULATES MITOCHONDRIAL $\mathrm{CA}^{2+}$ UPTAKE AND APOPTOTIC CELL DEATH IN CARDIOMYOCYTES LO-Uchi' ${ }^{1}$, GL Smith ${ }^{2}$, RT Dirksen ${ }^{3}$, W Wang ${ }^{4}$, R Rizzuto ${ }^{5}$, SS Sheu ${ }^{1}$

${ }^{1}$ Thomas Jefferson University, Philadelphia, Pennsylvania, USA; ${ }^{2}$ University of Glasgow, Glasgow, United Kingdom; ${ }^{3}$ University of Rochester, Rochester, New York; ${ }^{4}$ University of Washington, Seattle, Washington, USA; ${ }^{5}$ University of Padua, Padua, Italy

Mitochondrial $\mathrm{Ca}^{2+}\left(\right.$ mitoCa $\left.{ }^{2+}\right)$ controls the balance of cell survival and death. The molecular identity of the mitoCa ${ }^{2+}$ uniporter (MCU) has been just reported and basal tyrosine phosphorylation of human MCU was shown in mass spectroscopy. However, the functional relevance of MCU phosphorylation is still unknown. Here we showed that 1 -adrenoceptor (1-AR) signaling induces mitoCa ${ }^{2+}$ overload, mitochondrial superoxide $\left(\mathrm{mitoO}_{2}^{-}\right)$ generation and cell death signaling activation through MCU tyrosine phosphorylation in cardiomyocytes. 1-AR signaling activates mitochondrialocalized proline-rich tyrosine kinase $2(\mathrm{Pyk} 2)$ and accelerates mitoCa ${ }^{2+}$ uptake via Pyk2-dependent MCU phosphorylation. Moreover, mitoCa ${ }^{2+}$ overload via this signaling stimulates mitoO2- generation, permeability transition pore activity, and initiates apoptosis. Thus, 1-AR-Pyk2-MCU signaling may become novel potent therapeutic targets for preventing mitoCa ${ }^{2+}$ overload, oxidative stress and cardiac injury under persistent AR stimulation such as during heart failure.

Acknowledgements: AHA grant

\section{5}

\section{SEX-BASED DIFFERENCES IN CARDIAC TOLERANCE TO ISCHEMIC INJURY}

B Ostadal

Institute of Physiology, Academy of Sciences of the Czech Republic, Prague, Czech Republic

Many epidemiological studies have demonstrated that premenopausal women have a reduced risk for ischemic heart disease (IHD) compared with 
their male counterparts. The incidence in women increases after menopause, suggesting that IHD is related to declining estrogen levels. Experimental observations have confirmed the results of epidemiological studies investigating sex-specific differences in cardiac tolerance to ischemia. Detailed mechanisms of these differences remain unknown; however, they involve the genomic and non-genomic effects of sex steroid hormones, particularly the estrogens, which have been studied the most extensively. Although the protective effects of estrogen have many potential therapeutic implications, clinical trials have shown that estrogen replacement in postmenopausal women may actually increase the incidence of IHD. The results of these trials have illustrated the complexity underlying the mechanisms involved in sex-related differences in cardiac tolerance to ischemia. Although our understanding of this important topic has increased in recent years, there is an urgent need for intensive experimental and clinical research to develop female-specific therapeutic strategies. Only then we will be able to offer future patients better evidence-based treatment, a better quality of life and lower mortality.

\section{6}

\section{RACE, SOCIOECONOMIC STATUS, AND RISK OF SUDDEN CARDIAC DEATH}

K Reinier ${ }^{1}$, C Teodorescu' ${ }^{1}$, A Uy-Evanado' ${ }^{1}$, E Stecker ${ }^{2}$,

A Huertas-Vazquez ${ }^{1}$, R Mariani ${ }^{1}$, K Gunson ${ }^{2}$, J Jui ${ }^{2}$, SS Chugh ${ }^{1}$, ${ }^{1}$ Cedars-Sinai Heart Institute, Los Angeles, California; ${ }^{2}$ Oregon Health and Science University, Portland, Oregon, USA

Sudden cardiac death (SCD) is a major contributor to mortality in North America. Data indicates that the burden of SCD may be greater among blacks than among other groups, and greater among people of lower socioeconomic status (SES). Several studies, including recent data from our research group (the Oregon Sudden Unexpected Death Study) have shown that SCD rates among blacks are roughly double the rates among whites. Similarly, rates of SCD are nearly double in neighborhoods in the lowest quartile of median household income compared to the highest. While there is evidence of a higher cardiovascular risk factor burden among blacks and people of lower SES, differences in risk factors may not explain all of the observed differences in rates. An improved understanding of reasons for differences in risk (including potential gene-environment interactions) may identify opportunities for prevention. In addition, neighborhood SES may be an important consideration for effective deployment of community-based prevention strategies as well as placement of automated external defibrillators.

Acknowledgments: Funded by NHLBI.

\section{7}

\section{INVESTIGATING THE REGENERATIVE POTENTIAL OF THE MRL MOUSE STRAIN'S MITOCHONDRIAL GENOME} N Roberts ${ }^{1}$, T Berhanu ${ }^{1}$, A Mull ${ }^{1}$, J Holey-Cuthrell ${ }^{1}$, E McNally ${ }^{2}$, A Heydemann ${ }^{1}$

${ }^{1}$ Department of Physiology and Biophysics and the Center for Cardiovascular Research, University of Illinois at Chicago; ${ }^{2}$ Department of Medicine, University of Chicago, Chicago, USA

We have been pursuing the remarkable ability of the MRL mouse strain to resist muscular dystrophy-mediated fibrosis. For a number of scientific reasons, we have focused upon the genetic polymorphisms found in the MRL mitochondria and their effects upon the metabolic and regenerative characteristics of this mouse strain. Initially we identified that maternal inheritance from the MRL line correlated with reduced muscular dystrophy-mediated fibrosis. We have also identified a number of metabolic differences in the wildtype MRL mice including increased pAMPK, mitochondria, glycolysis, and decreased reactive oxygen species. We have generated 4 backcrossed breeding lines which separate the MRL nuclear and mitochondrial genomes using the very fibrotic DBA2/J as the opposing strain (nuc ${ }^{\mathrm{MRL}}$ mito $^{\mathrm{M}}$, nuc ${ }^{\mathrm{M}}$ mito $^{\mathrm{DBA}} / \mathrm{J}$, nuc ${ }^{\mathrm{D}}$ mito $^{\mathrm{M}}$ and nuc $^{\mathrm{D}} \mathrm{mito}^{\mathrm{D}}$ ). We are now poised to investigate the regenerative molecular signaling cascade initiated by the MRL mitochondrial genome. In the sixth generation of the four backcrossed lines we are analyzing ear wound healing, muscular dystrophy-mediated fibrosis, and metabolic characterizations.

\section{8}

\section{ROLE OF LIPOPROTEIN LIPASE (LPL) IN FATTY ACID (FA) DELIVERY TO THE DIABETIC HEART}

\section{B Rodrigues}

Faculty of Pharmaceutical Sciences, University of British

Columbia, Vancouver, British Columbia

Pharmaceutical management of diabetes can never completely duplicate the exquisite control of glucose observed in healthy humans and hence, patients with diabetes who have imperfect control of their glucose are exposed to repeated bouts of hyperglycemia. As a consequence, changes in cardiac metabolism (decreased glucose utilization forces the heart to increase LPL at the vascular lumen to augment FA) arise, are predicted to occur rapidly, and can influence the development of cardiovascular disease if sustained. The amplification in LPL emerges as an outcome of enzyme movement to the cardiomyocyte cell surface, with subsequent forward transfer to the coronary lumen. We suggest that this occurs due to high glucose induced secretion of endothelial heparanase that has concurrent functions. These include stimulation of myocyte HSPG bound LPL, and VEGF release. The latter effect contributes towards augmenting LPL action, and amplifying FA delivery and utilization by the diabetic heart. At the apical side of EC, LPL is responsible for VLDL hydrolysis to FA, which we propose can result in lipotoxicity. By gaining further insight into the mechanism(s) by which diabetes alters endothelium-bound LPL, we can attempt to piece together a part of the cascade of events leading to diabetic heart disease.

\section{9}

SERIOUS NEW CONCERNS FOR PATIENTS WITH PERIPHERAL ARTERIAL DISEASE

D Rodriguez-Leyva ${ }^{1}$, ${ }^{2}$ Guzman ${ }^{2}$, A Rodriguez-Portelles ${ }^{1}$, GN Pierce ${ }^{2}$

${ }^{1}$ Cardiovascular Research Division, Holguin University Hospital, Cuba; ${ }^{2}$ St Boniface Hospital Research Centre, Winnipeg, Manitoba A susceptibility to arrhythmias may explain the increased risk for cardiovascular events in patients with peripheral artery disease (PAD). The purpose of the study was to identify the incidence and type of arrhythmias in PAD patients and their relationship with vascular abnormalities. In an observational cross sectional study we examined 110 PAD patients $(73 \%$ male, 67 years old) with an ankle/brachial index $(\mathrm{ABI})$ of $<0.9$. EKG evidence of arrhythmias was identified at rest, during exercise and post-exercise. Augmentation index (AI) values were also measured by pulse wave analysis. Cardiac arrhythmias were found in $37 \%$ of the subjects. Frequent premature ventricular and supraventricular contractions were the most common arrhythmias (78\%). Most (65\%) were observed at rest. PR segment, QRS complex and QTc interval were significantly longer in patients with arrhythmias. QTc differences were significant for males but not for females. Age ( $>60$ years), pre-existing coronary artery disease (CAD) and greater arterial stiffness were identified as predictors of arrhythmias. Patients with PAD have a high incidence of cardiac arrhythmias. Older age, concomitant $\mathrm{CAD}$ and greater arterial stiffness may pre-dispose PAD patients to arrhythmias.

\section{0}

SYSTEMATIC REVIEW OF NUTRITION AND CARDIOVASCULAR DISEASES

\section{A Rodriguez-Portelles, D Rodriguez-Leyva}

Division of Cardiology, Holguin University of Medicine, Cuba

Cardiovascular diseases are considered a global pandemic. Poor nutrition favors the appearance of these as a proper diet has a positive effect on its prevention and control. It is critical to translate current nutrition knowledge into effective strategies to prevent and treat these diseases. This systematic review was conducted in order to identify nutritional factors associated with cardiovascular diseases and susceptible to intervention at the family physician level of care. Thirty trials referenced by PubMed and Cochrane in the last 5 years were reviewed using the key words "nutrition", "cardiovascular risk factors" and "cardiovascular prevention". A second experiment consisted in a three months nutritional educative intervention at a family physician office based on the results of the systematic review. There was consensus that the 
ideal diet for primary and secondary prevention in patients with cardiovascular disease should be low in saturated fat, cholesterol and trans fatty acids; rich in fruits, vegetables, fatty fish, chicken, lean meat, legumes, cereals and whole grains. At the primary level of care family physicians can positively impacts the course of cardiovascular diseases by a timely nutritional intervention. Acknowledgements: St. Boniface Hospital Research Centre

\section{1}

\section{NOVEL BIOACTIVE OXIDIZED PHOSPHOLIPIDS ARE PRODUCED IN MYOCARDIUM DURING ISCHEMIA REPERFUSION AND ACT AS MEDIATORS OF CELL DEATH WITHIN CARDIAC MYOCYTES}

D Hasanally, D Chan, V Margulets, R Chaudhary, S Premecz, DS Jassal, LA Kirshenbaum, A Ravandi

St Boniface Research Centre, Winnipeg, Manitoba

A major therapeutic aim for myocardial infarction is early 'reperfusion' of ischemic tissue. The process of ischemia followed by reperfusion (IR) causes an intense oxidative burst. the impact of increased oxidative stress on cardiac celluar lipids is unkown. In rat model of IR injury lipidomics analysis revealed a significant increase in total oxidized phosphatidylcholine (OxPCs) after $1 \mathrm{~h}$ of IR with $24 \mathrm{~h}$ of IR resulting in a significant sustained increase of fragmented OxPCs $(1.80 \pm 0.22$ fold, $\mathrm{P}<0.05)$. Cardiomyocytes under $\mathrm{I} / \mathrm{R}$ conditions also demonstrated significant increase in total OxPCs $(5.23 \pm 0.99$ fold vs control, $\mathrm{P}<0.05)$ and in fragmented aldehyde species $(1.52 \pm 0.11$ fold, $\mathrm{P}=0.05)$ correlating with decreased cell viability. Exogenously added fragmented OxPC such as POVPC resulted in a significant loss of cardiomyocyte viability after $4 \mathrm{~h}$ of exposure when compared to control non oxidized PC, PSPC $(46 \pm 5 \%$; $40 \pm 5 \%$, respectively, vs $9 \pm 3 \% \mathrm{PSPC}, \mathrm{P}<0.05)$. Exposure of cardiomyocytes to POVPC and PONPC resulted in increased mitochondrial permeability and DNA fragmentation. We have shown for the first time that OxPCs are generated within myocardial tissue during IR and they have detrimental effects on cardiomyocytes.

\section{2}

\section{LIFESTYLE-RELATED CARDIOVASCULAR DISREGULATIONS ARE ASSOCIATED WITH LOSS OF INNATE ANTIISCHEMIC PROTECTION}

T Ravingerova ${ }^{1}$, V Ledvenyiova ${ }^{1}$, M Murarikova ${ }^{1}$, S Griecsova ${ }^{1}$,

S Carnicka', M Zalesak', A Adameova ${ }^{2}$

${ }^{1}$ Institute for Heart Research, Slovak Academy of Science \& Centre of Excellence, SAS NOREG; ${ }^{2}$ Faculty of Pharmacy, Comenius University, Bratislava, Slovak Republic

Lower tolerance to ischemia/reperfusion injury (IR) was shown in subjects with lifestyle risk factors (RF), eg, metabolic disorders or aging. However, stimuli like ischemia, hyperglycemia or free radicals trigger preconditioning (PC) rendering the heart more resistant to IR. It is hypothesized that RF may modify response to ischemia not only by interfering with mechanisms of IR per se, but also suppress PC promoting pro-oxidative and apoptotic processes that shift balance from cell survival to cell death. Protection is blunted in the preconditioned or acutely diabetic hearts with comorbidities and in elder animals. Loss of innate cardioprotection is associated with decline in PC-induced upregulation of pro-survival PI3K/Akt, eNOS, PKC $\varepsilon$ proteins and PPAR genes. Nevertheless, potential of PC is still retained in the pathologically altered myocardium. Thus, a higher intensity of an adaptive stimulus may be required to reach a similar level of protection as in the 'healthy' heart, by employing a higher number of PC cycles/concentration of substances that mimic PC, by novel and safer modifications of PC including 'remote' or 'delayed' PC or by their combinations.

Funding: Grant APVV-0102-1.

\section{3}

\section{CARDIOVASCULAR GENETICS - A GLIMPSE OF MEDICINE IN THE FUTURE}

\section{R Roberts}

University of Ottawa Heart Institute, Ottawa, Ontario

Fifty genetic risk variants were found to be associated with coronary artery disease $(\mathrm{CAD})$ of genome-wide significance in the discovery population and replicated in an independent population. Each of these risk variants are extremely common with more than half occurring in $>50 \%$ of the general population. They increased only minimally the relative risk for CAD. The most striking finding is that 35 of the 50 risk variants act independently of known risk factors, indicating there are several pathways yet to be appreciated, contributing to the pathogenesis of coronary atherosclerosis and myocardial infarction. All appear to act through atherosclerosis except for the $\mathrm{ABO}$ blood type with $\mathrm{A}$ and $\mathrm{B}$ increasing the risk for myocardial infarction through increased plasma half life of Von Willebrand factor. The discovery of PCSK9 mutations has led to a new therapy which is currently in Phase III clinical trials. This therapy which increases removal of LDL-C complements statin therapy which decreases synthesis. Mendelian randomization provides a new approach to assess safety and efficacy of potential novel therapies.

\section{4}

\section{SCLERAXIS MEDIATES THE CARDIAC MYOFIBROBLAST PHENOTYPE}

P Roche, R Bagchi, N Aroutiounova, M Czubryt

Department of Physiology \& Pathophysiology, University of Manitoba, Winnipeg, Manitoba

Myofibroblasts are the primary mediators of cardiac remodeling and fibrosis, for which there is currently no treatment. Scleraxis is a transcription factor regulating development of collagen-rich tissues. In the heart, scleraxis expression is increased by the same stimuli that induce myofibroblast differentiation, in vitro and in vivo. Thus, we hypothesize scleraxis mediates the myofibroblast phenotype. Using adenovirus, we over-expressed and knocked down scleraxis in primary cardiac fibroblasts $(\mathrm{CFs})$. Myofibroblast marker expression increased with scleraxis over-expression, and decreased with knockdown. We then examined functional myofibroblast characteristics (contractility, reduced migration) in response to changes in scleraxis expression. Using a gel contraction assay, we determined that scleraxis is capable of inducing CF contraction and is also required for CF contraction induced by TGF- $\beta$ (a strong inducer of the myofibroblast). Additionally, transwell migration experiments showed that scleraxis represses CF migration, and this inhibition is relieved by scleraxis knockdown. These results strongly implicate scleraxis as a regulator of the myofibroblast, and a novel target for pharmacological inhibition.

Funding: Provided by CIHR.

\section{5}

\section{VAGUS NERVE STIMULATION FOR TREATMENT OF HEART FAILURE}

\section{HN Sabbah}

Department of Medicine, Henry Ford Hospital, Detroit, Michigan, USA Autonomic dysregulation is an important feature of chronic heart failure (HF) and is characterized by a sustained increase of sympathetic drive and by withdrawal of parasympathetic activity. Increased circulating levels of norepinephrine and increased heart rate (HR) are both predictors of poor longterm outcome in patients with HF. Pharmacologic agents that partially inhibit sympathetic overdrive such as beta-adrenergic receptor blockers are effective in reducing mortality and morbidity in patients with chronic HF. More recently, modulators of parasympathetic activity have gained more attention as potential therapeutics for HF. Studies in animals with experimental HF have shown that chronic electrical stimulation of the Vagus nerves (VNS) improves LV systolic and diastolic function and reverses LV chamber remodeling. In addition to lowering sympathetic drive and reducing HR in HF, VNS was also shown to 1) suppress excessive elaboration of proinflammatory cytokines; 2) normalize nitric oxide signaling; and 3) reduce the threshold for development of life threatening ventricular arrhythmias Multiple clinical trials are currently underway to evaluate the safety and efficacy of VNS in patients with chronic HF.

Acknowledgements: Grant funding provided by NHLBI and BioControl Medical, Inc. 


\section{6}

\section{CARDIOVASCULAR TOXICITY OF CARFILZOMIB ON VASCULAR TONE, VASCULAR REACTIVITY AND ENDOTHELIAL FUNCTION}

TM Scarabelli ${ }^{1}$, M Gavazzoni ${ }^{2}$, C Chen-Scarabelli ${ }^{3}$, G Sahni ${ }^{1}$, J Narula ${ }^{1}$, R Raddino ${ }^{2}$

${ }^{1}$ The Zena and Michael A Wiener Cardiovascular Institute, Icahn School of Medicine at Mount Sinai, New York, USA; ${ }^{2}$ Division of Cardiology, University of Brescia, Italy; ${ }^{3}$ VA Ann Arbor Health Care System, University of Michigan, Ann Arbor, Michigan, USA

Carfilzomib (CFZ) is a new proteasome inhibitor used for the treatment of Multiple Myeloma. Cardiac failure events and myocardial ischemia have occurred following administration CFZ. Infusion reactions also include chest tightness and angina of unknown mechanism. Here, we investigated whether CFZ exerts in vitro effects on vascular tone and reactivity in an isolated experimental model of rabbit thoracic aortic-strips. CFZ increased the resting vasoconstricting tone and amplified the spasmogenic effect of different agents, including potassium chloride, noradrenaline and angiontensin II. Moreover, preincubation with CFZ decreased the anti-spasmogenic activity of nitroglycerine and reduced by over $50 \%$ the vasodilating effect of acytocholine, suggesting that CFZ can impair vasodilation by inducing endothelial dysfunction. In conclusion, further studies are warranted to establish its clinical safety in patients with known CAD and prior history of coronary spasm.

\section{7}

\section{MECHANISM UNDERLYING TAURINE DEFICIENT CARDIOMYOPATHY}

\section{SW Schaffer, KC Ramila}

\section{University of South Alabama, Mobile, Alabama, USA}

Taurine is a beta-amino acid found in very high concentration in the heart. A key function of taurine its conjugation with a wobble position uridine of mitochondrial tRNA(leu), a reaction that affects the strength of the interaction between the codon and anticodon of tRNA(leu). Hence, taurine deficiency alters mitochondrial protein synthesis and respiratory chain function. Taurine deficient animals containing reduced mitochondrial taurine levels develop a cardiomyopathy characterized by diminished contractile function, reduced ventricular wall thickness and cardiomyocyte atrophy. However, the mechanism responsible for the development of this unique combination of changes remains unknown. We found that both sarcoplasmic reticular Ca ATPase activity and the phosphorylation state of phospholamban were reduced in taurine deficient hearts. These changes were associated with reduced CaMKII (calmodulin-dependent protein kinase II) activity and an elevation in protein phosphatase I activity. Although taurine deficiency leads to oxidative stress, SERCA2a levels were normal in the taurine deficient heart. Severe taurine deficiency also leads to impaired mitochondrial function, which diminishes ATP production, increases the degree of oxidative stress and promotes apoptosis, factors that also appear to contribute to the development of the cardiomyopathy.

\section{8}

\section{ROLE OF OMEGA-3 FATTY ACIDS FOR THE PREVENTION} AND MANAGEMENT OF CARDIOVASCULAR DISEASE

\section{NA Shaikh ${ }^{1}$, G Jackowski ${ }^{2}$}

1University of Toronto (Ret), Toronto; ${ }^{2}$ Pivotal Therapeutics Inc, Woodbridge, Ontario

BACKGROUND: Low blood levels of Omega-3 fatty acids $(\omega-3)$ have been associated with increased risk for cardiovascular disease (CVD). However, recent meta-analyses of studies have raised some doubts on the efficacy of $\omega-3$ for management of CVD, most likely due to variable doses, ratio and purity of $\omega-3$, to patient selection and drug milieu and lack of monitoring of pre- or post treatment $\omega-3$ levels. The present talk summarizes the relevant studies and further proposes that patient response to $\omega-3$ is also an important factor.

METHOD \& RESULTS: A randomized controlled trial consisting of 110 enrolled subjects. Primary Endpoint: Correction of $\omega-3$ deficiency as blood $\omega-3$ levels (Omega-Score, $\mathrm{OS}=\mathrm{EPA}+\mathrm{DHA}+\mathrm{DPA}$; eicosapentaen oic +docosahexaenoic+docosapentaenoic acids) measured at 0 and after 8 weeks of treatment with $4 \mathrm{~g} /$ day of a $90 \%$ pure $6: 1 \mathrm{EPA} / \mathrm{DHA}$ formulation or with corn oil (placebo). Secondary Endpoint: change in blood lipid profiles. In both normal and high-TG subjects, median \% increase in OS was comparable (132 vs 128) in both groups but the response greatly varied among responders (50\% to $195 \%$ ).

CONCLUSION: The purity, EPA:DHA ratio, dose and patient's response are the determinant factors for optimal efficacy of a formulation in reducing CVD risk factors.

\section{9}

\section{ADRENERGIC CONTROL OF CARDIAC FATTY ACID OXIDATION IN DIABETES}

\section{Sharma}

Department of Pathology, Aberdeen Royal Infirmary and University of Aberdeen, Aberdeen, United Kingdom

Diabetes produces a direct and continuous myocardial insult even in the absence of ischemic, hypertensive or valvular disease. Beta-blockers have been shown in large-scale randomized controlled trials to reduce heart failure mortality. In the diabetic heart, metoprolol inhibits fatty acid oxidation but does not prevent lipotoxicity; its beneficial effects are more likely to be due to anti-apoptotic effects of chronic treatment. Two intriguing mechanisms of these effects are emerging. The first is USF-2-mediated repression of PGC- $1 \alpha$. In addition, preliminary data hint at a possible role for caveolins. The range of effects produced by beta-adrenergic blockade are broad and illustrate how interconnected the signalling pathways of function and metabolism are in the heart.

Acknowledgements: This work was funded by the Canadian Institutes of Health Research and the Heart and Stroke Foundation of Canada.

\section{0}

\section{BREAKING DOWN CELL CONNECTIONS TO UNRAVEL NEW INSIGHTS IN ARRHYTHMOGENIC HEART DISEASE F Sheikh}

Department of Medicine, University of California-San Diego, La Jolla, California, USA

Arrhythmogenic right ventricular cardiomyopathy (ARVC) termed a "disease of the desmosome" recently underwent reclassification due to the identification of left dominant and biventricular disease forms. Homozygous loss-of-function mutations in the desmosomal component, desmoplakin (DSP), are found in patients exhibiting a biventricular form of ARVC; however no models recapitulate the disease as seen in these patients. To determine the homozygous loss-of-function effects of DSP in the heart, we generated a novel cardiac-specific DSP-deficient mouse (DSP-cKO). DSPcKO mice were viable but display early desmosomal defects leading to a biventricular form of ARVC that includes fibrofatty replacement of the ventricle, biventricular dysfunction, failure and death. DSP-cKO mice exhibited conduction abnormalities such as (i) ventricular arrhythmias that are exacerbated with exercise and catecholamines, (ii) right ventricular conduction defects associated with loss of connexin 40 and (iii) electrical wavefront propagation defects associated with loss of connexin 43. In vitro studies in DSP deficient cardiomyocytes revealed that defects in connexin 43 precede the molecular breakdown of the cell junction complex and tissue remodeling, highlighting early mechanisms underlying ARVC. Supported by NIH and AHA.

\section{1}

\section{EFFECT OF PRE, MODERATE AND CHRONIC DIABETES MELLITUS ON FIBROSIS AND HYPERTROPHY IN THE RAT HEART}

J Singh ${ }^{1}$, A D'Souza ${ }^{1}$, K Bidasee ${ }^{2}$, C Howarth ${ }^{3}$, E Adeghate ${ }^{4}$, T Iqbal $^{1}$

'Schools of Pharmacy, Forensic and Investigative Sciences, UCLAN, Preston, United Kingdom; ${ }^{2}$ Pharmacology and Neurosciences, University of Nebraska Medical Centre, Omaha, Nebraska, USA; ${ }^{3}$ Physiology; ${ }^{4}$ Anatomy, United Arab Emirates University, Al Ain, United Arab Emirates

Diabetes-induced chronic hyperglycaemia (HG) causes adverse cardiac structural remodelling. This study compares the left ventricle (LV) of 2-month (pre-diabetes) and 18-month (moderate) old Goto-Kakizaki 
(GK) rats and 2 and 4 months STZ-induced diabetic (chronic) rats with controls to investigate the hypothesis that transforming growth factor beta 1 (TGF-beta1), natriuretic peptides (ANP, BNP), pro-hypertrophic markers Akt-mTOR-p70S6K1 and extracellular matrix (ECM) factors are linked to fibrosis. GK rats had significantly $(\mathrm{P}<0.05)$ increased heart weights, heart weight to body weight ratio, LV free wall thickness and myocyte diameter compared to their respective controls, an indication of marked cardiac hypertrophy. These parameters were significantly $(\mathrm{P}<0.05)$ less in STZ-induced diabetic rats. Significant $(\mathrm{P}<0.05)$ increases in TGFbeta1, BNP, ANP, Akt-mTOR: p70S6K and gene expressions for ECM factors was observed. Thus, pre-diabetes, moderate and chronic HG can evoke severe adverse remodelling of the LV indicating detrimental effects of $H G$ to cardiac structure and function.

\section{2}

\section{TREATMENT WITH BMP-7 ENHANCES M2 MACROPHAGES AND REDUCES PRO-INFLAMMATORY CYTOKINES AND PLAQUE FORMATION IN APOE KNOCKOUT MICE \\ D Singla \\ Burnett School of Biomedical Sciences, Orlando, Florida, USA}

We have determined the effects of BMP-7 on monocytes differentiation and M1 vs M2 macrophage phenotype polarization in ATH. ApoE KO mice were purchased, and used to partial left corotid artery ligation (PLCA) to develop ATH. Male and female mice were divided into three groups: Sham control, PLCA, and PLCA+BMP-7. Animals were given BMP-7 or saline intravenously (i.v.). Echocardiography was recorded, and ELISA's were performed. Treated animals with BMP-7 significantly $(\mathrm{P}<0.05)$ reduced plaque formation. Echocardiography data shows significantly $(\mathrm{P}<0.05)$ reduced blood velocity in ATH group compared with controls. However, following treatment with BMP-7 the blood velocity was improved. Immunostaining data suggest that a significant increase in monocytes in the PLCA group compared to controls, and reduction in monocyte was evident in PLCA+BMP-7 group. Next, our data on iNOS specific for M1 macrophages, and CD206 for M2 macrophages shows significantly $(\mathrm{P}<0.05)$ increased M2 macrophages in BMP-7 treated animals. Furthermore, ELISA data suggest decrease in proinflammatory cytokines and increase in anti-inflammatory cytokines. In conclusions, we suggest that animals treated with BMP-7 attenuate plaque formation, improved blood velocity in ATH.

\section{3}

\section{CARDIOVASCULAR RADIATION INJURY AND POTENTIAL TARGETS FOR PREVENTION}

\section{LSlezak ${ }^{1,2}$}

\section{Institute for Heart Research, SAS Bratislava; ${ }^{2}$ Slovak Medical} University, Bratislava, Slovak Republic

Exposure of oncological patients to therapeutic doses of chest irradiation has been significant source of cardiovascular morbidity and mortality. The cellular response to radiation injury initiates an active process that ultimately leads to progressive damage. This process can be modulated by therapies directed at mitigating the cascade of events resulting from normal tissue injury. The aim of the study was to explore the effects of a single dosage radiation ( 25 Gy) applied to the mediastinal area of normal adult Wistar rats on the heart functional parameters and selected molecular markers 6 weeks after irradiation. Irradiation of normal tissues leads to increases in reactive oxygen and reactive nitrogen oxide species that serve as intracellular signaling to alter cell function, resulting in chronic inflammation, progressive damage, organ dysfunction, and ultimate failing of the heart. It can be concluded that 6 weeks after irradiation, despite progressive deterioration of the animals and endothelial cell damage, adaptive and/or compensatory mechanisms are still working and cardiac function is maintained. On the basis of our results, selected substances were tested. Funding: Supported by grant: APVV-0241-11
114

\section{ROLE OF CALMODULIN-DEPENDENT PROTEIN KINASE II IN ET-1-INDUCED RESPONSES IN VSMC}

\section{AK Srivastava}

Research Centre, CHUM, Montreal, Quebec

A multifunctional calcium-calmodulin-dependent protein kinase II (CaMKII) has emerged as an important transducer of vasoactive peptideinduced responses in vascular smooth muscle cells (VSMC). ET-1, a potent vasoactive peptide with a pathogenic role in vascular diseases, has been shown to induce the activation of ERK1/2, PKB and the expression of a transcriptional regulator, the early growth response 1 (Egr-1), key mediators of hypertrophic and proliferative responses in VSMC.However, an involvement of CaMKII in mediating these responses in VSMC remains unknown. Therefore, by utilizing pharmacological inhibitors and CaMKII knockdown techniques, we have investigated the contribution of CaMKII in ET-1induced ERK1/2 and PKB signaling, Egr-1 expression and hypertrophic and proliferative responses in VSMC. Antagonists of CaM and CaMKII, attenuated ET-1-induced ERK1/2 and PKB phosphorylation. In addition, transfection of VSMC with a CaMKII inhibitory peptide or siRNA-mediated CaMKII silencing suppressed ET-1-evoked ERK1/2 and PKB phosphorylation, protein and DNA synthesis as well as Egr-1 expression in VSMC. These data demonstrate that CaMKII, by regulating ET-1-induced signaling pathways and hypertrophic as well as proliferative responses, plays a key role in vascular pathophysiology.

Funding: Supported by CIHR.

115

\section{THE RELATIONSHIP BETWEEN PRE-ARREST COMORBIDITIES AND SURVIVAL AFTER SUDDEN CARDIAC ARREST}

EC Stecker ${ }^{1}$, C Teodorescu ${ }^{2}$, K Reinier ${ }^{2}$, A Uy-Evanado ${ }^{2}$, R Mariani ${ }^{2}$, H Chugh ${ }^{2}$, K Gunson ${ }^{1}$, J Jui ${ }^{1}$, SS Chugh ${ }^{2}$ ${ }^{1}$ Oregon Health and Science University, Portland, Oregon; ${ }^{2}$ Cedars-Sinai Heart Institute, Los Angeles, California, USA

While several resuscitation-related factors are consistently associated with survival from sudden cardiac arrest (SCA), the impact of specific comorbid conditions has not been assessed. As part of the Oregon Sudden Unexpected Death Study (SUDS), we examined the relationship between pre-arrest comoribities and survival after SCA. Among 1466 included patients, CAD predicted higher odds of survival (unadjusted OR 1.5, $\mathrm{P}<0.001$; adjusted $\mathrm{OR} 1.5, \mathrm{P}=0.02$ ), while none of the other comorbidities were associated. These findings raise novel mechanistic questions regarding survival after SCA.

\section{6} METABOLIC RATE DEPRESSION: THE HEART IN WINTER KB Storey

Department of Chemistry, Carleton University, Ottawa, Ontario

Strong metabolic rate depression, often to just 1-5\% of normal resting rate, forms the core of mammalian hibernation. Studies by my lab have shown that conserved mechanisms support prolonged torpor by heart: (a) global suppression of enzymes by reversible protein phosphorylation, (b) reprioritization of ATP use to sustain crucial cell functions while allowing others to lapse, (c) increased expression of selected genes, and (d) enhanced cell preservation mechanisms (e.g. chaperones, antioxidants) to deal with abiotic stress during torpor. New work is showing that epigenetic mechanisms also supply reversible suppression of gene expression during torpor. Changes in global DNA methylation patterns, posttranscriptional modifications of histones (acetylation, methylation, phosphorylation), and altered enzyme activities (e.g. HDACs, DNMTs) also occur during torpor to suppress gene transcription. Sirtuin deacetylases that affect many metabolic enzymes also contribute to hypometabolism, adding evidence that reversible protein acetylation is as important to metabolic control as phosphorylation. Posttranscriptional controls on mRNA are also crucial including inhibition by microRNAs and transcript storage into subnuclear particles. More information at www.carleton.ca/ kbstorey. Acknowledgements: Grant funding by NSERC and the Heart \& Stroke Foundation of Canada. 


\section{7}

\section{HDACS AND BETS CROSSTALK TO REGULATE CARDIAC HYPERTROPHY}

M Stratton, J Spilotoir, K Demos-Davies, B Ferguson, M Cavasin, TA McKisney

Division of Cardiology, UC Denver, Denver, Colorado, USA

The BET family of proteins recognize and bind acetylated lysine residues on histones via conserved bromodomains. One of these BET proteins, BRD4 recruits components of pTEF $\beta$ to promoters of activated genes. pTEF $\beta$ subsequently phosphorylates RNA Pol II, causing release from its stalled state and leading to mRNA production. We have shown that inhibiting BET protein and acetyl lysine interactions with the small molecule JQ1 blocked cardiac remodeling in a TAC pressure overload mouse model. In addition, we report that BRD4 protein expression is upregulated under conditions of pathologic hypertrophy including human heart failure. Despite increased protein expression we found no change in BRD4 mRNA, suggesting post transcriptional regulation. We have also shown that BRD4 binds to the promoter of a prototypical fetal cardiac gene, ANF, in response to hypertrophic stimulus. Inhibition of enzymes that remove acetyl marks from lysine residues on histones (HDACs) abolished this recruitment. Moreover, treatment with the class I specific HDAC inhibitor, MGCD0103, blocked PE induced increases in BRD4 protein expression without altering mRNA expression. We propose a model in which HDACs regulate BRD4 dependent gene expression via targeting of BRD4 to promoters of active genes and regulation of BRD4 protein expression.

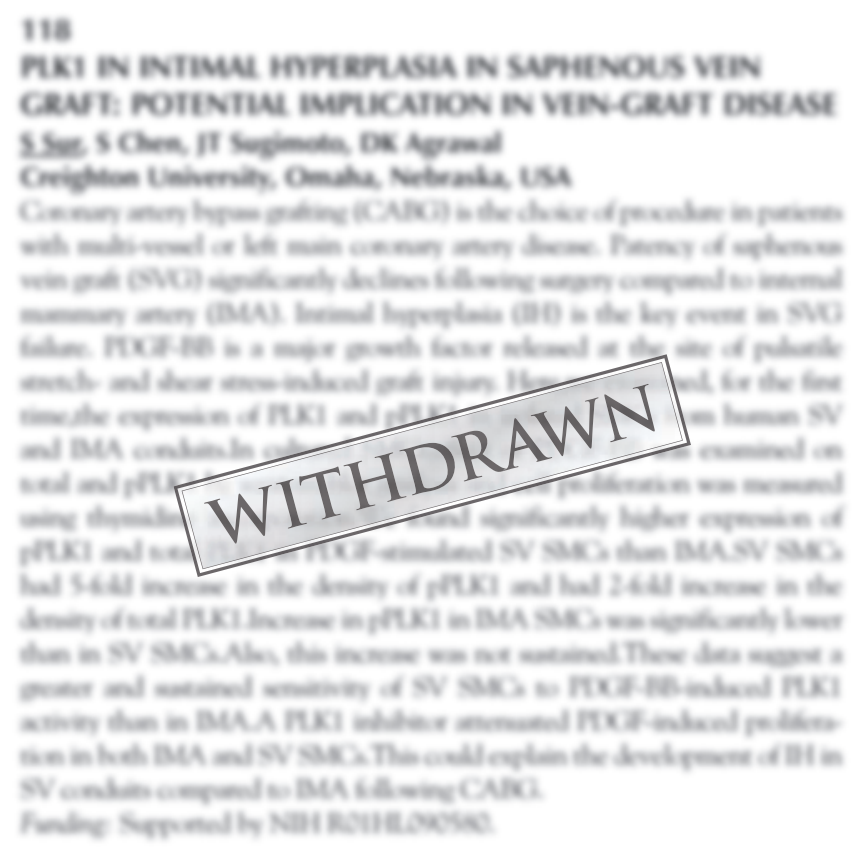

\section{9}

\section{HAND HELD ECHOCARDIOGRAPHY: FAD, FACT OR FICTION?}

\section{JW Tam}

St Boniface General Hospital, University of Manitoba, Winnipeg, Manitoba

Echocardiography is a standard, widely proven, noninvasive technique in the assessment of patients with known or suspected cardiac disease. Rapid evolution of miniature devices with improved image quality has allowed for widespread dissemination of hand held echocardiography for focused cardiac ultrasound assessment in a wide variety of patient encounters. Accuracy in the hands of trained experts parallels that of the use of full service, larger units, and is highly superior to standard physical examination. The optimal degree, time and intensity of training of novice users remains undetermined. The literature demonstrates mixed results, dependent on patient selection, clinical setting, and variable competence of the users. Ease of use, competitive pricing and enhanced imaging options, however, is sure to make this technology a keeper for the future. A suggested role for optimal integration of this technology within the current and future health care system will be presented.

\section{ROLE OF ESTROGEN REPLACEMENT THERAPY IN THE DEVELOPMENT OF HEART FAILURE}

\section{PS Tappia ${ }^{1,2}$, NS Dhalla1,3}

${ }^{1}$ St Boniface Hospital Research Centre; ${ }^{2}$ Asper Clinical Research Institute; ${ }^{3}$ Institute of Cardiovascular Sciences, University of Manitoba, Winnipeg, Manitoba

Although gender differences for several etiologies of heart failure are well documented, there is no in vivo study identifying sex differences in failing hearts due to volume overload. Ovariectomized (OVX) female rats exhibited altered hemodynamic and cardiac remodeling similar to male animals at 16 weeks post-AV shunt, which were attenuated by $17-\beta$ estradiol. A significant increase in apoptosis 16 weeks post-AV shunt was seen in the male rats whereas it was lower in the females. The decrease in apoptosis was abolished by ovariectomy; however, apoptotic levels decreased to those similar to intact female with 17- $\beta$ estradiol. Alterations in components of the $\beta$-adrenergic signaling system were also observed. While both $\beta_{1}$ - and $\beta_{2}$-adrenoceptor protein content and mRNA levels as well as other elements of the $\beta$-adrenergic signaling system were decreased in males, these components were increased in females. However, AV shunt for 16 weeks in OVX female animals resulted in similar changes as seen in males. Treatment of OVX rats with 17- $\beta$ estradiol prevented the AV shunt induced changes in $\beta$-AR and adenylyl cyclase protein content. These results demonstrate the role of estrogen in resistance to heart failure in females.

\section{1}

\section{MECHANISMS OF PULSELESS ELECTRICAL ACTIVITY}

C Teodorescu ${ }^{1}$, K Reinier ${ }^{1}$, A Uy-Evanado ${ }^{1}$, R Mariani ${ }^{1}$, K Gunson ${ }^{2}$, J Jui ${ }^{2}$, S Chugh ${ }^{1}$

${ }^{1}$ Cedars-Sinai Heart Institute, Los Angeles, California; ${ }^{2}$ Oregon Health \& Science University, Portland, Oregon, USA

The manifestation of sudden cardiac arrest (SCA) has changed significantly over time, with a decline in ventricular fibrillation/tachycardia (VF/VT) and an increase in the proportion of SCA cases presenting with pulseless electrical activity (PEA) and asystole. PEA has diverse etiologies listed as the " $5 \mathrm{Hs}$ and 5 Ts". The most common final mechanism is global ischemia caused by a severe reduction in coronary flow. Based on echocardiographic appearance there are two types of PEA: pseudo-PEA characterized by synchronous contractility and true PEA with non-synchronous or non-contractile presentation. Given significantly lower survival from PEA, there is an urgent need for an improved understanding of determinants of PEA to provide opportunities for prevention. In the Oregon Sudden Unexpected Death Study we comprehensively investigated PEA determinants by incorporating first responder data with lifetime clinical history information among SCA cases, and identified significant differences between cases presenting with VF/VT vs. PEA. Our findings indicate that malignant vasodepressor response and prescription drugs may influence the pathophysiology of PEA. Acknowledgements: Grant funding provided by NHLBI.

\section{2}

\section{PROTEOMIC MAPPING OF MMP-9 CLEAVAGE SITES} ON FIBRONECTIN

$\underline{\text { Y Tian }}{ }^{1,2}$, LE de Castro Brás ${ }^{1,2}$, ML Lindsey ${ }^{1,2,3}$

${ }^{1}$ Mississippi Center for Heart Research, Department of Physiology and Biophysics, University of Mississippi Medical Center, Jackson, Mississippi; ${ }^{2}$ San Antonio Cardiovascular Proteomics Center, San Antonio, Texas; ${ }^{3}$ Research Service, GV (Sonny) Montgomery Veterans Affairs Medical Center, Jackson, Mississippi, USA

Matrix metalloproteinase (MMP)-9 deletion protects against dilation of the left ventricle (LV) following myocardial infarction (MI). MMP-9 degrades extracellular matrix (ECM) proteins, and ECM remodeling is a critical wound healing response post-MI. We have previously identified fibronectin, an ECM protein increased in the infarcts post-MI, as an in vivo substrate of MMP-9. Fibronectin is necessary for cell adhesion and migration during LV repair. The goal of the current study was to map MMP-9 cleavage sites on fibronectin using in vitro cleavage assays coupled with a gel-based separation or isotope labeling of neo $\mathrm{N}$-terminal peptides followed by trypsin digestion and mass spectrometry analysis. We identified 21 potential cleavage sites. 
Peptides containing centrally located cleavage sites were synthesized for validation. Eighteen of the 21 cleavage sites (86\%) were confirmed by in vitro analysis. In conclusion, this study reveals the exact MMP-9 cleavage sites on fibronectin for future studies examining the roles of MMP-9 generated fibronectin fragments.

\section{3}

\section{THE SARCOLEMMAL MEMBRANE ASSOCIATED PROTEIN (SLMAP) OVEREXPRESSION LEADS TO IMPAIRED CARDIAC FUNCTION}

M Trentin Sonoda*, F da Silva*, J Major, J Mlynarova, M Salih, MSC Ramos, BS Tuana

Department of Cellular and Molecular Medicine, University of Ottawa, Ottawa, Ontario

The sarcolemmal membrane-associated proteins (SLMAPs) are unique regulators of cardiac excitation-contraction (E-C) coupling. The SLMAP gene is spliced in myocardium and gives rise to many isoforms. Cardiac specific overexpression of the isoform SLMAP-1 was able to interfere with the endogenous SLMAP homodimerization, resulting in vacuolated myocardium and impaired cardiac function. Here we have investigated the role of SLMAP-3 isoform. Transgenic mice with cardiac specific overexpression of SLMAP-3 were generated and examined with echocardiography (echo) to assess functional parameters, qPCR to analyze gene expression and western blots for protein expression. The echo of 6 and 12 weeks old mice did not show any differences between the wild type and transgenic (high SLMAP expression levels) animals. However at 18 weeks of age, a decrease of $\sim 31 \%$ in ejection fraction and $\sim 36 \%$ in fractional shortening was noted. There was no noticeable change in cardiac remodeling at protein or gene expression levels. These data indicate that regulated levels of SLMAP are important for normal cardiac function and that it may potentially be a unique target in cardiac disorders.

Funding: HSFC. *Supported by CAPES.

\section{4}

\section{SEX DIFFERENCES AND DIABETES MELLITUS IN CARDIOVASCULAR FUNCTION}

\section{B Turan}

Department of Biophysics, Ankara University, Faculty of Medicine,

\section{Ankara, Turkey}

Clinical and animals studies reveal importance of sex-differences in cardiac performance/responses under pathology. Although many studies investigated sex-related cardiovascular disorders, most proposed mechanism is focused on distinction in regulation of cellular $\mathrm{Ca}^{2+}$ and oxidative-stress. We studied role of sex-differences in regulation of cardiac $\mathrm{Ca}^{2+}$ homeostasis under physiological and pathophysiological conditions such as hyperglycemia. Under physiological condition and at organ level, there were no significant sex-dependent differences in cardiac function while marked differences were recorded in intracellular $\mathrm{Ca}^{2+}$ regulation at cellular levels. Our animal data showed that one of possible underlying mechanisms of sex-related differences in response to different stimuli in healthy and diabetic subjects were due to distinction in levels of sex-dependent oxidative stress/antioxidant defense ratios. We also demonstrated that cardiac responses to -blockers showed sex-dependent differences under physiological and hyperglycemic conditions. Our data, therefore, pointed out the importance of cellular redox-state in heart function in a manner of both sex- and -age dependent as well as how females' myocardium is more resistant against diabetes-induced cardiac dysfunction compared to males. Acknowledgements: TUBITAK SBAG-113S466 \& -111S042.

\section{5}

TRANS FATTY ACIDS ARE TAKEN UP BY CARDIOMYOCYTES AFTER DIRECT SUPPLEMENTATION TO THE MEDIA

\section{RK La Vallée, R Ganguly, GN Pierce}

Institute of Cardiovascular Sciences, St Boniface Research Centre, Department of Physiology, Faculty of Health Sciences, University of Manitoba, Winnipeg, Manitoba

Industrial trans fats (TF), such as elaidic acid (EA) can be harmful to our health and are linked to atherosclerotic cardiovascular disease. However, naturally produced TF such as vaccenic acid (VA) have beneficial effects on atherosclerosis. The effects of these TF on cardiomyocytes (CM) and their capacity to be taken up by the cell are unknown. The purpose of this study was to determine if direct supplementation of TF to the media bathing CM would increase the uptake into the cell, or alter the lipid profile. A time and dose dependent analysis of the fatty acid profile using gas chromatography was undertaken after Isolated CM were exposed to $50 \mu \mathrm{M}$ or $400 \mu \mathrm{M}$ of EA or VA for $6 \mathrm{~h}, 12 \mathrm{~h}$ or $24 \mathrm{~h}$. A dose dependent uptake of both VA and EA by the CM was observed after $24 \mathrm{~h}$. The mechanism for the uptake of the TF was examined through changes in expression levels of fatty acid transport proteins FATP1, FATP4 and CD36. However, after $24 \mathrm{~h}$ and $48 \mathrm{~h}$ of exposure to the TF, the expression levels were not significantly changed in any of these transport proteins. The mechanism responsible for the TF uptake into the CM remains unclear. Supported by CIHR, the Heart and Stroke Foundation of Canada, St Boniface Hospital Foundation

\section{6}

\section{ROLE OF THE SODIUM/CALCIUM EXCHANGER (NCX) IN CARDIAC VENTRICULAR REPOLARIZATION ASSESSED BY A NOVEL HIGHLY SELECTIVE NCX INHIBITOR}

\section{A Varró}

University of Szeged, Department of Pharmacology, Szeged, Hungary

The NCX is an important transport system in the heart and it is a major contributor to the maintenance of intracellular $\mathrm{Ca}^{2+}$ homeostasis. Although the sodium-calcium exchanger (NCX) is an electrogenic iontransport system potentially carrying net outward and/or inward currents, its contribution to the cardiac action potential have not yet been directly studied due to the lack of specific inhibitors. This is the first study that investigated the effects of selective NCX inhibition and compared to those of computer simulations by applying the conventional microelectrode and whole cell configuration of the patch-clamp techniques. ORM-10962, the highly selective NCX inhibitor blocked forward and reverse mode of NCX in dog ventricular myocytes with estimated EC50 values of $54 \mathrm{nM}$, and $68 \mathrm{nM}$, respectively without altering other important transmembrane ionic currents. Inhibition of NCX by ORM-10962 elicited modest and variable changes on action potential waveforms on different types of cardiac tissue preparations studied. The experimental results only partially agreed with in silico modelling results suggesting that our current understanding of NCX function is limited and further investigations are needed to clarify its pathophysiological role in arrhythmia initiation.

\section{7}

\section{ACTIVATION OF CARDIOPROTECTIVE SIGNALING IN RESPONSE TO IRRADIATION INJURY DEMONSTRATED IN RATS}

C Viczenczova ${ }^{1}$, B Szeiffova-Bacova ${ }^{1}$, B Kura ${ }^{1}$, Ch Yin $^{2}$, RC Kukreja ${ }^{2}$, J Slezak ${ }^{1}$, N Tribulova ${ }^{1}$

${ }^{1}$ Institute for Heart Research, Bratislava, Slovakia; ${ }^{2}$ Virginia Commonwealth University, Richmond, Virginia, USA

It has been established that impairment of direct cell-to-cell communication via connexin $(\mathrm{Cx})$ channels is implicated in heart dysfunction as well as tumor promotion. Of note, radiotherapy can reverse the down-regulation of $\mathrm{Cx}$ and inhibits cancer. We hypothesized that single irradiation injury may trigger cardiac defense mechanisms. To address this issue we investigated myocardial $\mathrm{Cx} 43 \mathrm{mRNA}$ and protein expression as well as PKC signaling in adult, male Wistar rats six weeks after exposure to $25 \mathrm{~Gy}$ irradion of the thorax. Total $\mathrm{Cx}-43$ protein and its functional phosphorylated forms were significantly increased in left ventricle of rats exposed to irradiation. In parallel, the level of miR-1 that down-regulates Cx43, was suppressed and expression of PKC-epsilon that phosphorylates $\mathrm{Cx}-43$, was increased in those animals. Irradiation also induced increase in PKC-delta and miR-21 expression suggesting activation of pro-hypertrophic and matrix metalloproteinase pathways. In conclusion, single irradiation of thorax triggers endogenous defense mechanisms that may be important in preservation heart function.

Funding: Provided by APVV 0241/11 and VEGA 0046/12. 


\section{8}

\section{RENAL DENERVATION IN HEART FAILURE: A NEW THERAPEUTIC PARADIGM}

\section{P Dhakal, K Liu, D Villarreal}

State University of New York Upstate Medical University, Syracuse, New York, USA

In heart failure, multiple extrinsic neurohumoral and intrinsic renal mechanisms interact to produce adjustments of renal hemodynamic and excretory function. Through its diverse actions on the kidney, enhanced renal sympathetic nerve activity promotes marked salt and water retention during the course of cardiac decompensation. Research in animal models by our group and others has demonstrated that renal nerve ablation produces significant natriuresis and diuresis in heart failure via enhancement of renal hemodynamics, as well as, the restoration of endogenous and exogenous natriuretic peptides> expression on the kidneys. With the recent advent of minimally invasive catheter-based technique of bilateral renal denervation in humans, clinical trials are addressing the safety of this procedure in systolic heart failure. Information derived from these initial studies expectedly will lead to more definitive research to determine the important therapeutic value of renal denervation in human heart failure. Funding: Supported by NIH, VAH and AHA.

\section{9}

\section{MICRORNAS IN CARDIOVASCULAR DEVELOPMENT, FUNCTION AND DISEASE}

\section{DZ Wang}

Department of Cardiology, Boston Children's Hospital,

Harvard Medical School, Boston, Massachusetts, USA

Congenital heart disease (CHD) represents the most common classes of birth defects in humans. Heart disease is the number one killer on both sides of the age spectrum, resulting in significant mortality and morbidity in children and adults. Despite intensive investigation, the genetic cause and the underlying molecular mechanism for $\mathrm{CHD}$ and other cardiovascular diseases remain poorly defined. miRNAs are a novel class of $\sim 22 \mathrm{nt}$ noncoding RNAs that regulate gene expression post-transcriptionally. Recent studies demonstrate that miRNAs play a central role in many biological processes and human diseases, including cardiovascular diseases. We show that loss-of-miRNAs in the cardiovascular system leads to severe defects in multiple cardiovascular structures in mice. We have generated multiple lines of knockout and transgenic mice for miRNAs. Many of these mice are viable and display a variety of cardiovascular defects. We will systematically analyze the phenotype of these mice. The ultimate goal of our research is to delineate the molecular pathways for the development and function of cardiovascular system and to use this information to design pharmacologic and genetic therapies for human CHD and other cardiovascular diseases, cardiac hypertrophy and heart failure.

\section{0}

CLINICAL FACTORS INFLUENCING THE DECISION TO INITIATE ANTICOAGULATION FROM A NOVEL PHYSICIAN ALERT SYSTEM FOR ATRIAL FIBRILLATION DETECTED ON CARDIAC IMPLANTED ELECTRONIC DEVICES

AW Wassef, B Hiebert, C Seifer

Division of Cardiology, University of Manitoba, Winnipeg,

Manitoba

BACKGROUND: Subclinical atrial fibrillation (AF) detected on Cardiac Implanted Electronic Devices (CIED) associates with increased stroke risk. We assessed the factors influencing the decision to initiate anticoagulation in a physician alert system.

METHODS: A system alerting referring physicians to the presence of AF detected on their patient's CIED was implemented at St Boniface Hospital, Winnipeg. We reviewed the Manitoba Drug Program Information Network (DPIN) and the hospital chart to assess if clinical scores factored into the decision making process for anticoagulation.

RESULTS: For patients with a CHADS2 $>1$, there was no difference for those anticoagulated vs those not in the CHADS2 score $(2.81 \pm 0.95$ vs $2.54 \pm 1.21$; $\mathrm{P}=0.20)$, CHADS2-VaSc score $(4.18 \pm 1.21$ vs $3.94 \pm 0.83 ; \mathrm{P}=0.23)$ or HASBLED score $(2.19 \pm 0.79$ vs $2.39 \pm 0.77 ; \mathrm{P}=0.07)$. On multivariate analysis, only being on
ASA (OR $0.20(0.08$ to 0.46$) ; \mathrm{P}<0.001)$ and having atrial fibrillation $>4 \mathrm{~h}(\mathrm{OR}$ 2.85 (1.07 to 7.56); $\mathrm{P}=0.035$ ) were predictive of anticoagulation.

CONCLUSIONS: Clinical risk factors predicting stroke and bleeding did not predict initiation of anticoagulation in a physician alert system for AF. Ongoing education is required for physicians regarding anticoagulation in AF.

\section{1}

\section{CELL-BASED THERAPY FOR THE FAILING HEART IN} HYPERTENSIVE HEART DISEASE (HHD)

\section{KT Weber}

Division of Cardiovascular Diseases, University of Tennessee Health Sciences Center, Memphis, Tennessee, USA

HHD is a major etiologic factor contributing to heart failure with preserved ejection fraction. Its morphologic features: widely scattered foci of microscopic scars, each representing a footprint of cardiomyocyte necrosis; and heterogeneity in cardiomyocyte size. Hypertrophied cells appear in response to arterial hypertension. Small cells of the myofiber syncytium are found bordering on microdomains of fibrosis, where they are ensnared by fibrillar collagen and subject to disuse. Features of these atrophic cells: re-expression of fetal genes (eg, b-myosin heavy chain); cKit stem cell antigen negative and without replication (BrdU labeling); express nitric oxide and reactive oxygen species; and activated redox-sensitive ligases (MuRF1, atrogin-1) of the ubiquitin proteasome system account for protein degradation. Myofibroblasts (myoFb) reside in close proximity and whose paracrine secretome may be responsible for myocyte dedifferentiation and size. Using a $\mathrm{Zn}^{2+}$-based antioxidant regimen: small myocytes and their prooxidant phenotype were rescued together with redifferentiation; myoFb disappeared; and improvements in diastolic and systolic ventricular dysfunction. Thus, atrophied myocytes represent an endogenous pool of viable cells which can be rescued to recover functional myocardium.

\section{2}

\section{HYPOTHERMIC INITIAL REPERFUSION IMPAIRS THE} FUNCTIONAL RECOVERY OF DCD HEARTS

CW White, A Müller, E Ambrose, L Hryshko, DH Freed Institute of Cardiovascular Sciences, University of Manitoba, Winnipeg, Manitoba

BACKGROUND: Donor hearts following circulatory death (DCD) have been proposed as an alternative source of organs for transplant; however, they experience significant ischemia prior to organ procurement. Initial reperfusion (IR) with an oxygenated cardioplegia may facilitate restoration of calcium homeostasis prior to myocardial contraction; however, these reparative processes may be inhibited under hypothermic conditions. We investigated the impact of IR temperature on the functional recovery of DCD hearts. METHODS: Pigs were anesthetized, mechanical ventilation was ceased, and cardiac arrest ensued. A 15-minute standoff period was observed and then hearts were reperfused with an adenosine-lidocaine cardioplegia at 3 different temperatures $\left(5^{\circ} \mathrm{C} ; n=6,25^{\circ} \mathrm{C} ; n=5\right.$, and $\left.35^{\circ} \mathrm{C} ; n=7\right)$. Hearts were then perfused ex vivo and myocardial function was assessed in a working mode.

RESULTS: Greater preservation of endothelial cell integrity (electron microscopy endothelial injury score: $5^{\circ} \mathrm{C}=3.2 \pm 0.5, \quad 25^{\circ} \mathrm{C}=1.8 \pm 0.2$, $35^{\circ} \mathrm{C}=1.7 \pm 0.3 ; \mathrm{P}=0.01$ ) and less myocardial injury (troponin I: $5^{\circ} \mathrm{C}=91 \pm 6$, $25^{\circ} \mathrm{C}=64 \pm 16,35^{\circ} \mathrm{C}=57 \pm 7 \mathrm{pg} / \mathrm{mL} ; \mathrm{P}=0.04$ ) were evident in hearts reperfused at warmer temperatures. IR under hypothermic conditions impaired the recovery of indexed cardiac output $\left(5^{\circ} \mathrm{C}=3.9 \pm 0.8,25^{\circ} \mathrm{C}=6.2 \pm 0.4\right.$, $\left.35^{\circ} \mathrm{C}=6.5 \pm 0.0 .6 \mathrm{~mL} / \mathrm{min} / \mathrm{gram} ; \mathrm{P}<0.01\right)$.

CONCLUSIONS: Avoidance of profound hypothermia during IR with an adenosine-lidocaine cardioplegia minimizes injury and improves the functional recovery of DCD hearts.

\section{3}

\section{THE ROLE OF GENDER IN MYOCARDIAL RESPONSES TO STRESS}

\section{Wittnich}

Department of Surgery, University of Toronto, Toronto, Ontario

Although conflicting reports exist on whether there are sex-related differences in the incidence and outcomes of cardiovascular disease, those that do suggest a role for steroid hormones since their receptors have been 
documented in the heart. Whether this sex bias exists in the young is also controversial with some evidence suggesting young females are at greater disadvantage than their male counterparts during stress. Specifically, animal studies have shown that when exposed to ischemia, hearts of newborn females have more dramatic reductions in energy levels and develop more significant acidosis than males. Also, evidence exists that adult females have higher heart rates, cardiac output and stroke volume when compared to males. However, when isolated muscle preparations were used, function was actually lower in females versus males. Interestingly, hormone specific studies show that testosterone administration to adults appeared to increase cardiac performance, while others showed that it increased the stiffness of the ventricle due to increased collagen synthesis, thereby reducing diastolic performance. The reasons for these seemingly conflicting results may reflect effects of sex hormones that are dependent on the conditions being studied. Evidence based findings will be reviewed to clarify the role of gender in myocardial responses to stress.

\section{4}

EFFECTS OF STORE-OPERATED $\mathrm{CA}^{2+}$-CHANNEL

BLOCKERS ON BLOOD PRESSURE AND VASCULAR SMOOTH MUSCLE CELLS

\section{YJ Xu, V Elimban, NS Dhalla}

Institute of Cardiovascular Sciences, St Boniface Hospital Research Centre, University of Manitoba, Winnipeg, Manitoba

L-type $\mathrm{Ca}^{2+}$-antagonists have been used clinically as anti-hypertensive agents; however, the effects of store-operated $\mathrm{Ca}^{2+}$ blockers such as SKF36365 (SK) or gandolinium (Gd) on blood pressure are not known. In this study, we found that both SK and Gd reduced systolic and diastolic blood pressures in rats in a dose and time dependent manner. While both agents had no effect on basal $\left[\mathrm{Ca}^{2+}\right] \mathrm{i}$, the increase in $\left[\mathrm{Ca}^{2+}\right]$ i due to lysophosphatidic acid (LPA) was depressed, unlike the norepinephrine-induced increase in $\left[\mathrm{Ca}^{2+}\right] \mathrm{i}$ in aortic smooth muscle cells. The angiotensin II-induced increase in $\left[\mathrm{Ca}^{2+}\right]$ i was also attenuated by both SK and Gd whereas Gd, unlike SK, did not alter the endothelin-induced increase in $\left[\mathrm{Ca}^{2+}\right]$ i. Cell proliferation, as determined by cell number and thymidine incorporation in the absence or presence of LPA, was reduced by SK, whereas Gd had no effect on this parameter. Verapamil-induced reduction in diastolic blood pressure and $\left[\mathrm{Ca}^{2+}\right]$ i were potentiated by SK. The results suggest that SK or Gd may be used alone or combination with L-type $\mathrm{Ca}^{2+}$, antagonists for the treatment of hypertension.

\section{5}

\section{HYDROGEN SULFIDE SIGNALING IN REGULATION OF CARDIOVASCULAR FUNCTIONS}

\section{G Yang}

Cardiovascular and Metabolic Research Unit, Lakehead University, Thunder Bay, Ontario

The physiological and pathological roles of hydrogen sulfide $\left(\mathrm{H}_{2} \mathrm{~S}\right)$ in the regulation of cardiovacular functions have been recognized. Deficiency of CSE in mice leads to decreased endogenous $\mathrm{H}_{2} \mathrm{~S}$ level, age-dependent increase in blood pressure, and impaired endothelium-dependent vasorelaxation. Decreased endogenous $\mathrm{H}_{2} \mathrm{~S}$ production also predisposes mice to vascular remodeling and increased neointima formation and early development of atherosclerosis. Exogenous applied $\mathrm{H}_{2} \mathrm{~S}$ protects vascular tissues from atherogenic damage. SMCs and aortic explants isolated from CSE knockout mice exhibited more proliferation, migration and outgrowth compared with that from wild-type mice. CSE deficiency elevates resting-membranepotential of SMCs and eliminated methacholine-induced endotheliumdependent relaxation of mesenteric arteries, but not that of aorta. $\mathrm{H}_{2} \mathrm{~S}$ also stimulates eNOS S-sulfhydration and eNOS dimer stability in endothelial cells, resulting in increased $\mathrm{NO}$ bioavailability. Taken together, $\mathrm{H}_{2} \mathrm{~S}$ acts as an endothelium-derived hyperpolarizing factor and a regulator for SMC phenotypic switch, and $\mathrm{CSE} / \mathrm{H}_{2} \mathrm{~S}$ system may be a novel target for the treatment of vascular occlusive disorders.

Acknowledgements: Grant funding provided by CIHR and HSFC to GY.

\section{6}

\section{CALCIUM AND STRESS MODULATE SERCA2 AND CASQ2 GENE TRANSCRIPTION}

A Zarain-Herzberg, J Fragoso-Medina, R Estrada-Avilés

Biochemistry Department, School of Medicine, National

Autonomous University of México, México City, Mexico

SERCA2a and calsequestrin (CASQ2) participate in the control of cytoplasmic $\mathrm{Ca}^{2+}$ concentration $\left(\left[\mathrm{Ca}^{2+}\right] \mathrm{c}\right)$. Thapsigargin $(\mathrm{Tg})$ inhibits SERCA2a resulting in an increased $\left[\mathrm{Ca}^{2+}\right]_{\mathrm{c}}$ and induces endoplasmic reticulum stress (ERS) by emptying $\mathrm{Ca}^{2+}$ from the sarcoplasmic reticulum. Tg increased SERCA2a mRNA and transcription of the human SERCA2 promoter in neonatal rat cardiomyocytes. Cyclosporine A (CsA) prevented Tg induced SERCA2a mRNA and transcription of hSERCA2 promoter constructs. Mutation of a consensus NFAT element in the hSERCA2 promoter blunted transcriptional increase in response to Tg treatment and transactivation by NFATc3 overexpression. Mutation of a hSERCA2 promoter CCAAT-box and a YY1 element that are part of an ERS response element (ERSE) did not respond to Tg, demonstrating that a proximal NFAT responsive site and a fully functional ERSE of the hSERCA2 gene are necessary for its regulation by $\left[\mathrm{Ca}^{2+}\right]_{\mathrm{c}}$ and in response to ERS. Inhibition of NFAT activation with CsA or INCA- 6 reduced transcription of hCASQ2 promoter constructs but NFATc3 overexpression increased it. Mutagenesis of a proximal hCASQ2 promoter MEF2 element abolished activation by NFATc 3 overexpression, suggesting that MEF2 interacts with NFATc3 regulating CASQ2 transcription.

\section{7}

\section{PROTEOLYTIC DAMAGE OF HERG AND CARDIAC ARRHYTHMIAS}

\section{S Zhang}

Department of Biomedical and Molecular Sciences, Queen's University, Kingston, Ontario

The human ether-a-go-go-related gene ( $h E R G)$ encodes the pore-forming subunit of the rapidly activating delayed rectifier potassium channel $\left(\mathrm{I}_{\mathrm{Kr}}\right)$, which is important for cardiac repolarization. hERG channel dysfunction causes long QT syndrome, predisposing affected individuals to fatal ventricular arrhythmias and sudden death. Here, we demonstrate that among various ion channels expressed in HEK cells and in isolated cardiomyocytes, the serine protease proteinase $\mathrm{K}$ selectively cleaved the $\mathrm{hERG} / \mathrm{I}_{\mathrm{Kr}}$ channel. Replacement of the hERG S5-pore linker with the EAG S5-pore linker (hERG-EAGS5P) completely eliminated hERG's proteinase K sensitivity. In addition, the scorpion toxin $\mathrm{BeKm}-1$, that selectively binds to the S5-pore linker of hERG channels, protects hERG from proteinase $\mathrm{K}$-mediated damage. The reduction in mature ERG expression was also observed in a rabbit cardiac ischemia model and in hERG-HEK cells cultured in hypoxic conditions. BeKm-1 effectively prevented the hypoxiainduced reduction of the mature hERG expression. Thus, the S5-pore linker of hERG represents a unique target site for protease-induced cleavage, and protecting hERG channels from proteolytic damage using peptides may represent a novel strategy to prevent and treat cardiac arrhythmias associated with ischemic heart disease.

Acknowledgements: Grant funding provided by CIHR to SZ.

\section{8}

\section{HEART FAILURE THERAPIES: NEW TARGETS AND NEW FRONTIERS}

\section{S Zieroth}

Department of Medicine, Section of Cardiology, University of Manitoba, Winnipeg, Manitoba

Despite advances in medical therapy, heart failure continues to represent a significant clinical and financial burden in the healthcare domain. New approaches are needed and therapeutic targets are being expanded to include novel agents such as relaxin and myosin activators. Functional foods and 'nutraceuticals' such as resveratrol and other translational research explorations like gene therapy attempt to tackle the heart failure epidemic. An update in the advances in heart failure therapies will be presented. 


\section{A}

Abdelmoneim SS.................001

Abrenica B. . . . . . . . . . . . . . . . . . . 046

Abu-El Rub E. . . . . . . . . . . . . . . 003

Adameova A ................004, 102

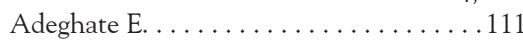

Agrawal D ...................... 005

Agrawal DK...................118

Ajithkumar GS . . . . . . . . . . . . . .063

Akolkar G .................002, 009

Aliani M ..................007, 038

Alomar F . . . . . . . . . . . . . . . . . . . . 014

Alonso F. . . . . . . . . . . . . . . . . . 011

Ambrose E ......................132

Ammar HI . . . . . . . . . . . . . . . . . .003

Ammar RI ......................003

Anand-Srivastava MB ..............006

Aroutiounova N. .................. 104

Austria J........................007

\section{B}

Baechler CJ .....................008

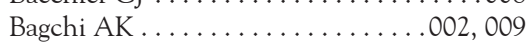

Bagchi R. . . . . . . . . . . . . . . . 027, 104

Bagchi RA ....................... 010

Bansilal S . . . . . . . . . . . . . . . 011

Barancik M. ..................... 013

Barta J......................... 012

Bartekova M. . . . . . . . . . . . . . . . 013, 069

Barudzic N . . . . . . . . . . . . . . . . . . 059

Baydoun AR. . . . . . . . . . . . . . . . . . 079

Berhanu T . . . . . . . . . . . . . . . . . . . 097

Bernatova I. . . . . . . . . . . . . . . . . . . . . .069

Bidasee K .......................111

Bidasee KR...................... 014

Binilraj SS . . . . . . . . . . . . . . . .063

Bkaily G......................015

Blackman AO . . . . . . . . . . . . . . . 016

Blackwood D . . . . . . . . . . . . . . . 007

Bordun KA...............001, 017, 049

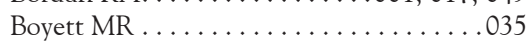

Brabcova I . . . . . . . . . . . . . . . . . .092

Brenes-Salazar JA. . . . . . . . . . . . . . . . 018

Buchholz B..................... 045

Burgon PG ......................019

Buttar H.........................020

\section{$\mathrm{C}$}

Caligiuri $S \ldots \ldots \ldots \ldots \ldots \ldots \ldots . \ldots 38$

Caligiuri SPB . . . . . . . . . . . . . . 021

Carnicka S............004, 022, 069, 102

Castaneda E...................... 023

Castellano J . . . . . . . . . . . . . . . . . 011

Cattin ME ...................... 019

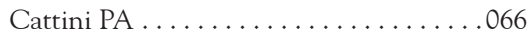

Cavasin M . . ....................117

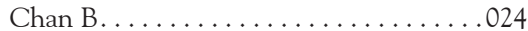

Chan D .......................101

Chaudhary R ...........021, 054, 101

Cheema SK . . . . . . . . . . . . .... 025

Chen S......................118

Chen-Scarabelli C.................. 106

Cheung D. .................... 049

Chugh H .................................... 115

Chugh S.....................121

Chugh SS....... 026, 088, 089, 096, 115

Cook T..........................................

Czubryt M ................027, 104

Czubryt MP .................. 010

$\mathrm{D}$

D'Souza A ................ 035, 111 da Silva F . . . . . . . . . . . . . . . . 123

da Silva M ..................... 017

Das S ......................... 028

Dawn B ........................ 029

de Castro Brás LE. . . . . . . . . . . . . . . 122

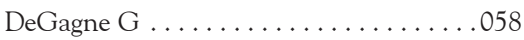

Del Core M . . . . . . . . . . . . . . . . . 005

Demos-Davies K. . . . . . . . . . . . . . . 117

Deniset JF. . . . . . . . . . . . . . . .056

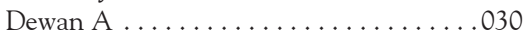

Dhakal P ....................... 128

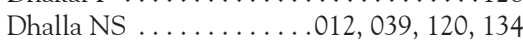

Dhingra R .................. 031

Dhingra S.............003, 032, 051

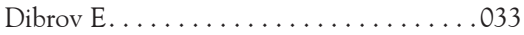

Ding H....................... . . . . . . . . . . . . .

Ding $\mathrm{Z} \ldots \ldots \ldots \ldots \ldots \ldots \ldots \ldots$

Dirksen RT. . . . . . . . . . . . . . . . . . . .094

Dixon IMC . . . . . . . . . . . . . . . . 058

Djuric D . ...................... 059

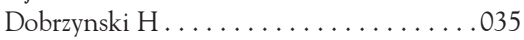

Dolinsky VW . . . . . . . . . . . . . . . .034

Donato M........................ 045

Dorn II G..................... 031

dos Reis FJ . . . . . . . . . . . . . . . . 072

Duarte JJ....................... 036

Duhamel TA ..................037

$\mathrm{E}$

Edel A ..................033, 038

Elimban V .................039, 134

Elliott J...................... 035

Elsayed HE . . . . . . . . . . . . . . . . . . . .003

Estrada-Avilés R. .................136

\section{$\mathrm{F}$}

Falck JR ...................... 091

Fandrich R..................... 049

Fandrich RR. . . . . . . . . . . . . . . . . . 066

Fanous Y..................... 040

Feldman RD...................041

Ferdinandy P . . . . . . . . . . . . . . 004

Ferguson B . . . . . . . . . . . . . . . . . 117

Ferko M . . . . . . . . . . . . . . . . . 022

Fliegel L . . . . . . . . . . . . . . . . . . . . . . .042

Fragoso-Medina J . . . . . . . . . . . . . . . 136

Freed DH . ......... 043, 075, 086, 132

Freeman A ..................... 011

Friesen C . . . . . . . . . . . . . . . 037

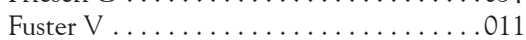

\section{G}

Gablovsky I . . . . . . . . . . . . . . . .022

Gabr HM . . . . . . . . . . . . . . . . . 003

Gaisler da Silva F. . . . . . . . . . . . . . . . . 040

Gandhi TR. . . . . . . . . . . . . . . . . . . . 044

Gang H. .................... 073

Ganguly R ............. 054, 077, 125

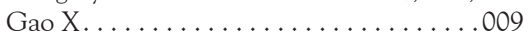

Gavazzoni M ......................106

Gelpi RJ ....................... . . . . . .

Gilchrist JSC . . . . . ............. 046

Giricz Z. . . . . . . . . . . . . . . . . . . . . . 004

Gomes OM. ...............016, 047

Gomes WJ ....................... 072

Goyal RK.....................048

Goyal V . . . . . . . . . . . . . . . . . . . . 049

Green M.......................001

Griecsova S . . . . . . . . . . . . . . . . 102

Grierson R.................... 050

Gros R ....................041

Gross GJ.................. 091
Grothey A .....................................

Guan Q . . . . . . . . . . . . . . . . . 051

Gunson K........ 088, 089, 096, 115, 121

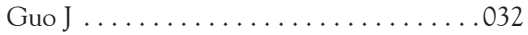

Gupta M..................... . . . . . .

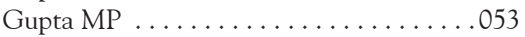

Gupta RC..................... 085

Guzman R ..........007, 033, 038, 099

\section{$\mathrm{H}$}

Hasanally D . . . . . . . . . . . . . . . 054, 101

Hayashi H ....................... . . . . . . . .

Hedley TE ...................... . 056

Herrmann J ...................... 017

Heydemann A . . . . . . . . . . . . . . . . . . 097

Hiebert B ................. 130, 086

Hlaváčková M . . . . . . . . . . . . . . . . .0056

Holey-Cuthrell J . . . . . . . . . . . . . . . . . 097

Howarth C. . . . . . . . . . . . . . . . . . 111

Howlett SE. . . . . . . . . . . . . . . . . 057

Hryshko L . . . . . . . . . . . . . . . . 132

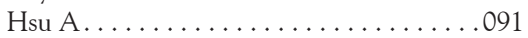

Huang R...................... 001

Huang XP. . . . . . . . . . . . . . 032

Huertas-Vazquez A. ............. 026, 096

Hughes B . . . . . . . . . . . . . . . . . . . .024

Hunter W........................ 005

\section{I}

Imig JD . . . . . . . . . . . . . . . . . . 091

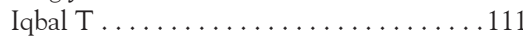

Ivanova M . . . . . . . . . . . . . . . . 013

$\mathrm{J}$

Jackowski G. . . . . . . . . . . . . . . 108 Jahan F...................... . . . . . . .

Jakovljevic V . . ............................ 059

Jassal D....................031, 066

Jassal DS. ........ 001, 002, 017 049, 101

Jones SP . . . . . . . . . . . . . . . . 060

Jugdutt B . . . . . . . . . . . . . . . 061

Jui J. . . . . . . . 088, 089, 096, 115, 121

\section{$\mathrm{K}$}

Kalous M ........................ 092

Kardami E. . . . . . . . . . . . . . . . . . . 049, 066

Karmazyn M...................062

Kartha CC . . . . . . . . . . . . . . . . . .063

Kayode B ....................... 025

Khan MAH . . . . . . . . . . . . . . . . . .091

Khanna V....................... 048

Khullar M....................064

Kim C. ............................ 089

Kirshenbaum LA ....... 031, 054, 073, 101

Kohli M ...................001,017

Kolar F.................. 065, 092

Koleini N . . . . . . . . . . . . . . . . . . . . . 066

Kovacs A ..................... 012

Kukreja RC ..............067, 127

Kumada Y...................... 055

Kumamoto H..................055

Kumar A.......................... . 064

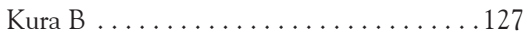

Kyselovic J ...................068

$\mathrm{L}$

La Vallée R. . . . . . . . . . . . . . . . . . 038

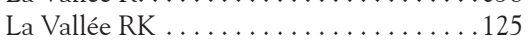

Ledvenyiova V............... 069, 102

Levick SP . . . . . . . . . . . . . . . . . . . 070

Li P........................ 032

Li RK ..............032, 071, 076 
Li SH ..................032,076

Li Y..................... 017, 086

Lima ML .....................072

Lin J ................... 031, 073

Lindsey ML ...................122

Liu K ........................ 128

Longenbach S ..................001

Lopaschuk GD . . . . . . . . . . . . . . . . . 074

Lougon LN...................... 072

Luc JGY . . . . . . . . . . . . . . . . 075

Ludke A.......................076

\section{$\mathrm{M}$}

Maan G .......................079

Maddaford T . . . . . . . . . . . . . . . . 038

Maddaford TG............033, 054, 077

Major J...................040, 123

Makino N. . . . . . . . . . . . . . . 055, 078

Mandal S ..................002, 049

Mandikova P . . . . . . . . . . . . . . . . . . 092

Margulets V . . . . . . . . . . . . . . . . . 101

Mariani R..............096, 115, 121

Marijon E...................... 088

Matsuo H.......................055

Mayhan WG ........................ 014

McKinsey TA...............080, 117

McNally E . . . . . . . . . . . . . . . . . . . . . 097

Mehta JL ....................... 081

Michelakis ED ....................075

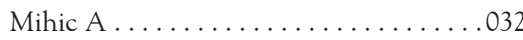

Miller JD . . . . . . . . . . . . . . . ...082

Mishra PK .....................083

Mittal A........................064

Mlynarova J . . . . . . . . . . . . . . . . 123

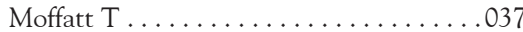

Mohyi PM .........................0. 085

Morad M ..................... 084

Mull A ..........................097

Muller A..................... 017

Müller A.........................132

Müller AL ......................086

Mulvagh S . . . . . . . . . . . . . . . . 017

Mulvagh SL . . . . . . . . . . . . . . . . 001

Muntean D..................... 087

Murarikova M ...............022, 102

\section{$\mathrm{N}$}

Nagendran J........................ 075

Narayanan K . . . . . . . . . . . . . . 088, 089

Narula J . . . . . . . . . . . . . . . . . . . 090, 106

Nashed MB . . . . . . . . . . . . . . . . . 003

Neckar J . . . . . . . . . . . . . . . . . . . . . 092

Neckár J . . . . . . . . . . . . . . . . . . . 091

Nhola LF . . . . . . . . . . . . . . . . . . . . .001

Ni H. . . . . . . . . . . . . . . . . . . . . .093

Nickel B...................... 049

Nickel BE. . . . . . . . . . . . . . . . . . . .066

Niraula S . . . . . . . . . . . . . . . . . . . 049

Novakova O. ....................092

\section{$\mathrm{O}$}

O'Hara K ......................037

Oceandy D . . . . . . . . . . . . . . . . . .035

Okruhlicova L . . . . . . . . . . . . . . 013

Ostadal B .......................095

O-Uchi J.........................094

Parks RJ .................. 057

Patel N........................ 048

Paulin R.....................0075

Pelham C.......................005

Peng Z . . . . . . . . . . . . . . . . . . . .051
Phlavan S. ......................084

Pierce G................007, 038

Pierce GN ......021, 033, 046, 054, 056,

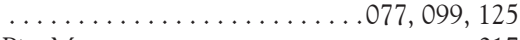

Pitz M.......................017

Pontes JCDV . . . . . . . . . . . . . . . . . 036

Pravenec M . . . . . . . . . . . . . . . . . . 092

Premecz S. . ............017, 049, 101

\section{$\mathbf{R}$}

Raddino R . . . . . . . . . . . . . . . . . 106

Radwan M . . . . . . . . . . . . . . . . . .005

Rajtik T .......................004

Ramila KC......................... 107

Ramos M ........................ 040

Ramos MSC. . . . . . . . . . . . . . . . . . 123

Rashidkhani B ................046

Rattan S................058, 064

Ravandi A ............021, 054, 101

Ravingerova T . ......004, 022, 069, 102

Reinier K ..... .026, 088, 089, 096, 115, 121

Rizzuto R . . . . . . . . . . . . ........094

Roberts N. . . . . . . . . . . . . . . . . . . . . . 097

Roberts R........................ 103

Roche P . . . . . . . . . . . . . . . . 027, 104

Rodrigues B . . . . . . . . . . . . . . . . .098

Rodriguez M. . . . . . . . . . . . . . . . . . 045

Rodriguez-Leyva D. ..... .033, 038, 099, 100

Rodriguez-Portelles A . . . . . . .099, 100, 033

\section{$\mathrm{S}$}

Sabbah HN ................ 085, 105

Sahni G . . . . . . . . . . . . . . . . . . . . 106

Salih M .............030, 040, 123

Sandhu N. . . . . . . . . . . . . . . . . . . 017

Sanganalmath SK ................. 012

Sanjay G. . . . . . . . . . . . . . . . . . .063

Sankaralingam S . . . . . . . . . . . . . 074

Santiago JJ ......................066

Sanz G . . . . . . . . . . . . . . . . . . . . . 011

Scarabelli TM ...................106

Schaffer SW.....................107

Schulz R.......................024

Schweitzer R .................... 010

Seifer C . . . . . . . . . . . . . . . . . . 130

Shaikh NA. . . . . . . . . . . . . . . . . . . 108

Sharma V....................... 109

Sheikh F........................ 110

Sheu SS ......................... . 094

Silhavy J. . . . . . . . . . . . . . . . . . . . . . . .092

Simoncikova P.................. 013

Singal PK........... . 002, 009, 017, 049

Singh J . . . . . . . . . . . . . . . . . . 014, 111

Singh M..................... 018

Singla D . . . . . . . . . . . . . . . . . 112

Slezak J. ...................113, 127

Smith GL. . . . . . . . . . . . . . . . . . . . . . 094

Sobral J........................ 016

Sonoda MT . . . . . . . . . . . . . . . . . . . .040

Spettell C......................011

Spilotoir J. . . . . . . . . . . . . . . . . . . . 117

Srisakuldee W . . . . . . . . ..........066

Srivastava AK .................... 114

Stammers A . . . . . . . . . . . . . . . . . 037

Stecker E ..................... 096

Stecker EC . . . . . . . . . . . . . . . . . . . 115

Steenbergen C . . . . . . . . . . . . . . 028

Steinberg G . . . . . . . . . . . . . . . . .011

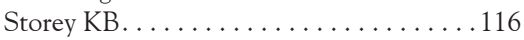

Stratton M.....................117

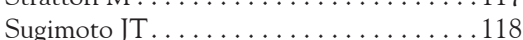

Sur S........................ 118

Susser SE . . . . . . . . . . . . . . . . . 037
Svec P .........................004

Swier V .......................005

Szeiffova-Bacova B. . . . . . . . . . . . . . 127

Szobi A.......................004

$\mathrm{T}$

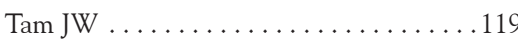

Tang L .......................... . . . .

Tappia PS .......................120

Teixeira FOG. .................. 072

Teodorescu C. . .026, 088, 089, 096, 115, 121

Thakur S . . . . . . . . . . . . . . . . . . 079

Thliveris J. . . . . . . . . . . . . . . . 017

Tian Y............................ 122

Trentin Sonoda M . . . . . . . . . . . . . . . . 123

Tribulova N . ....................... 127

Triggle C..................... 030

Tuana B . . . . . . . . . . . . . . . . . .0030

Tuana BS .................040, 123

Turan B ....................... 124

\section{$\mathrm{U}$}

Uy-Evanado A . . . . 026, 089, 096, 115, 121

Uy-Evanadoy A . . . . . . . . . . . . . . .088

\section{$\mathrm{V}$}

Varró A .......................126

Viczenczova C . . . . . . . . . . . . . . . . 127

Villaraga H. . . . . . . . . . . . . . . . . .017

Villarraga HR.......................001

Villarreal D. . . . . . . . . . . . . . . . . . 128

Vinado E . . . . . . . . . . . . . . . . . . . . 011

Vinitha A....................063

Wall D ...................... 051

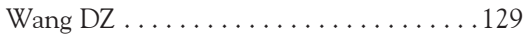

Wang W. . . . . . . . . . . . . . . . . . . . . . . . 094

Wang X ....................... 081

Wassef AW........................ 130

Weber KT. . . . . . . . . . . . . . . . 131

Wei H. . . . . . . . . . . . . . . . . . . .011

Weighell W ...................... 038

Weisel RD . . . . . . . . . . . . . . . 032,076

Weissova R. . . . . . . . . . . . . . . . . . 092

White C....................... 017

White CW ........................ 132

Wigle JT. ....................058

Wittnich C. . . . . . . . . . . . . . . . 133

Wu J................. 032, 076

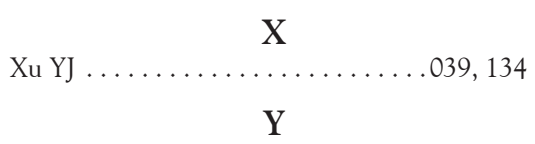

Yang B ...................... 051

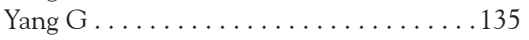

Yang X . . . . . . . . . . . . . . . . . . . . . . .009

Yin $\mathrm{Ch} \ldots \ldots \ldots \ldots \ldots \ldots \ldots \ldots \ldots$

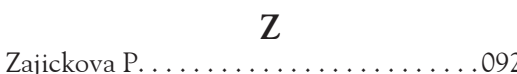

Zalesak M.......................... 102

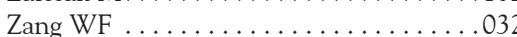

Zarain-Herzberg A . . . . . . . . . . . . 136

Zhang L . . . . . . . . . . . . . . . . . . . . . . . 089

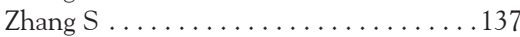

Zhang XH ....................084

Zhao JY .........................075

Zieroth S ......................138

Zivkovic V.....................059

Zurmanova J....................092 
NOTES 